\title{
A construction of lower-bounded generalized twisted modules for a grading-restricted vertex (super)algebra
}

\author{
Yi-Zhi Huang
}

\begin{abstract}
We give a general, direct and explicit construction of lower-bounded generalized twisted modules satisfying a universal property for a grading-restricted vertex (super)algebra $V$ associated to an automorphism $g$ of $V$. In particular, when $g$ is the identity, we obtain lower-bounded generalized $V$-modules satisfying a universal property. Let $W$ be a lower-bounded graded vector space equipped with a set of "generating twisted fields" and a set of "generator twist fields" satisfying a weak commutativity for generating twisted fields, a generalized weak commutativity for one generating twisted field and one generator twist field and some other properties that are relatively easy to verify. We first prove the convergence and commutativity of products of an arbitrary number of generating twisted fields, one twist generator field and an arbitrary number of generating fields for $V$. Then using the convergence and commutativity, we define a twisted vertex operator map for $W$ and prove that $W$ equipped with this twisted vertex operator map is a lower-bounded generalized $g$-twisted $V$-module. Using this result, we give an explicit construction of lower-bounded generalized $g$-twisted $V$-modules satisfying a universal property starting from vector spaces graded by weights, $\mathbb{Z}_{2}$-fermion numbers and $g$-weights (eigenvalues of $g$ ) and real numbers corresponding to the lower bounds of the weights of the modules to be constructed. In particular, every lowerbounded generalized $g$-twisted $V$-module (every lower-bounded generalized $V$-module when $g$ is the identity) is a quotient of such a universal lower-bounded generalized $g$-twisted $V$-module (a universal lower-bounded generalized $V$-module).
\end{abstract}

\section{Introduction}

In the representation theory of associative algebras and Lie algebras, modules satisfying universal properties (for examples, free modules, Verma modules and so on) in suitable categories of modules play a fundamental role. Modules in these categories are quotients of these biggest or universal modules and therefore can be studied using these modules whose structures are relatively simple to understand.

In the representation theory of vertex (operator) (super)algebras and conformal field theory, finding a construction of modules satisfying universal properties in suitable categories 
of modules has been a long-standing problem. Finding such a construction will provide us with a powerful tool and will allow us to use the powerful homological algebra techniques (for example, the construction and applications of resolutions of modules) for the study of modules for vertex (operator) (super)algebras. To study the fixed-point subalgebra of a vertex (operator) (super)algebra under a group of automorphisms, we have to construct and study twisted modules. It is also a long-standing problem in the case that the automorphism is of finite order to find such a construction of twisted modules satisfying universal properties in suitable categories of twisted modules.

In this paper, we give a general, direct and explicit construction of lower-bounded generalized twisted modules satisfying a universal property for a grading-restricted vertex (super)algebra $V$ associated to an automorphism $g$ of $V$. In particular, in the case that $g$ is the identity, our construction give a general, direct and explicit construction of lower-bounded generalized $V$-modules. Our construction is for an arbitrary automorphism of the algebra. In particular, in the case that the automorphism of the algebra is of infinite order and does not act on the algebra semisimply, our construction gives lower-bounded generalized twisted modules whose twisted vertex operators in general involve logarithm of the variable.

Twisted modules associated to automorphisms of finite order of a vertex operator algebra were introduced by Frenkel, Lepowsky and Meurman in their construction [FLM1] [FLM2] [FLM3 of the moonshine module vertex operator algebra $V^{\natural}$. Twisted module associated a general automorphism $g$ of a vertex operator algebra $V$ were introduced by the author in [H1]. One of the main conjecture in the representation theory of vertex operator algebras is that for a suitable vertex operator algebra $V$ and a finite group $G$ of automorphisms of $V$, the category of $g$-twisted $V$-modules for all $g \in G$ has a natural structure of $G$-crossed braided tensor category satisfying additional properties (see [H3]). This conjecture follows from another stronger conjecture stating that twisted intertwining operators (see [H5]) among $g$-twisted $V$-modules for $g \in G$ satisfy associativity, commutativity and modular invariance property (see also [H3]). The second conjecture corresponds to a construction of orbifold conformal field theories and its solution will certainly depend on a deep understanding of twisted $V$-modules.

Twisted modules for vertex (operator) (supper)algebras have been constructed and studied in many papers (see for example, [Le1], [FLM2], [Le2], [FLM3], [D], [DL], DonLM1, [DonLM2], [Li], [BDM], DoyLM1, DoyLM2, [BHL], [H1], [B], [Y], and the references in these papers). But these constructions and studies are for special classes of vertex operator algebras and/or special classes of automorphisms. To study twisted modules, twisted intertwining operators and the category of twisted modules, we need a general construction of twisted modules. In principle, twisted modules can be constructed using the the functors constructed in [HY] from categories of modules for the associative algebras introduced in [DonLM1] (for automorphisms of $V$ of finite orders) and in [HY] (for general automorphisms) to suitable categories of twisted modules. But this indirect approach is very difficult to use in general because the abstract functors in [HY] from the categories of modules for the associative algebras to the categories of suitable twisted modules are not equivalence of categories. It is therefore important to have a general, direct and explicit construction of 
suitable twisted modules satisfying universal properties. As we mentioned above, finding such a construction is a long-standing problem even in the case that the automorphism is of finite order or is even the identity. We solve this problem in this paper in the category of lower-bounded generalized $g$-twisted $V$-module for a general automorphism $g$ of a general grading-restricted vertex (super)algebra $V$.

The approach used in our construction is the one that the author developed for the first construction of grading-restricted vertex algebras in [H2]. But the construction of lowerbounded generalized twisted modules in this paper, especially of those twisted modules whose twisted vertex operators involving the logarithm of the variable, is much more difficult than the one in [H2], because the twisted vertex operators are multivalued and because we do not have skew-symmetry for twisted modules (even for modules). Besides twisted vertex operators, one crucial ingredient in the construction in this paper is the twist vertex operators introduced and studied in [H6].

Our construction is divided into two steps. We first prove a general construction theorem which will be very useful also for the constructions of grading-restricted twisted modules, twisted modules and other types of lower-bounded generalized twisted modules. Let $W$ be a lower-bounded graded vector space equipped with a set of "generating twisted fields" and a set of "generator twist fields" satisfying a weak commutativity for generating twisted fields, a generalized weak commutativity for one generating twisted field and one generator twist field and some other properties that are relatively easy to verify. We first prove the convergence and commutativity of products of an arbitrary number of generating twisted fields, one twist generator field and an arbitrary number of generating fields for $V$. Then using the convergence and commutativity, we define a twisted vertex operator map for $W$ and prove the construction theorem that $W$ equipped with this twisted vertex operator map is a lower-bounded generalized $g$-twisted $V$-module. If $W$ is grading-restricted, we obtain a grading-restricted twisted module and if in addition the operator $L_{W}(0)$ acts on $W$ semisimply, we obtain a twisted module. In the special case that $g=1_{V}$, we obtain lower-bounded generalized modules, grading-restricted generalized modules and modules.

Then using this construction theorem, we give an explicit construction of a lower-bounded generalized $g$-twisted $V$-module $\widehat{M}_{B}^{[g]}$ satisfying a universal property starting from a vector space $M$ graded by weights, $\mathbb{Z}_{2}$-fermion numbers and $g$-weights (eigenvalues of $g$ ) and a real number $B$ less than the real parts of all weights of homogeneous elements of $M$. The real number $B$ is in fact a lower bound of the weights of $\widehat{M}_{B}^{[g]}$ and, roughly speaking, $M$ together with the algebra $V$ gives the generators of $\widehat{M}_{B}^{[g]}$. In particular, every lower-bounded generalized $g$-twisted $V$-module (every lower-bounded generalized $V$-module when $g$ is the identity) is a quotient of such a universal lower-bounded generalized $g$-twisted $V$-module (a universal lower-bounded generalized $V$-module).

The construction and results obtained in this paper can be used to study a number of problems in the representation theory of vertex (operaor) (super)algebras. We shall discuss these apllications in future papers. We shall also construct and study examples of twisted modules for lattice, affine Lie and Virasoro vertex operator algebras in future papers using the construction and results in this paper. 
The formulations and construction in the present paper are based on the formulations and results in [H6]. We refer the reader to [H6] for the basic definitions of grading-restricted vertex (super)algebra, generalized twisted modules and variants and twist vertex operator, conventions on formal and complex variables, and results on twist vertex operators and their proofs.

This paper is organized as follows: In Section 2, assuming that $V$ is generated by a set of fields $\left\{\phi^{i}(x)\right\}_{i \in I}$ and $g$ is an automorphism of $V$, we introduce our data, a graded vector space $W$ with an action of $g$, a set $\left\{\phi_{W}^{i}(x)\right\}_{i \in I}$ of generating twisted fields and a set $\left\{\psi_{W}^{a}(x)\right\}_{a \in A}$ of generator twist fields and two operators $L_{W}(0)$ and $L_{W}(-1)$, and our assumptions on these data, including, in particular, a weak commutativity for generating twisted fields and a generalized weak commutativity for one generating twisted field and one generator twist field. We also give a number of immediate consequences of these assumptions in this section. In Section 3, we prove that the weak commutativity and generalized weak commutativity mentioned above are equivalent to the convergence and commutativity of products of an arbitrary number of generating twisted fields, one twist generator field and an arbitrary number of generating fields for $V$. In Section 4, we define a twisted vertex operator map $Y_{W}^{g}$ for $W$ using convergence and commutativity above and prove our construction theorem that $W$ equipped with $Y_{W}^{g}$ is a lower-bounded generalized $g$-twisted $V$-module. In Section 5 , starting from a vector space $M$ graded by weights, $\mathbb{Z}_{2}$-fermion numbers and $g$-weights (eigenvalues of $g$ ) and a real number $B$ less than the real parts of all weights of homogeneous elements of $M$, we construct a lower-bounded generalized $g$-twisted $V$-module $\widehat{M}_{B}^{[g]}$ and prove that it satisfies a universal property. We also state in this section the consequence that every lower-bounded generalized $g$-twisted $V$-module is a quotient of a universal lowerbounded generalized $g$-twisted $V$-module.

Acknowledgments The author is grateful to Jason Saied for questions on the construction of modules for grading-restricted vertex algebras using the approach in [H2].

\section{Generating twisted fields and generator twist fields}

In this section, we introduce the basic assumptions needed in our construction theorem and state some immediate consequences.

In the present paper, we fix a grading-restricted vertex superalgebra $V$ and an automorphism $g$ of $V$. Then $V=\coprod_{\alpha \in P_{V}} V^{[\alpha]}$, where $V^{[\alpha]}$ is the generalized eigenspace for $g$ with eigenvalue $e^{2 \pi i \alpha}$ and $P_{V}$ is the subset of $\left\{\alpha \in \mathbb{C} \mid \Re(\alpha) \in[0,1), e^{2 \pi i \alpha}\right.$ is an eigenvalue of $\left.g\right\}$. By Lemma 2.5 in [H6], there exists an operator $\mathcal{L}_{g}$ with the semisimple and nilpotent parts $\mathcal{S}_{g}$ and $\mathcal{N}_{g}$, respectively, on $V$ such that $g=e^{2 \pi i \mathcal{L}_{g}}=e^{2 \pi i\left(\mathcal{S}_{g}+\mathcal{N}_{g}\right)}$ and by Proposition 2.6 in [H6], both $e^{2 \pi i \mathcal{S}_{g}}$ and $e^{2 \pi i \mathcal{N}_{g}}$ are also automorphisms of $V$. Generalized eigenvectors for $g$ are eigenvectors for $e^{2 \pi i \mathcal{S}_{g}}$ with the same eigenvalues and $\mathcal{N}_{g}$ is a derivation of $V$ (Proposition 2.6 in [H6]).

We first state our assumptions on $V$. 
Assumption 2.1 We assume that $V$ is generated by $\phi^{i}(x)=Y_{V}\left(\phi_{-1}^{i} \mathbf{1}, x\right)$ for $i \in I$, where $\phi^{i}(x)$ or $\phi_{-1}^{i} \mathbf{1}$ for $i \in I$ are homogeneous with respect to weights and $\mathbb{Z}_{2}$-fermion numbers and where $\phi_{-1}^{i}$ is the constant term of $\phi^{i}(x)$ and $\phi_{-1}^{i} \mathbf{1}=\lim _{x \rightarrow 0} \phi^{i}(x) \mathbf{1}$ (see [H2] for more details.) For $i \in I, \phi_{-1}^{i} \mathbf{1}$ is a generalized eigenvector of $g$ with eigenvalue $e^{2 \pi i \alpha_{i}}$. We also assume that for $i \in I$, either $\mathcal{N}_{g} \phi_{-1}^{i} \mathbf{1}=0$ or there exists $\mathcal{N}_{g}(i) \in I$ such that $\mathcal{N}_{g} \phi_{-1}^{i} \mathbf{1}=\phi_{-1}^{\mathcal{N}_{g}(i)} \mathbf{1}$.

We denote the weights and the $\mathbb{Z}_{2}$-fermion numbers of $\phi^{i}(x)$ or $\phi_{-1}^{i} \mathbf{1}$ for $i \in I$ by wt $\phi^{i}$ and $\left|\phi^{i}\right|$, respectively.

Since $\phi^{i}(x)=Y_{V}\left(\phi_{-1}^{i} \mathbf{1}, x\right)$, we have

$$
e^{2 \pi i \mathcal{S}_{g}} \phi^{i}(x) e^{-2 \pi i \mathcal{S}_{g}}=e^{2 \pi i \alpha_{i}} \phi^{i}(x)
$$

and

$$
\begin{aligned}
{\left[\mathcal{N}_{g}, \phi^{i}(x)\right] } & =\left[\mathcal{N}_{g}, Y_{V}\left(\phi_{-1}^{i} \mathbf{1}, x\right)\right] \\
& =Y_{V}\left(\mathcal{N}_{g} \phi_{-1}^{i} \mathbf{1}, x\right) .
\end{aligned}
$$

For convenience, we shall use $\phi^{0}(x)$ to denote the 0 vertex operator $Y_{V}(0, x)=0$, add 0 to the index set $I$ and denote the index set with 0 added still by $I$. Then for $i \in I$, there exists $\mathcal{N}_{g}(i) \in I$ such that $\mathcal{N}_{g} \phi_{-1}^{i} \mathbf{1}=\phi_{-1}^{\mathcal{N}_{g}(i)} \mathbf{1}$, or equivalently, $\left[\mathcal{N}_{g}, \phi^{i}(z)\right]=\phi^{\mathcal{N}_{g}(i)}(z)$. Since $\mathcal{N}_{g}$ is nilpotent, there exists $K$ such that $\mathcal{N}_{g}^{K}(i)=0$.

Next we give the data needed in our construction theorem (Theorem 4.3) in Section 4.

Data 2.2 (a) Let

$$
W=\coprod_{n \in \mathbb{C}, s \in \mathbb{Z}_{2},[\alpha] \in \mathbb{C} / \mathbb{Z}} W_{[n]}^{s ;[\alpha]}=\coprod_{n \in \mathbb{C}, s \in \mathbb{Z}_{2}, \alpha \in P_{W}} W_{[n]}^{s ;[\alpha]}
$$

be a $\mathbb{C} \times \mathbb{Z}_{2} \times \mathbb{C} / \mathbb{Z}$-graded vector space such that $W_{[n]}=\coprod_{s \in \mathbb{Z}_{2}, \alpha \in P_{W}} W_{[n]}^{s ;[\alpha]}=0$ when the real part of $n$ is sufficiently negative, where $P_{W}$ is the subset of the set $\{\alpha \in \mathbb{C} \mid \Re(\alpha) \in[0,1)\}$ such that $W_{[n]}^{s ;[\alpha]} \neq 0$ for $\alpha \in P_{W}$.

(b) Let

$$
\begin{aligned}
\phi_{W}^{i}: W & \rightarrow x^{-\alpha^{i}} W((x))[\log x] \\
w & \mapsto \phi_{W}^{i}(x) w=\sum_{k \in \mathbb{N}} \sum_{n \in \alpha^{i}+\mathbb{Z}}\left(\phi_{W}^{i}\right)_{n, k} w x^{-n-1}(\log x)^{k}
\end{aligned}
$$

for $i \in I$ be a set of linear maps called the generating twisted field maps. Since $\phi_{W}^{i}(x) w \in x^{-\alpha^{i}} W((x))[\log x]$, we must have $\left(\phi_{W}^{i}\right)_{n, k} w=0$ when $n-\alpha^{i}$ is sufficiently negative and $k$ is sufficiently large. These linear maps correspond to multivalued analytic maps with the preferred branch $\phi_{W}^{i ; 0}$ and labeled branches $\phi_{W}^{i ; p}$ for $p \in \mathbb{Z}$ from $\mathbb{C}^{\times}$to $\operatorname{Hom}(W, \bar{W})$. 
(c) Let

$$
\begin{aligned}
\psi_{W}^{a}: V & \rightarrow \sum_{\alpha \in P_{V}} x^{-\alpha} W((x))[\log x] \\
v & \mapsto \phi_{W}^{a}(x) v=\sum_{k \in \mathbb{N}} \sum_{\alpha \in P_{V}, n \in \alpha+\mathbb{Z}}\left(\psi_{W}^{a}\right)_{n, k} v x^{-n-1}(\log x)^{k}
\end{aligned}
$$

for $a \in A$ be a set of linear maps called the generator twist field maps such that $\phi_{W}^{a}(x) v \in x^{-\alpha} W((x))[\log x]$ for $\alpha \in P_{V}$ and $v \in V^{[\alpha]}$. Since $\phi_{W}^{a}(x) v \in x^{-\alpha} W((x))[\log x]$ for $v \in V^{[\alpha]}$, we must have $\left(\psi_{W}^{a}\right)_{n, k} v=0$ when $n-\alpha$ is sufficiently negative and $k$ is sufficiently large. These linear maps corresponds to multivalued analytic maps with preferred branch $\psi^{a ; 0}$ and labeled branches $\psi^{a ; p}$ for $p \in \mathbb{Z}$ from $\mathbb{C}^{\times}$to $\operatorname{Hom}(V, \bar{W})$.

(d) Let $L_{W}(0)$ and $L_{W}(-1)$ be operators on $W$.

(e) An action of $g$ on $W$, denoted still by $g$, and an operator, still denoted by $\mathcal{L}_{g}$ and its semisimple and nilpotent parts, still denoted by $\mathcal{S}_{g}$ and $\mathcal{N}_{g}$, respectively, on $W$ such that $g=e^{2 \pi i \mathcal{L}_{g}}=e^{2 \pi i\left(\mathcal{S}_{g}+\mathcal{N}_{g}\right)}$ on $W$.

These data are assumed to satisfy the following properties:

Assumption 2.3 The space $W$, the generating twisted field maps $\phi_{W}^{i}$ for $i \in I$, the generator twist field maps $\psi_{W}^{a}$ for $a \in A$, the operators $L_{W}(0), L_{W}(-1), g, \mathcal{L}_{g}, \mathcal{S}_{g}$ and $\mathcal{N}_{g}$ on $W$ in Data 2.2 have the following properties:

1. There exist semisimple and nilpotent operators $L_{W}(0)_{S}$ and $L_{W}(0)_{N}$ on $W$ such that $L_{W}(0)=L_{W}(0)_{S}+L_{W}(0)_{N}$. For $i \in I,\left[L_{W}(0), \phi_{W}^{i}(x)\right]=z \frac{d}{d x} \phi_{W}^{i}(x)+\left(\operatorname{wt} \phi^{i}\right) \phi_{W}^{i}(x)$. For $a \in A$, there exists $\left(\right.$ wt $\left.\psi_{W}^{a}\right) \in \mathbb{C}$ and, when $L_{W}(0)_{N} \psi_{W}^{a}(x) \neq 0$, there exists $L_{W}(0)_{N}(a) \in A$ such that $L_{W}(0) \psi_{W}^{a}(x)-\psi^{a}(x) L_{V}(0)=x \frac{d}{d x} \psi_{W}^{a}(x)+\left(\operatorname{wt} \psi_{W}^{a}\right) \psi_{W}^{a}(x)+$ $\psi_{W}^{L_{W}(0)_{N}(a)}(x)$, where $\psi_{W}^{L_{W}(0)_{N}(a)}(x)=0$ when $L_{W}(0)_{N} \psi_{W}^{a}(x)=0$.

2. For $i \in I,\left[L_{W}(-1), \phi_{W}^{i}(x)\right]=\frac{d}{d x} \phi_{W}^{i}(x)$ and for $a \in A, L_{W}(-1) \psi_{W}^{a}(x)-\psi_{W}^{a}(x) L_{V}(-1)=$ $\frac{d}{d x} \psi_{W}^{a}(x)$.

3. For $a \in A, \psi_{W}^{a}(x) \mathbf{1} \in W[[x]]$ and its constant terms $\lim _{x \rightarrow 0} \psi_{W}^{a}(x) \mathbf{1}$ is homogeneous with respect to weights, $\mathbb{Z}_{2}$-fermion number and $g$-weights.

4. The vector space $W$ is spanned by elements of the form $\left(\phi_{W}^{i_{1}}\right)_{n_{1}, l_{1}} \cdots\left(\phi_{W}^{i_{k}}\right)_{n_{k}, l_{k}}\left(\psi_{W}^{a}\right)_{n, l} v$ for $i_{1}, \ldots, i_{k} \in I, a \in \mathcal{A}$ and $n_{1} \in \alpha^{i_{1}}+\mathbb{Z}, \ldots, n_{k} \in \alpha^{i_{k}}+\mathbb{Z}, n \in \mathbb{C}, l_{1}, \ldots, l_{k}, l \in \mathbb{N}$, $v \in V$.

5. (i) For $i \in I, g \phi_{W}^{i ; p+1}(z) g^{-1}=\phi_{W}^{i ; p}(z)$. (ii) For $i \in I, \phi_{W}^{i}(x)=x^{-\mathcal{N}_{g}}\left(\phi_{W}^{i}\right)_{0}(x) x^{\mathcal{N}_{g}}$ and for $a \in A, \psi_{W}^{a}(x)=\left(\psi_{W}^{a}\right)_{0}(x) x^{-\mathcal{N}_{g}}$ where $\left(\phi_{W}^{i}\right)_{0}(x)$ and $\left(\psi_{W}^{a}\right)_{0}(x)$ are the constant terms of $\phi_{W}^{i}(x)$ and $\psi_{W}^{a}(x)$, respectively, viewed as power series of $\log x$ (with coefficients being series in powers of $x$ ). (iii) For $i \in I, e^{2 \pi i \mathcal{S}_{g}} \phi_{W}^{i}(z) e^{-2 \pi i \mathcal{S}_{g}}=e^{2 \pi \alpha^{i}} \phi_{W}^{i}(z)$ and $\left[\mathcal{N}_{g}, \phi_{W}^{i}(z)\right]=\phi_{W}^{\mathcal{N}_{g}(i)}(z)$. (iv) For $a \in A$, there exists $\alpha^{a} \in P_{W}$ such that $\left(\psi_{W}^{a}\right)_{n, 0} \mathbf{1}$ for $n \in-\mathbb{N}-1$ are generalized eigenvectors of $g$ with eigenvalue $e^{2 \pi i \alpha^{a}}$. 
6. For $i, j \in I$, there exists $M_{i j} \in \mathbb{Z}_{+}$such that

$$
\left(x_{1}-x_{2}\right)^{M_{i j}} \phi_{W}^{i}\left(x_{1}\right) \phi_{W}^{j}\left(x_{2}\right)=\left(x_{1}-x_{2}\right)^{M_{i j}}(-1)^{\left|\phi^{i}\right|\left|\phi^{j}\right|} \phi_{W}^{j}\left(x_{2}\right) \phi_{W}^{i}\left(x_{1}\right) .
$$

7. For $i \in I$ and $a \in A$, there exists $M_{i a} \in \mathbb{Z}_{+}$such that

$$
\begin{aligned}
& \left(x_{1}-x_{2}\right)^{\alpha_{i}+M_{i a}}\left(x_{1}-x_{2}\right)^{\mathcal{N}_{g}} \phi_{W}^{i}\left(x_{1}\right)\left(x_{1}-x_{2}\right)^{-\mathcal{N}_{g}} \psi_{W}^{a}\left(x_{2}\right) \\
& \quad=\left(-x_{2}+x_{1}\right)^{\alpha_{i}+M_{i a}}(-1)^{\left|\phi^{i}\right|\left|\psi^{a}\right|} \psi_{W}^{a}\left(x_{2}\right)\left(-x_{2}+x_{1}\right)^{\mathcal{N}_{g}} \phi^{i}\left(x_{1}\right)\left(-x_{2}+x_{1}\right)^{-\mathcal{N}_{g}} .
\end{aligned}
$$

For a multivalued analytic function $\phi(z)$ with a preferred branch of $z$ with domain $\mathbb{C}^{\times}$, we use $\phi^{p}\left(e^{a} z\right)$ to denote composition of the single-valued analytic function of $l_{p}(z)$ given by the branch $\phi^{p}(z)$ and the analytic map given by $l_{p}(z) \mapsto l_{p}(z)+a$.

We have some immediate consequences:

Proposition 2.4 The space $W$, the maps $\phi_{W}^{i}$ for $i \in I, \psi_{W}^{a}$ for $a \in A, L_{W}(-1)$ have the following properties:

8. For $c \in \mathbb{C}, i \in I$ and $a \in A$,

$$
e^{c L_{W}^{g}(0)} \phi_{W}^{i ; p}(z) e^{-c L_{V}(0)}=e^{c\left(\mathrm{wt} \phi^{i}\right)} \phi_{W}^{i ; p}\left(e^{c} z\right)
$$

and

$$
e^{c L_{W}^{g}(0)} \psi^{a ; p}(z) e^{-c L_{V}(0)}=e^{c\left(\operatorname{wt} \psi_{W}^{a}\right)} \psi^{a ; p}\left(e^{c} z\right)
$$

9. For $i_{1}, \ldots, i_{k} \in I, a \in A$ and $n_{1}, \ldots, n_{k} \in \mathbb{C}, l_{1}, \ldots, l_{k}, l \in \mathbb{N}, n \in \mathbb{Z}$ and $v \in V$, we have

$$
\begin{aligned}
L_{W}(0)\left(\phi_{W}^{i_{1}}\right)_{n_{1}, l_{1}} \cdots\left(\phi_{W}^{i_{k}}\right)_{n_{k}, l_{k}}\left(\psi_{W}^{a}\right)_{n, l} v \\
=\sum_{j=1}^{k}\left(\phi_{W}^{i_{1}}\right)_{n_{1}, l_{1}} \cdots\left(\phi_{W}^{i_{j-1}}\right)_{n_{j-1}, l_{j-1}} \cdot \\
\quad \cdot\left(\left(-n_{j}-1\right)\left(\phi_{W}^{i_{j}}\right)_{n_{j}, l_{j}}+\left(l_{j}+1\right)\left(\phi_{W}^{i_{j}}\right)_{n_{j}, l_{j}+1}+\left(\operatorname{wt} \phi^{i_{j}}\right)\left(\phi_{W}^{i_{j}}\right)_{n_{j}, l_{j}}\right) \cdot \\
\quad \cdot\left(\phi_{W}^{i_{j+1}}\right)_{n_{j+1}, l_{j+1}} \cdots\left(\phi_{W}^{i_{k}}\right)_{n_{k}, l_{k}}\left(\psi_{W}^{a}\right)_{n, l} v \\
+\left(\phi_{W}^{i_{1}}\right)_{n_{1}, l_{1}} \cdots\left(\phi_{W}^{i_{k}}\right)_{n_{k}, l_{k}} \cdot \\
\quad \cdot\left((-n-1)\left(\psi_{W}^{a}\right)_{n, l}+(l+1)\left(\psi_{W}^{a}\right)_{n, l+1}+\left(\operatorname{wt} \psi_{W}^{a}\right)\left(\psi_{W}^{a}\right)_{n, l}+\left(\psi_{W}^{L_{W}(0)_{N}(a)}\right)_{n, l}\right) v \\
+\left(\phi_{W}^{i_{1}}\right)_{n_{1}, l_{1}} \cdots\left(\phi_{W}^{i_{k}}\right)_{n_{k}, l_{k}}\left(\psi_{W}^{a}\right)_{n, l} L_{V}(0) v,
\end{aligned}
$$


and

$$
\begin{aligned}
& L_{W}(-1)\left(\phi_{W}^{i_{1}}\right)_{n_{1}, l_{1}} \cdots\left(\phi_{W}^{i_{k}}\right)_{n_{k}, l_{k}}\left(\psi_{W}^{a}\right)_{n, l} v \\
& =\sum_{j=1}^{k}\left(\phi_{W}^{i_{1}}\right)_{n_{1}, l_{1}} \cdots\left(\phi_{W}^{i_{j-1}}\right)_{n_{j-1}, l_{j-1}} \cdot \\
& \quad \cdot\left(-n_{j}\left(\phi_{W}^{i_{j}}\right)_{n_{j}-1, l_{j}}+\left(l_{j}+1\right)\left(\phi_{W}^{i_{j}}\right)_{n_{j}-1, l_{j}+1}\right)\left(\phi_{W}^{i_{j+1}}\right)_{n_{j+1}, l_{j+1}} \cdots\left(\phi_{W}^{i_{k}}\right)_{n_{k}, j_{k}}\left(\psi_{W}^{a}\right)_{n, l} v \\
& \quad+\left(\phi_{W}^{i_{1}}\right)_{n_{1}, l_{1}} \cdots\left(\phi_{W}^{i_{k}}\right)_{n_{k}, l_{k}}\left(-n\left(\psi_{W}^{a}\right)_{n-1, l}+(l+1)\left(\psi_{W}^{a}\right)_{n-1, l+1}\right) v \\
& \quad+\left(\phi_{W}^{i_{1}}\right)_{n_{1}, l_{1}} \cdots\left(\phi_{W}^{i_{k}}\right)_{n_{k}, l_{k}}\left(\psi_{W}^{a}\right)_{n, l} L_{V}(-1) v .
\end{aligned}
$$

10. For $c \in \mathbb{C}, z \in \mathbb{C}^{\times}$satisfying $|z|>|c|, i \in I$ and $a \in A, e^{c L_{W}(-1)} \phi_{W}^{i ; p}(z) e^{-c L_{W}(-1)}=$ $\phi_{W}^{i ; p}(z+c)$ and $e^{c L_{W}(-1)} \psi^{a ; p}(z) e^{-c L_{V}(-1)}=\psi^{a ; p}(z+c)$.

11. The operator $L_{W}(-1)$ has weight 1 and its adjoint $L_{W}(-1)^{\prime}$ as an operator on $W^{\prime}$ has weight -1 . In particular, $e^{z L_{W}(-1)^{\prime}} w^{\prime} \in W^{\prime}$ for $z \in \mathbb{C}$ and $w^{\prime} \in W^{\prime}$.

12. For $i \in I$, there exists $K, N \in \mathbb{N}$ such that $\phi_{W}^{i}(x)=\sum_{k=0}^{K} \sum_{n \in \alpha^{i}+N-\mathbb{N}}\left(\phi_{W}^{i}\right)_{n, k} x^{-n-1}(\log x)^{k}$ and for $i \in I$ and $w^{\prime} \in W^{\prime},\left\langle w^{\prime}, \phi_{W}^{i}(x) \cdot\right\rangle$ has only finitely many terms containing $x^{-\alpha^{i}+n}$ for $n \in \mathbb{Z}_{+}$. For $a \in A$ and $v_{1}, \ldots, v_{l}, v \in V, \psi_{W}^{a}(x) Y_{V}\left(v_{1}, x_{1}\right) \cdots Y_{V}\left(v_{l}, x_{l}\right) v$ is a polynomial in $\log x$ and for $a \in A, w^{\prime} \in W^{\prime}$ and $\alpha \in P_{V},\left\langle w^{\prime}, \psi_{W}^{a}(x) \cdot\right\rangle$ as a formal series in $x$ with coefficients in $\left(V^{[\alpha]}\right)^{*}[[\log x]]$ is of the form $\sum_{n \in \alpha+N-\mathbb{N}} \lambda_{n}(\log x) x^{-n-1}$ for some $N \in \mathbb{N}$ and $\lambda_{n} \in\left(V^{[\alpha]}\right)^{*}[[\log x]]$.

Proof. These properties follow immediately from Assumption 2.1, Data 2.2 and Properties 17 in Assumption 2.3.

Remark 2.5 The twist fields $\psi_{W}^{a}$ might look mysterious to the reader but are in fact crucial in the present paper. The reason why they are important for the construction of twisted modules (and in particular, modules) is explained in the introduction in [H6]. We repeat the explanation here. If we start with only generating twisted fields satisfying the weak commutativity or commutativity, we can still define a twisted vertex operator map but can only prove the commutativity and weak commutativity. For twisted modules (or even modules), the associativity is the main property to be verified and is not a consequence of the weak commutativity or commutativity. If we already have a twisted module, then the twist vertex operator map studied in [H6] changes the associativity of the twisted vertex operators to the commutativity involving twisted vertex operators, twist vertex operators and vertex operators for the algebra. Thus in our construction, by introducing generator twist fields $\psi_{W}^{a}$ and assuming that the commutativity holds for the generating twisted fields, generator twist fields and generating fields for the algebra $V$, we are able to prove the associativity and construct twisted modules using the same method as in the first construction of gradingrestricted vertex algebras in [H2]. 


\section{Convergence and commutativity}

The construction theorem in the next section uses the approach developed in [H2]. In this approach, the twisted vertex operators shall be defined and proved to be well defined using the correlation functions obtained from the product of the generating twisted fields $\phi_{W}^{i}(x)$, the generator twist fields $\psi_{W}^{a}(x)$ and the vertex operators for $V$. We also need the commutativity of these correlation functions. Therefore to use this approach, we first have to prove the convergence and commutativity of these products. In this section, we prove these properties.

We first give the convergence and commutativity for $\phi_{W}^{i}$. In fact, we show that the convergence and commutativity for $\phi_{W}^{i}$ are equivalent to Property 6 in Assumption 2.3 .

Theorem 3.1 Let $W=\coprod_{n \in \mathbb{C}, s \in \mathbb{Z}_{2}, \alpha \in \mathbb{C} / \mathbb{Z}} W_{[n]}^{s ;[\alpha]}$ be a $\mathbb{C} \times \mathbb{Z}_{2} \times \mathbb{C} / \mathbb{Z}$-graded vector space as in Data 2.2 and let

$$
\begin{aligned}
\phi_{W}^{i}: W & \rightarrow x^{-\alpha^{i}} W((x))[\log x] \\
w & \mapsto \phi_{W}^{i}(x) w
\end{aligned}
$$

for $i \in I$ be a set of linear maps as in Data 2.2. Assume that they satisfy the parts for $\phi_{W}^{i}$ for $i \in I$ in Properties 1, 2, (5) in Assumption [2.3. Then Property 6 in Assumption [2.3 is equivalent to the following properties:

13. For $w^{\prime} \in W^{\prime}, w \in W$ and $i_{1}, \ldots, i_{k} \in I$, the series

$$
\left\langle w^{\prime}, \phi_{W}^{i_{1} ; p}\left(z_{1}\right) \cdots \phi_{W}^{i_{k} ; p}\left(z_{k}\right) w\right\rangle
$$

is absolutely convergent in the region $\left|z_{1}\right|>\cdots>\left|z_{k}\right|>0$. Moreover, there exists a multivalued analytic function of the form

$$
\sum_{n_{1}, \ldots, n_{k}=0}^{N} f_{n_{1} \cdots n_{k+l}}\left(z_{1}, \ldots, z_{k}\right) z_{1}^{-\alpha_{i_{1}}} \cdots z_{k}^{-\alpha_{i_{k}}}\left(\log z_{1}\right)^{n_{1}} \cdots\left(\log z_{k}\right)^{n_{k}}
$$

denoted by

$$
F\left(\left\langle w^{\prime}, \phi_{W}^{i_{1}}\left(z_{1}\right) \cdots \phi_{W}^{i_{k}}\left(z_{k}\right) w\right\rangle\right),
$$

where $N \in \mathbb{N}$ and $f_{n_{1} \cdots n_{k}}\left(z_{1}, \ldots, z_{k}\right)$ for $n_{1}, \cdots, n_{k}=0, \ldots, N$ are rational functions of $z_{1}, \ldots, z_{k}$ with the only possible poles $z_{i}=0$ for $i=1, \ldots, k, z_{i}-z_{j}=0$ for $i, j=1, \ldots, k, i \neq j$, such that its sum is equal to the branch

$$
\begin{aligned}
& F^{p}\left(\left\langle w^{\prime}, \phi_{W}^{i_{1}}\left(z_{1}\right) \cdots \phi_{W}^{i_{k}}\left(z_{k}\right) w\right\rangle\right) \\
& \quad=\sum_{n_{1}, \ldots, n_{k}=0}^{N} f_{n_{1} \cdots n_{k}}\left(z_{1}, \ldots, z_{k}\right) e^{-\alpha_{i_{1}} l_{p}\left(z_{1}\right)} \cdots e^{-\alpha_{i_{k}} l_{p}\left(z_{k}\right)}\left(l_{p}\left(z_{1}\right)\right)^{n_{1}} \cdots\left(l_{p}\left(z_{k}\right)\right)^{n_{k}},
\end{aligned}
$$

of $F\left(\left\langle w^{\prime}, \phi_{W}^{i_{1}}\left(z_{1}\right) \cdots \phi_{W}^{i_{k}}\left(z_{k}\right) w\right\rangle\right)$ in the region given by $\left|z_{1}\right|>\cdots>\left|z_{k}\right|>0$. In addition, the orders of the pole $z_{i}=0$ of the rational functions $f_{n_{1} \cdots n_{k}}\left(z_{1}, \ldots, z_{k}\right)$ have a lower 
bound independent of $\phi^{i_{l}}$ for $i \neq i$ and $w^{\prime}$; the orders of the pole $z_{i}=z_{j}$ of the rational functions $f_{n_{1} \cdots n_{k}}\left(z_{1}, \ldots, z_{k}\right)$ have a lower bound independent of $\phi^{i_{l}}$ for $l \neq i, j, w$ and $w^{\prime}$.

14. For $w \in W, w^{\prime} \in W^{\prime}, i_{1}, i_{2}, i \in I$,

$$
F^{p}\left(\left\langle w^{\prime}, \phi_{W}^{i_{1}}\left(z_{1}\right) \phi_{W}^{i_{2}}\left(z_{2}\right) w\right\rangle\right)=(-1)^{\left|\phi^{i_{1}}\right|\left|\phi^{i_{2}}\right|} F^{p}\left(\left\langle v^{\prime}, \phi_{W}^{i_{2}}\left(z_{2}\right) \phi_{W}^{i_{1}}\left(z_{1}\right) w\right\rangle\right) .
$$

Proof. The proof that Properties 13 and 14 implies Property $[6$ in Assumption 2.3 is completely the same as the proof of Proposition 3.7 in [H6]. The proof that Property [6 in Assumption 2.3 implies Property 13 is completely the same as the proof of Theorem 3.10 in [H6]. We refer the reader to those proofs in [H6].

Propertiy 14 follows immediately from Property 13 in the case $k=2$ and (2.1). Thus Property 6 in Assumption 2.3 also implies Property 14.

The result below is the most general convergence result and the commutativity involving generator twist fields. We in fact prove that these convergence and commutativity properties are equivalent to Property 7 in Assumption 2.3 and thus by Theorem 3.1, together with the convergence and commutativity properties in Theorem 3.1, are equivalent to Properties 6 and 7 in Assumption 2.3 .

Theorem 3.2 Let $W=\coprod_{n \in \mathbb{C}, s \in \mathbb{Z}_{2}, \alpha \in \mathbb{C} / \mathbb{Z}} W_{[n]}^{s ;[\alpha]}$ be a $\mathbb{C} \times \mathbb{Z}_{2} \times \mathbb{C} / \mathbb{Z}$-graded vector space as in Data 2.2 and let

$$
\begin{aligned}
\phi_{W}^{i}: W & \rightarrow x^{-\alpha^{i}} W((x))[\log x] \\
w & \mapsto \phi_{W}^{i}(x) w
\end{aligned}
$$

for $i \in I$ and

$$
\begin{aligned}
\psi_{W}^{a}: V & \rightarrow \sum_{\alpha \in P_{V}} x^{-\alpha} W((x))[\log x] \\
v & \mapsto \phi_{W}^{a}(x) v
\end{aligned}
$$

for $a \in A$ be linear maps as in Data [2.2. Assume that they satisfy Properties 11 6 in Assumption 2.3. Then Property 1 is equivalent to the following properties:

15. For $w^{\prime} \in W^{\prime}, v \in V^{[\alpha]}$ and $i_{1}, \ldots, i_{k+l} \in I, a \in A$, the series

$$
\left\langle w^{\prime}, \phi_{W}^{i_{1} ; p}\left(z_{1}\right) \cdots \phi_{W}^{i_{k} ; p}\left(z_{k}\right) \psi^{a ; p}(z) \phi^{i_{k+1}}\left(z_{k+1}\right) \cdots \phi^{i_{k+l}}\left(z_{k+l}\right) v\right\rangle
$$

is absolutely convergent in the region $\left|z_{1}\right|>\cdots>\left|z_{k}\right|>|z|>\left|z_{k+1}\right|>\cdots>\left|z_{k+l}\right|>0$. Moreover, there exists a multivalued analytic function of the form

$$
\begin{aligned}
& \sum_{n_{1}, \ldots, n_{k+l}, n=0}^{N} f_{n_{1} \cdots n_{k+l} n}\left(z_{1}, \ldots, z_{k+l}, z\right) . \\
& \quad \cdot\left(z_{1}-z\right)^{-\alpha_{i_{1}}} \cdots\left(z_{k+l}-z\right)^{-\alpha_{i_{k+l}}} z^{-\alpha}\left(\log \left(z_{1}-z\right)\right)^{n_{1}} \cdots\left(\log \left(z_{k+l}-z\right)\right)^{n_{k+l}}(\log z)^{n},
\end{aligned}
$$


denoted by

$$
F\left(\left\langle w^{\prime}, \phi_{W}^{i_{1}}\left(z_{1}\right) \cdots \phi_{W}^{i_{k}}\left(z_{k}\right) \psi_{W}^{a}(z) \phi^{i_{k+1}}\left(z_{k+1}\right) \cdots \phi^{i_{k+l}}\left(z_{k+l}\right) v\right\rangle\right)
$$

where $N \in \mathbb{N}$ and $f_{n_{1} \cdots n_{k+l} n}\left(z_{1}, \ldots, z_{k+l}, z\right)$ for $n_{1}, \cdots, n_{k+l}, n=0, \ldots, N$ are rational functions of $z_{1}, \ldots, z_{k+l}, z$ with the only possible poles $z_{i}=0$ for $i=1, \ldots, k+l, z=0$, $z_{i}-z_{j}=0$ for $i, j=1, \ldots, k+l, i \neq j, z_{i}-z=0$ for $i=1, \ldots, k+l$, such that its sum is equal to the branch

$$
\begin{aligned}
& F^{p}\left(\left\langle w^{\prime}, \phi_{W}^{i_{1}}\left(z_{1}\right) \cdots \phi_{W}^{i_{k}}\left(z_{k}\right) \psi_{W}^{a}(z) \phi^{i_{k+1}}\left(z_{k+1}\right) \cdots \phi^{i_{k+l}}\left(z_{k+l}\right) v\right\rangle\right) \\
& =\sum_{n_{1}, \ldots, n_{k+l}, n=0}^{N} f_{n_{1} \cdots n_{k+l} n}\left(z_{1}, \ldots, z_{k+l}\right) \cdot \\
& \quad \cdot e^{-\alpha_{i_{1}} l_{p}\left(z_{1}-z\right)} \cdots e^{-\alpha_{i_{k+l}} l_{p}\left(z_{k+l}-z\right)} e^{-\alpha l_{p}(z)}\left(l_{p}\left(z_{1}-z\right)\right)^{n_{1}} \cdots\left(l_{p}\left(z_{k+l}-z\right)\right)^{n_{k+l}}\left(l_{p}(z)\right)^{n}
\end{aligned}
$$

of (3.2) in the region given by $\left|z_{1}\right|>\cdots>\left|z_{k}\right|>|z|>\left|z_{k+1}\right|>\cdots>\left|z_{k+l}\right|>0$, $\left|\arg \left(z_{i}-z\right)-\arg z_{i}\right|<\frac{\pi}{2}$ for $i=1, \ldots, k$ and $\left|\arg \left(z_{i}-z\right)-\arg z\right|<\frac{\pi}{2}$ for $i=$ $k+1, \ldots, k+l$. In addition, the orders of the pole $z_{j}=0$ of the rational functions $f_{n_{1} \cdots n_{k+l} n}\left(z_{1}, \ldots, z_{k+l}, z\right)$ have a lower bound independent of $v_{q}$ for $q \neq j, w$ and $w^{\prime}$; the orders of the pole $z=0$ of the rational functions $f_{n_{1} \cdots n_{k+l} n}\left(z_{1}, \ldots, z_{k+l}, z\right)$ have a lower bound independent of $v_{1}, \ldots, v_{k+l}$ and $w^{\prime}$; the orders of the pole $z_{j}=z_{m}$ of the rational functions $f_{n_{1} \cdots n_{k+l} n}\left(z_{1}, \ldots, z_{k+l}, z\right)$ have a lower bound independent of $v_{q}$ for $q \neq j, m, v, w$ and $w^{\prime}$; the orders of the pole $z_{j}=z$ of the rational functions $f_{n_{1} \cdots n_{k+l} n}\left(z_{1}, \ldots, z_{k+l}, z\right)$ have a lower bound independent of $v_{q}$ for $q \neq j$, $v$ and $w^{\prime}$.

16. For $v \in V, w^{\prime} \in W^{\prime}, i \in I, a \in A$,

$$
F^{p}\left(\left\langle w^{\prime}, \phi_{W}^{i}\left(z_{1}\right) \psi_{W}^{a}\left(z_{2}\right) v\right\rangle\right)=(-1)^{\left|\phi^{i}\right|\left|\psi_{W}^{a}\right|} F^{p}\left(\left\langle w^{\prime}, \psi_{W}^{a}\left(z_{2}\right) \phi^{i}\left(z_{1}\right) v\right\rangle\right) .
$$

In particular, when Properties 15 in Assumption 2.3 hold, Properties 6 and 7 in Assumption 2.3 are equivalent to Properties 13 and 14 in Theorem 3.1 and Properties 15 and 16.

Proof. Assume that Properties 15 and 16 hold. For $w^{\prime} \in W^{\prime}$ and $v \in V$, by Property 5 in Assumption 2.3,

$$
\begin{aligned}
\left\langle w^{\prime}, \phi_{W}^{i ; p}\left(z_{1}\right) \psi_{W}^{a ; p}\left(z_{2}\right) v\right\rangle & =\left\langle w^{\prime}, e^{-l_{p}\left(z_{1}\right) \mathcal{N}_{g}}\left(\phi_{W}^{i}\right)_{0}^{p}\left(z_{1}\right) e^{l_{p}\left(z_{1}\right) \mathcal{N}_{g}} \psi_{W}^{a ; p}\left(z_{2}\right) v\right\rangle \\
& \left.=\sum_{r \in \mathbb{N}} \frac{(-1)^{r}}{r !}\left(l_{p}\left(z_{1}\right)\right)^{r}\left\langle w^{\prime},[\overbrace{\mathcal{N}_{g}, \cdots,\left[\mathcal{N}_{g}\right.}^{r},\left(\phi_{W}^{i}\right)_{0}^{p}\left(z_{1}\right)] \cdots\right] \psi_{W}^{a ; p}\left(z_{2}\right) v\right\rangle \\
& =\sum_{r \in \mathbb{N}} \frac{(-1)^{r}}{r !}\left\langle w^{\prime},\left(\phi_{W}^{\mathcal{N}_{g}^{r}(i)}\right)_{0}^{p}\left(z_{1}\right) \psi_{W}^{a ; p}\left(z_{2}\right) v\right\rangle\left(l_{p}\left(z_{1}\right)\right)^{r},
\end{aligned}
$$


where, in our notation, for $j \in I,\left(\phi_{W}^{j}\right)_{0}^{p}\left(z_{1}\right)$ denotes the $p$-th branch of $\left(\phi_{W}^{j}\right)_{0}\left(z_{1}\right)$. By Property 15, the left-hand side of (3.5) is absolutely convergent in the region given by $\left|z_{1}\right|>\left|z_{2}\right|>0$ and $\left|\arg \left(z_{1}-z_{2}\right)-\arg z_{1}\right|<\frac{\pi}{2}$ to

$$
\sum_{j, k, l, q=0}^{N} a_{q j k l} z_{1}^{-t} e^{-\left(\alpha_{i}+m_{q}\right) l_{p}\left(z_{1}-z_{2}\right)} e^{n_{j} l_{p}\left(z_{2}\right)}\left(l_{p}\left(z_{1}-z_{2}\right)\right)^{k}\left(l_{p}\left(z_{2}\right)\right)^{l}
$$

where $t, m_{q} \in \mathbb{Z}$ for $q=0, \ldots, N$ and $n_{j} \in \mathbb{C}$ for $j=0, \ldots, N$. But the expansion of (3.6) in the region given by $\left|z_{1}\right|>\left|z_{2}\right|>0$ and $\left|\arg \left(z_{1}-z_{2}\right)-\arg z_{1}\right|<\frac{\pi}{2}$ as a power series in $l_{p}\left(z_{1}\right)$ is

$$
\begin{aligned}
& \sum_{k, j, l, q=0}^{N} a_{q j k l} z_{1}^{-t} e^{-\left(\alpha_{i}+m_{q}\right) l_{p}\left(z_{1}-z_{2}\right)} e^{n_{j} l_{p}\left(z_{2}\right)}\left(l_{p}\left(z_{2}\right)\right)^{l} \sum_{r=0}^{k}\left(\begin{array}{l}
k \\
r
\end{array}\right)\left(\log \left(1-\frac{z_{2}}{z_{1}}\right)\right)^{k-r}\left(l_{p}\left(z_{1}\right)\right)^{r} \\
& =\sum_{r=1}^{N} \sum_{k=r}^{N} \sum_{j, l, q=0}^{N} a_{q j k l} z_{1}^{-t} e^{-\left(\alpha_{i}+m_{q}\right) l_{p}\left(z_{1}-z_{2}\right)} e^{n_{j} l_{p}\left(z_{2}\right)}\left(l_{p}\left(z_{2}\right)\right)^{l}\left(\begin{array}{l}
k \\
r
\end{array}\right)\left(\log \left(1-\frac{z_{2}}{z_{1}}\right)\right)^{k-r}\left(l_{p}\left(z_{1}\right)\right)^{r},
\end{aligned}
$$

where $\left(\log \left(1-\frac{z_{2}}{z_{1}}\right)\right)^{k-r}$ is understood as the branch obtained by using the expansion in nonnegative powers of $\frac{z_{2}}{z_{1}}$. Comparing (3.6) and (3.7) and using Proposition 2.1 in [H4], we obtain

$$
\begin{aligned}
& \frac{(-1)^{r}}{r !}\left\langle w^{\prime},\left(\phi_{W}^{\mathcal{N}_{g}^{r}(i)}\right)_{0}^{p}\left(z_{1}\right) \psi_{W}^{a ; p}\left(z_{2}\right) v\right\rangle \\
& \quad=\sum_{k=r}^{N} \sum_{j, l, q=0}^{N} a_{q j k l} z_{1}^{-t} e^{-\left(\alpha_{i}+m_{q}\right) l_{p}\left(z_{1}-z_{2}\right)} e^{n_{j} l_{p}\left(z_{2}\right)}\left(l_{p}\left(z_{2}\right)\right)^{l}\left(\begin{array}{l}
k \\
r
\end{array}\right)\left(\log \left(1-\frac{z_{2}}{z_{1}}\right)\right)^{k-r}
\end{aligned}
$$

for $r \in \mathbb{N}$ and for $r>N$, both sides of (3.8) are 0 . Then in the region given by $\left|z_{1}\right|>\left|z_{2}\right|>0$ 
and $\left|\arg \left(z_{1}-z_{2}\right)-\arg z_{1}\right|<\frac{\pi}{2}$

$$
\begin{aligned}
& \left\langle w^{\prime}, e^{l_{p}\left(z_{1}-z_{2}\right) \mathcal{N}_{g}} \phi_{W}^{i ; p}\left(z_{1}\right) e^{-l_{p}\left(z_{1}-z_{2}\right) \mathcal{N}_{g}} \psi_{W}^{a ; p}\left(z_{2}\right) v\right\rangle \\
& =\left\langle w^{\prime}, e^{l_{p}\left(z_{1}-z_{2}\right) \mathcal{N}_{g}} e^{-l_{p}\left(z_{1}\right) \mathcal{N}_{g}}\left(\phi_{W}^{i}\right)_{0}^{p}\left(z_{1}\right) e^{l_{p}\left(z_{1}\right) \mathcal{N}_{g}} e^{-l_{p}\left(z_{1}-z_{2}\right) \mathcal{N}_{g}} \psi_{W}^{a ; p}\left(z_{2}\right) v\right\rangle \\
& =\left\langle w^{\prime}, e^{\left(\log \left(1-\frac{z_{2}}{z_{1}}\right)\right) \mathcal{N}_{g}}\left(\phi_{W}^{i}\right)_{0}^{p}\left(z_{1}\right) e^{-\left(\log \left(1-\frac{z_{2}}{z_{1}}\right)\right) \mathcal{N}_{g}} \psi_{W}^{a ; p}\left(z_{2}\right) v\right\rangle \\
& \left.=\sum_{r \in \mathbb{N}} \frac{1}{r !}\left(\log \left(1-\frac{z_{2}}{z_{1}}\right)\right)^{r}\left\langle w^{\prime},[\overbrace{\mathcal{N}_{g}, \cdots,\left[\mathcal{N}_{g}\right.}^{r},\left(\phi_{W}^{i}\right)_{0}^{p}\left(z_{1}\right)] \cdots\right] \psi_{W}^{a ; p}\left(z_{2}\right) v\right\rangle \\
& =\sum_{r \in \mathbb{N}} \frac{1}{r !}\left\langle w^{\prime},\left(\phi_{W}^{\mathcal{N}_{g}^{r}(i)}\right)_{0}^{p}\left(z_{1}\right) \psi_{W}^{a ; p}\left(z_{2}\right) v\right\rangle\left(\log \left(1-\frac{z_{2}}{z_{1}}\right)\right)^{r} \\
& =\sum_{r=1}^{N} \sum_{k=r}^{N} \sum_{j, l, q=0}^{N}(-1)^{r} a_{q j k l} z_{1}^{-t} e^{-\left(\alpha_{i}+m_{q}\right) l_{p}\left(z_{1}-z_{2}\right)} e^{n_{j} l_{p}\left(z_{2}\right)}\left(l_{p}\left(z_{2}\right)\right)^{l} . \\
& \cdot\left(\begin{array}{l}
k \\
r
\end{array}\right)\left(\log \left(1-\frac{z_{2}}{z_{1}}\right)\right)^{k-r}\left(\log \left(1-\frac{z_{2}}{z_{1}}\right)\right)^{r} \\
& =\sum_{k=1}^{N} \sum_{j, l, q=0}^{N} a_{q j k l} z_{1}^{-t} e^{-\left(\alpha_{i}+m_{q}\right) l_{p}\left(z_{1}-z_{2}\right)} e^{n_{j} l_{p}\left(z_{2}\right)}\left(l_{p}\left(z_{2}\right)\right)^{l} . \\
& \cdot\left(\sum_{r=1}^{k}\left(\begin{array}{l}
k \\
r
\end{array}\right)\left(\log \left(1-\frac{z_{2}}{z_{1}}\right)\right)^{k-r}(-1)^{r}\left(\log \left(1-\frac{z_{2}}{z_{1}}\right)\right)^{r}\right) \\
& =\sum_{k=1}^{N} \sum_{j, l, q=0}^{N} a_{q j k l} z_{1}^{-t} e^{-\left(\alpha_{i}+m_{q}\right) l_{p}\left(z_{1}-z_{2}\right)} e^{n_{j} l_{p}\left(z_{2}\right)}\left(l_{p}\left(z_{2}\right)\right)^{l} . \\
& \cdot\left(\left(\log \left(1-\frac{z_{2}}{z_{1}}\right)\right)-\left(\log \left(1-\frac{z_{2}}{z_{1}}\right)\right)\right)^{k} \\
& =\sum_{j, l, q=0}^{N} a_{q j 0 l} z_{1}^{-t} e^{-\left(\alpha_{i}+m_{q}\right) l_{p}\left(z_{1}-z_{2}\right)} e^{n_{j} l_{p}\left(z_{2}\right)}\left(l_{p}\left(z_{2}\right)\right)^{l} .
\end{aligned}
$$

On the other hand, in the region given by $\left|z_{2}\right|>\left|z_{1}\right|>0$ and $\left|\arg \left(z_{1}-z_{2}\right)-\arg z_{2}\right|<\frac{\pi}{2}$,

$$
\begin{aligned}
(-1)^{\left|\phi^{i}\right|\left|\psi^{a}\right|}\left\langle w^{\prime}, \psi_{W}^{a ; p}\left(z_{2}\right) e^{l_{p}\left(z_{2}-z_{1}\right) \mathcal{N}_{g}} e^{\pi i \mathcal{N}_{g}} \phi^{i}\left(z_{1}\right) e^{-\pi i \mathcal{N}_{g}} e^{-l_{p}\left(z_{2}-z_{1}\right) \mathcal{N}_{g}} v\right\rangle \\
=(-1)^{\left|\phi^{i}\right|\left|\psi^{a}\right|}\left\langle w^{\prime}, \psi_{W}^{a ; p}\left(z_{2}\right) e^{\left(l_{p}\left(z_{2}-z_{1}\right)+\pi i\right) \mathcal{N}_{g}} \phi^{i}\left(z_{1}\right) e^{-\left(l_{p}\left(z_{2}-z_{1}\right)+\pi i\right) \mathcal{N}_{g}} v\right\rangle \\
\left.=\sum_{k \in \mathbb{N}} \frac{1}{k !}\left(l_{p}\left(z_{2}-z_{1}\right)+\pi i\right)^{k}(-1)^{\left|\phi^{i}\right|\left|\psi^{a}\right|}\left\langle w^{\prime}, \psi_{W}^{a ; p}\left(z_{2}\right)[\overbrace{\mathcal{N}_{g}, \cdots,\left[\mathcal{N}_{g}\right.}^{k}, \phi^{i ; p}\left(z_{1}\right)] \cdots\right] v\right\rangle \\
=\sum_{k \in \mathbb{N}} \frac{1}{k !}\left(l_{p}\left(z_{2}-z_{1}\right)+\pi i\right)^{k}(-1)^{\left|\phi^{i}\right|\left|\psi^{a}\right|}\left\langle w^{\prime}, \psi_{W}^{a ; p}\left(z_{2}\right) \phi^{\mathcal{N}_{g}^{k}(i) ; p}\left(z_{1}\right) v\right\rangle .
\end{aligned}
$$


By Property 5 in Assumption 2.3 and (3.8), in the region given by $\left|z_{1}\right|>\left|z_{2}\right|>0$ and $\left|\arg \left(z_{1}-z_{2}\right)-\arg z_{1}\right|<\frac{\pi}{2}$, we have

$$
\begin{aligned}
&\left\langle w^{\prime}, \phi_{W}^{\mathcal{N}_{g}^{k}(i) ; p}\left(z_{1}\right) \psi_{W}^{a ; p}\left(z_{2}\right) v\right\rangle \\
&=\left\langle w^{\prime}, e^{-l_{p}\left(z_{1}\right) \mathcal{N}_{g}}\left(\phi_{W}^{\mathcal{N}_{g}^{k}(i)}\right)_{0}^{p}\left(z_{1}\right) e^{-l_{p}\left(z_{1}\right) \mathcal{N}_{g}} \psi_{W}^{a ; p}\left(z_{2}\right) v\right\rangle \\
&=\left.\sum_{n \in \mathbb{N}} \frac{(-1)^{n}}{n !}\left(l_{p}\left(z_{1}\right)\right)^{n}\left\langle w^{\prime},[\overbrace{\mathcal{N}_{g}, \cdots\left[\mathcal{N}_{g}\right.}^{n},\left(\phi_{W}^{\mathcal{N}_{g}^{k}(i)}\right)_{0}^{p}\left(z_{1}\right)] \cdots\right] \psi_{W}^{a ; p}\left(z_{2}\right) v\right\rangle \\
&= \sum_{n \in \mathbb{N}} \frac{(-1)^{n}}{n !}\left(l_{p}\left(z_{1}\right)\right)^{n}\left\langle w^{\prime},\left(\phi_{W}^{\mathcal{N}_{g}^{k+n}(i)}\right)_{0}^{p}\left(z_{1}\right) \psi_{W}^{a ; p}\left(z_{2}\right) v\right\rangle \\
&= \sum_{n \in \mathbb{N}} \frac{(-1)^{k}(k+n) !}{n !}\left(l_{p}\left(z_{1}\right)\right)^{n} \sum_{s=k+n}^{N} \sum_{j, l, q=0}^{N} a_{q j s l} z_{1}^{-t} e^{-\left(\alpha_{i}+m_{q}\right) l_{p}\left(z_{1}-z_{2}\right)} e^{n_{j} l_{p}\left(z_{2}\right)}\left(l_{p}\left(z_{2}\right)\right)^{l} . \\
& \cdot\left(\begin{array}{c}
s \\
k+n
\end{array}\right)\left(\log \left(1-\frac{z_{2}}{z_{1}}\right)\right)^{s-(k+n)} \\
&=\sum_{n \in \mathbb{N}} \sum_{s=k+n}^{N} \sum_{j, l, q=0}^{N} \frac{(-1)^{k}(k+n) !}{n !} a_{q j s l} z_{1}^{-t} e^{-\left(\alpha_{i}+m_{q}\right) l_{p}\left(z_{1}-z_{2}\right)} e^{n_{j} l_{p}\left(z_{2}\right)}\left(l_{p}\left(z_{2}\right)\right)^{l} . \\
& \cdot\left(\begin{array}{c}
s \\
k+n
\end{array}\right)\left(l_{p}\left(z_{1}\right)\right)^{n}\left(\log \left(1-\frac{z_{2}}{z_{1}}\right)^{s-(k+n)}\right.
\end{aligned}
$$

By Property 16,

$$
(-1)^{\left|\phi^{i}\right|\left|\psi^{a}\right|}\left\langle w^{\prime}, \psi_{W}^{a ; p}\left(z_{2}\right) \phi_{W}^{\mathcal{N}_{g}^{k}(i) ; p}\left(z_{1}\right) v\right\rangle
$$

and the left-hand side of (3.11) converges absolutely to the same branch of a multivalued analytic function in different regions. Then we see from (3.11) that in the region given by $\left|z_{2}\right|>\left|z_{1}\right|>0$ and $\left|\arg \left(z_{1}-z_{2}\right)-\arg z_{2}\right|<\frac{\pi}{2}$, (3.12) must converge absolutely to

$$
\begin{gathered}
\sum_{n \in \mathbb{N}} \sum_{s=k+n}^{N} \sum_{j, l, q=0}^{N} \frac{(-1)^{k}(k+n) !}{n !} a_{q j s l} z_{1}^{-t} e^{-\left(\alpha_{i}+m_{q}\right) l_{p}\left(z_{1}-z_{2}\right)} e^{n_{j} l_{p}\left(z_{2}\right)}\left(l_{p}\left(z_{2}\right)\right)^{l} . \\
\cdot\left(\begin{array}{c}
s \\
k+n
\end{array}\right)\left(l_{p}\left(z_{1}\right)\right)^{n}\left(\log \left(1-\frac{z_{2}}{z_{1}}\right)\right)^{s-(k+n)} .
\end{gathered}
$$


Thus the right-hand side of (3.10) is equal to

$$
\begin{aligned}
& \sum_{k \in \mathbb{N}} \sum_{n \in \mathbb{N}} \sum_{s=k+n}^{N} \sum_{j, l, q=0}^{N} \frac{(-1)^{k}(k+n) !}{k ! n !} a_{q j s l} z_{1}^{-t} e^{-\left(\alpha_{i}+m_{q}\right) l_{p}\left(z_{1}-z_{2}\right)} e^{n_{j} l_{p}\left(z_{2}\right)}\left(l_{p}\left(z_{2}\right)\right)^{l} . \\
& \cdot\left(\begin{array}{c}
s \\
k+n
\end{array}\right)\left(l_{p}\left(z_{2}-z_{1}\right)+\pi i\right)^{k}\left(l_{p}\left(z_{1}\right)\right)^{n}\left(\log \left(1-\frac{z_{2}}{z_{1}}\right)\right)^{s-(k+n)} \\
& =\sum_{j, s, l, q=0}^{N} \sum_{r=0}^{s} a_{q j s l} z_{1}^{-t} e^{-\left(\alpha_{i}+m_{q}\right) l_{p}\left(z_{1}-z_{2}\right)} e^{n_{j} l_{p}\left(z_{2}\right)}\left(l_{p}\left(z_{2}\right)\right)^{l} . \\
& \cdot\left(\begin{array}{l}
s \\
r
\end{array}\right)\left(\sum_{k \in \mathbb{N}}\left(\begin{array}{l}
r \\
k
\end{array}\right)(-1)^{k}\left(l_{p}\left(z_{2}-z_{1}\right)+\pi i\right)^{k}\left(l_{p}\left(z_{1}\right)\right)^{s-k}\right)\left(\log \left(1-\frac{z_{2}}{z_{1}}\right)\right)^{s-r} \\
& =\sum_{j, s, l, q=0}^{N} a_{q j s l} z_{1}^{-t} e^{-\left(\alpha_{i}+m_{q}\right) l_{p}\left(z_{1}-z_{2}\right)} e^{n_{j} l_{p}\left(z_{2}\right)}\left(l_{p}\left(z_{2}\right)\right)^{l} . \\
& \cdot \sum_{r=0}^{s}\left(\begin{array}{l}
s \\
r
\end{array}\right)\left(l_{p}\left(z_{1}\right)-\left(l_{p}\left(z_{2}-z_{1}\right)+\pi i\right)\right)^{s}\left(\log \left(1-\frac{z_{2}}{z_{1}}\right)\right)^{s-r} \\
& =\sum_{j, s, l, q=0}^{N} a_{q j s l} z_{1}^{-t} e^{-\left(\alpha_{i}+m_{q}\right) l_{p}\left(z_{1}-z_{2}\right)} e^{n_{j} l_{p}\left(z_{2}\right)}\left(l_{p}\left(z_{2}\right)\right)^{l} . \\
& \cdot\left(l_{p}\left(z_{1}\right)-\left(l_{p}\left(z_{2}-z_{1}\right)+\pi i\right)+\log \left(1-\frac{z_{2}}{z_{1}}\right)\right)^{s} \\
& =\sum_{j, l, q=0}^{N} a_{q j 0 l} z_{1}^{-t} e^{-\left(\alpha_{i}+m_{q}\right) l_{p}\left(z_{1}-z_{2}\right)} e^{n_{j} l_{p}\left(z_{2}\right)}\left(l_{p}\left(z_{2}\right)\right)^{l} .
\end{aligned}
$$

From (3.9) and the calculations from $(3.10)$ to (3.14), we see that if we choose $M_{i a} \in \mathbb{Z}_{+}$ to be larger than $m_{q}$ for $q=0, \ldots, N$, we have

$$
\begin{aligned}
& e^{\left(\alpha_{i}+M_{i a}\right) l_{p}\left(z_{1}-z_{2}\right)}\left\langle w^{\prime}, e^{l_{p}\left(z_{1}-z_{2}\right) \mathcal{N}_{g}} \phi_{W}^{i ; p}\left(z_{1}\right) e^{-l_{p}\left(z_{1}-z_{2}\right) \mathcal{N}_{g}} \psi_{W}^{a ; p}\left(z_{2}\right) v\right\rangle \\
& =\sum_{j, l, q=0}^{N} a_{q j 0 l} z_{1}^{-t}\left(z_{1}-z_{2}\right)^{M_{i a}-m_{q}} e^{n_{j} l_{p}\left(z_{2}\right)}\left(l_{p}\left(z_{2}\right)\right)^{l} \\
& =e^{\left(\alpha_{i}+M_{i a}\right) l_{p}\left(z_{2}-z_{1}\right)} e^{\pi i\left(\alpha_{i}+M_{i a}\right)}(-1)^{\left|\phi^{i}\right|\left|\psi^{a}\right|} . \\
& \quad \cdot\left\langle w^{\prime}, \psi_{W}^{a ; p}\left(z_{2}\right) e^{l_{p}\left(z_{2}-z_{1}\right) \mathcal{N}_{g}} e^{\pi i \mathcal{N}_{g}} \phi^{i}\left(z_{1}\right) e^{-\pi i \mathcal{N}_{g}} e^{-l_{p}\left(z_{2}-z_{1}\right) \mathcal{N}_{g}} v\right\rangle
\end{aligned}
$$

The formula (3.15) for all $w^{\prime} \in W^{\prime}$ and $v \in V$ is equivalent to the formal identity (2.2).

Next we prove that Property 7 in Assumption 2.3 implies Properties [15] and 16. By (b) in Data 2.2 and Property 12 in Proposition 2.4, there exist $N_{1}, N_{2} \in \mathbb{Z}$ and $K \in \mathbb{N}$ such that

$$
\left\langle w^{\prime}, \phi_{W}^{i}(x) w\right\rangle=\sum_{n=N_{1}}^{N_{2}} \sum_{k=1}^{K}\left\langle w^{\prime},\left(\phi_{W}^{i}\right)_{\alpha^{i}+n, k} w\right\rangle x^{-\left(\alpha^{i}+n\right)-1}(\log x)^{k}
$$


for $w \in W$ and $w^{\prime} \in W^{\prime}$. Then for $w^{\prime} \in W^{\prime}$ and $v \in V$,

$$
\begin{gathered}
\left(x_{1}-x_{2}\right)^{\alpha_{i}+M_{i a}}\left\langle w^{\prime},\left(x_{1}-x_{2}\right)^{\mathcal{N}_{g}} \phi_{W}^{i}\left(x_{1}\right)\left(x_{1}-x_{2}\right)^{-\mathcal{N}_{g}} \psi_{W}^{a}\left(x_{2}\right) v\right\rangle \\
\in \mathbb{C}\left(\left(x_{1}^{-1}, x_{2}\right)\right)\left[\log x_{2}\right], \\
\left(x_{2}-x_{1}\right)^{\alpha_{i}+M_{i a}} e^{\pi i\left(\alpha_{i}+M_{i a}\right)}(-1)^{\left|\phi^{i} \| \psi^{a}\right|}\left\langle w^{\prime}, \psi_{W}^{a}\left(x_{2}\right)\left(x_{2}-x_{1}\right)^{\mathcal{N}_{g}} e^{\pi i \mathcal{N}_{g}} \phi^{i}\left(x_{1}\right) e^{-\pi i \mathcal{N}_{g}}\left(x_{2}-x_{1}\right)^{-\mathcal{N}_{g}} v\right\rangle \\
\in \mathbb{C}\left(\left(x_{1}, x_{2}^{-1}\right)\right)\left[\log x_{2}\right],
\end{gathered}
$$

where we use $\mathbb{C}\left(\left(x_{1}^{-1}, x_{2}\right)\right)\left(\mathbb{C}\left(\left(x_{1}, x_{2}^{-1}\right)\right)\right)$ to denote the ring of Laurent series in $x_{1}$ and $x_{2}$ having only finitely many terms in positive powers of $x_{1}$ and negative powers of $x_{2}$ (finitely many terms in negative powers of $x_{1}$ and positive powers of $x_{2}$ ). But by (2.2) these two formal series are equal. So they must belong to

$$
\mathbb{C}\left[x_{1}, x_{1}^{-1}, x_{2}, x_{2}^{-1}\right]\left[\log x_{1}, \log x_{2}\right] .
$$

This formal series can be written as

$$
\sum_{j, l, q=0}^{N} b_{q j l} x_{1}^{-t}\left(x_{1}-x_{2}\right)^{M_{i a}-m_{q}} x_{2}^{n_{j}}\left(\log \left(x_{2}\right)\right)^{l}
$$

for $b_{q j l} \in \mathbb{C}, t \in \mathbb{N}, m_{q} \in \mathbb{Z}$ such that $m_{q} \leq M_{i a}, n_{j} \in \mathbb{C}$ so that

$$
\begin{aligned}
\left(x_{1}-x_{2}\right)^{\alpha_{i}+M_{i a}}\left\langle w^{\prime},\left(x_{1}-x_{2}\right)^{\mathcal{N}_{g}} \phi_{W}^{i}\left(x_{1}\right)\left(x_{1}-x_{2}\right)^{-\mathcal{N}_{g}} \psi_{W}^{a}\left(x_{2}\right) v\right\rangle & \\
= & \sum_{j, l, q=0}^{N} b_{q j l} x_{1}^{-t}\left(x_{1}-x_{2}\right)^{M_{i a}-m_{q}} x_{2}^{n_{j}}\left(\log \left(x_{2}\right)\right)^{l} \\
= & \left(x_{2}-x_{1}\right)^{\alpha_{i}+M_{i a}} e^{\pi i\left(\alpha_{i}+M_{i a}\right)}(-1)^{\left|\phi^{i}\right|\left|\psi^{a}\right|} . \\
& \cdot\left\langle w^{\prime}, \psi_{W}^{a}\left(x_{2}\right)\left(x_{2}-x_{1}\right)^{\mathcal{N}_{g}} e^{\pi i \mathcal{N}_{g}} \phi^{i}\left(x_{1}\right) e^{-\pi i \mathcal{N}_{g}}\left(x_{2}-x_{1}\right)^{-\mathcal{N}_{g}} v\right\rangle .
\end{aligned}
$$

Thus

$$
\begin{aligned}
F^{p} & \left(\left\langle w^{\prime},\left(z_{1}-z_{2}\right)^{\mathcal{N}_{g}} \phi_{W}^{i}\left(z_{1}\right)\left(z_{1}-z_{2}\right)^{-\mathcal{N}_{g}} \psi_{W}^{a}\left(z_{2}\right) v\right\rangle\right) \\
& =\sum_{j, l, q=0}^{N} b_{q j l} z_{1}^{-t} e^{-\left(\alpha_{i}+m_{q}\right) l_{p}\left(z_{1}-z_{2}\right)} e^{n_{j} l_{p}\left(z_{2}\right)}\left(l_{p}\left(z_{2}\right)\right)^{l} \\
& =(-1)^{\left|\phi^{i}\right|\left|\psi^{a}\right|} F^{p}\left(\left\langle w^{\prime}, \psi_{W}^{a}\left(z_{2}\right)\left(z_{2}-z_{1}\right)^{\mathcal{N}_{g}} e^{\pi i \mathcal{N}_{g}} \phi^{i}\left(z_{1}\right) e^{-\pi i \mathcal{N}_{g}}\left(z_{2}-z_{1}\right)^{-\mathcal{N}_{g}} v\right\rangle\right) .
\end{aligned}
$$


Using Part (iii) of Property 5 in Assumption 2.3, we obtain

$$
\begin{aligned}
\left\langle w^{\prime}\right. & \left., \phi_{W}^{i ; p}\left(z_{1}\right) \psi_{W}^{a ; p}\left(z_{2}\right) v\right\rangle \\
& =\left\langle w^{\prime}, e^{-l_{p}\left(z_{1}-z_{2}\right) \mathcal{N}_{g}} e^{l_{p}\left(z_{1}-z_{2}\right) \mathcal{N}_{g}} \phi_{W}^{i ; p}\left(z_{1}\right) e^{-l_{p}\left(z_{1}-z_{2}\right) \mathcal{N}_{g}} e^{l_{p}\left(z_{1}-z_{2}\right) \mathcal{N}_{g}} \psi_{W}^{a ; p}\left(z_{2}\right) v\right\rangle \\
& \left.=\sum_{s \in \mathbb{N}} \frac{(-1)^{s}}{s !}\left(l_{p}\left(z_{1}-z_{2}\right)\right)^{s}\left\langle w^{\prime},[\overbrace{\mathcal{N}_{g}, \cdots,\left[\mathcal{N}_{g}\right.}^{s} e^{l_{p}\left(z_{1}-z_{2}\right) \mathcal{N}_{g}} \phi_{W}^{i ; p}\left(z_{1}\right) e^{-l_{p}\left(z_{1}-z_{2}\right) \mathcal{N}_{g}}] \cdots\right] \psi_{W}^{a ; p}\left(z_{2}\right) v\right\rangle \\
& \left.=\sum_{s \in \mathbb{N}} \frac{(-1)^{s}}{s !}\left(l_{p}\left(z_{1}-z_{2}\right)\right)^{s}\left\langle w^{\prime}, e^{l_{p}\left(z_{1}-z_{2}\right) \mathcal{N}_{g}}[\overbrace{\mathcal{N}_{g}, \cdots,\left[\mathcal{N}_{g}\right.}^{s}, \phi_{W}^{i ; p}\left(z_{1}\right)] \cdots\right] e^{-l_{p}\left(z_{1}-z_{2}\right) \mathcal{N}_{g}} \psi_{W}^{a ; p}\left(z_{2}\right) v\right\rangle \\
& =\sum_{s=0}^{N} \frac{(-1)^{s}}{s !}\left(l_{p}\left(z_{1}-z_{2}\right)\right)^{s}\left\langle w^{\prime}, e^{l_{p}\left(z_{1}-z_{2}\right) \mathcal{N}_{g}} \phi_{W}^{\mathcal{N}_{g}^{s}(i) ; p}\left(z_{1}\right) e^{-l_{p}\left(z_{1}-z_{2}\right) \mathcal{N}_{g}} \psi_{W}^{a ; p}\left(z_{2}\right) v\right\rangle .
\end{aligned}
$$

By (3.16), the right-hand side of (3.17) is absolutely convergent in the region given by $\left|z_{1}\right|>\left|z_{2}\right|>0$ and $\left|\arg \left(z_{1}-z_{2}\right)-\arg z_{1}\right|<\frac{\pi}{2}$ to

$$
\sum_{j, s, l, q=0}^{N} a_{q j s l} z_{1}^{-t} e^{-\left(\alpha_{i}+m_{q}\right) l_{p}\left(z_{1}-z_{2}\right)} e^{n_{j} l_{p}\left(z_{2}\right)}\left(l_{p}\left(z_{1}-z_{2}\right)\right)^{s}\left(l_{p}\left(z_{2}\right)\right)^{l}
$$

for some $a_{q j s l} \in \mathbb{C}$.

On the other hand, by Part (iii) of Property 5 in Assumption 2.3 ,

$$
\begin{aligned}
(-1)^{\left|\phi^{i}\right|\left|\psi^{a}\right|}\left\langle w^{\prime}, \psi_{W}^{a ; p}\left(z_{2}\right) \phi_{W}^{i ; p}\left(z_{1}\right) v\right\rangle & =(-1)^{\left|\phi^{i}\right|\left|\psi^{a}\right|}\left\langle w^{\prime}, \psi_{W}^{a ; p}\left(z_{2}\right) e^{-\pi i \mathcal{N}_{g}} e^{-l_{p}\left(z_{2}-z_{1}\right) \mathcal{N}_{g}} e^{l_{p}\left(z_{2}-z_{1}\right) \mathcal{N}_{g}} e^{\pi i \mathcal{N}_{g} .}\right. \\
& \left.\cdot \phi_{W}^{i ; p}\left(z_{1}\right) e^{-\pi i \mathcal{N}_{g}} e^{-l_{p}\left(z_{2}-z_{1}\right) \mathcal{N}_{g}} e^{l_{p}\left(z_{2}-z_{1}\right) \mathcal{N}_{g}} e^{\pi i \mathcal{N}_{g}} v\right\rangle \\
= & \sum_{s \in \mathbb{N}} \frac{(-1)^{s}}{s !}\left(l_{p}\left(z_{2}-z_{1}\right)+\pi i\right)^{s}(-1)^{\left|\phi^{i}\right|\left|\psi^{a}\right| .} \\
& \left.\cdot\left\langle w^{\prime}, \psi_{W}^{a ; p}\left(z_{2}\right)[\overbrace{\mathcal{N}_{g}, \cdots,\left[\mathcal{N}_{g}\right.}, e^{l_{p}\left(z_{2}-z_{1}\right) \mathcal{N}_{g}} e^{\pi i \mathcal{N}_{g}} \phi_{W}^{i ; p}\left(z_{1}\right) e^{-\pi i \mathcal{N}_{g}} e^{-l_{p}\left(z_{2}-z_{1}\right) \mathcal{N}_{g}}] \cdots\right] v\right\rangle \\
= & \sum_{s \in \mathbb{N}} \frac{(-1)^{s}}{s !}\left(l_{p}\left(z_{2}-z_{1}\right)+\pi i\right)^{s}(-1)^{\left|\phi^{i}\right|\left|\psi^{a}\right| .} \\
& \left.\cdot\left\langle w^{\prime}, \psi_{W}^{a ; p}\left(z_{2}\right) e^{l_{p}\left(z_{2}-z_{1}\right) \mathcal{N}_{g}} e^{\pi i \mathcal{N}_{g}}[\overbrace{\mathcal{N}_{g}, \cdots,\left[\mathcal{N}_{g}\right.}, \phi_{W}^{i ; p}\left(z_{1}\right)] \cdots\right] e^{-\pi i \mathcal{N}_{g}} e^{-l_{p}\left(z_{2}-z_{1}\right) \mathcal{N}_{g}} v\right\rangle \\
= & \sum_{s=0}^{N} \frac{(-1)^{s}}{s !}\left(l_{p}\left(z_{2}-z_{1}\right)+\pi i\right)^{s} \cdot \\
& \cdot(-1)^{\left|\phi^{i} \| \psi^{a}\right|}\left\langle w^{\prime}, \psi_{W}^{a ; p}\left(z_{2}\right) e^{l_{p}\left(z_{2}-z_{1}\right) \mathcal{N}_{g}} e^{\pi i \mathcal{N}_{g}} \phi_{W}^{\mathcal{N}_{g}^{s}(i) ; p}\left(z_{1}\right) e^{-\pi i \mathcal{N}_{g}} e^{-l_{p}\left(z_{2}-z_{1}\right) \mathcal{N}_{g}} v\right\rangle . \quad(3.19)
\end{aligned}
$$


By (3.16),

$$
(-1)^{\left|\phi^{i}\right|\left|\psi^{a}\right|}\left\langle w^{\prime}, \psi_{W}^{a ; p}\left(z_{2}\right) e^{l_{p}\left(z_{2}-z_{1}\right) \mathcal{N}_{g}} e^{\pi i \mathcal{N}_{g}} \phi_{W}^{\mathcal{N}_{g}^{s}(i) ; p}\left(z_{1}\right) e^{-\pi i \mathcal{N}_{g}} e^{-l_{p}\left(z_{2}-z_{1}\right) \mathcal{N}_{g}} v\right\rangle
$$

is absolutely convergent in the region given by $\left|z_{2}\right|>\left|z_{1}\right|>0$ and $\left|\arg \left(z_{1}-z_{2}\right)-\arg z_{2}\right|<\frac{\pi}{2}$ to

$$
F^{p}\left(\left\langle w^{\prime},\left(z_{1}-z_{2}\right)^{\mathcal{N}_{g}} \phi_{W}^{\mathcal{N}_{g}^{s}(i) ; p}\left(z_{1}\right)\left(z_{1}-z_{2}\right)^{-\mathcal{N}_{g}} \psi_{W}^{a}\left(z_{2}\right) v\right\rangle\right) .
$$

Thus the right-hand side of (3.19) is absolutely convergent in the region given by $\left|z_{2}\right|>$ $\left|z_{1}\right|>0$ and $\left|\arg \left(z_{1}-z_{2}\right)-\arg z_{2}\right|<\frac{\pi}{2}$ to (3.18). In particular, we have proved Property 6 in the case $k=1$ and $l=0$ and also (3.4). By Proposition 3.1, (3.1) also holds. Thus Property 16 holds.

We still need to prove Property 15 for general $k$ and $l$. For $i, j \in I$,

$$
\begin{aligned}
& \left(x_{1}-x\right)^{\mathcal{N}_{g}} \phi_{W}^{i}\left(x_{1}\right)\left(x_{1}-x\right)^{-\mathcal{N}_{g}}\left(x_{2}-x\right)^{\mathcal{N}_{g}} \phi_{W}^{j}\left(x_{2}\right)\left(x_{2}-x\right)^{-\mathcal{N}_{g}} \\
& \quad=\sum_{r, s \in \mathbb{N}} \frac{(-1)^{r+s}}{r ! s !}\left(\log \left(x_{1}-x\right)\right)^{r}\left(\log \left(x_{2}-x\right)\right)^{s} \phi_{W}^{\mathcal{N}_{g}^{r}(i)}\left(x_{1}\right) \phi_{W}^{\mathcal{N}_{g}^{s}(i)}\left(x_{2}\right)
\end{aligned}
$$

Since the right-hand side of (3.20) is in fact a finite sum, there exists $N_{i j} \in \mathbb{Z}_{+}$such that

$$
\begin{aligned}
& \left(x_{1}-x_{2}\right)^{N_{i j}}\left(x_{1}-x\right)^{\mathcal{N}_{g}} \phi_{W}^{i}\left(x_{1}\right)\left(x_{1}-x\right)^{-\mathcal{N}_{g}}\left(x_{2}-x\right)^{\mathcal{N}_{g}} \phi_{W}^{j}\left(x_{2}\right)\left(x_{2}-x\right)^{-\mathcal{N}_{g}} \\
& =\sum_{r, s \in \mathbb{N}} \frac{(-1)^{r+s}}{r ! s !}\left(\log \left(x_{1}-x\right)\right)^{r}\left(\log \left(x_{2}-x\right)\right)^{s}\left(x_{1}-x_{2}\right)^{N_{i j}} \phi_{W}^{\mathcal{N}_{g}^{r}(i)}\left(x_{1}\right) \phi_{W}^{\mathcal{N}_{g}^{s}(j)}\left(x_{2}\right) \\
& =\sum_{r, s \in \mathbb{N}} \frac{(-1)^{r+s}}{r ! s !}\left(\log \left(x_{1}-x\right)\right)^{r}\left(\log \left(x_{2}-x\right)\right)^{s} . \\
& \cdot(-1)^{\left|\phi^{\mathcal{N}_{g}^{r}(i)}\right|\left|\phi^{\mathcal{N}_{g}^{s}(i)}\right|}\left(x_{1}-x_{2}\right)^{N_{i j}} \phi_{W}^{\mathcal{N}_{g}^{s}(j)}\left(x_{2}\right) \phi_{W}^{\mathcal{N}_{g}^{r}(i)}\left(x_{1}\right) \\
& =\left(x_{1}-x_{2}\right)^{N_{i j}}\left(x_{2}-x\right)^{\mathcal{N}_{g}} \phi_{W}^{j}\left(x_{2}\right)\left(x_{2}-x\right)^{-\mathcal{N}_{g}}\left(x_{1}-x\right)^{\mathcal{N}_{g}} \phi_{W}^{i}\left(x_{1}\right)\left(x_{1}-x\right)^{-\mathcal{N}_{g}} .
\end{aligned}
$$

For general $k, l \in \mathbb{N}$, consider the series

$$
\begin{gathered}
\prod_{q=1}^{k+l}\left(x_{q}-x\right)^{\alpha_{i q}+M_{i_{q} a}} \prod_{m=1}^{l}\left(x-x_{k+m}\right)^{\alpha_{i_{k+m}}+M_{i_{k+m}}{ }^{a}} e^{\pi i\left(\alpha_{i_{k+m}}+M_{i_{k+m}}\right)} . \\
\cdot\left\langle w^{\prime},\left(x_{1}-x\right)^{\mathcal{N}_{g}} \phi_{W}^{i_{1}}\left(x_{1}\right)\left(x_{1}-x\right)^{-\mathcal{N}_{g}} \cdots\left(x_{k}-x\right)^{\mathcal{N}_{g}} \phi_{W}^{i_{k}}\left(x_{k}\right)\left(x_{k}-x\right)^{-\mathcal{N}_{g}} .\right. \\
\cdot \psi_{W}^{a}(x)\left(x-x_{k+1}\right)^{\mathcal{N}_{g}} e^{\pi i \mathcal{N}_{g}} \phi_{W}^{i_{k+1}}\left(x_{k+1}\right) e^{-\pi i \mathcal{N}_{g}}\left(x-x_{k+1}\right)^{-\mathcal{N}_{g} .} \\
\left.\cdots\left(x-x_{k+l}\right)^{\mathcal{N}_{g}} e^{\pi i \mathcal{N}_{g}} \phi_{W}^{i_{k+l}}\left(x_{k+l}\right) e^{-\pi i \mathcal{N}_{g}}\left(x-x_{k+l}\right)^{-\mathcal{N}_{g}} v\right\rangle,
\end{gathered}
$$

where $v \in V$ and $w^{\prime} \in W^{\prime}$. Using (2.2), we see that (3.22) is equal to

$$
\begin{aligned}
& \prod_{q=1}^{k+l}\left(x_{q}-x\right)^{\alpha_{i_{q}}+M_{i_{q} a}} \prod_{m=1}^{l}\left(x_{k+m}-x\right)^{\alpha_{i_{k+m}}+M_{i_{k+m}}} \cdot \\
& \quad \cdot\left\langle w^{\prime},\left(x_{1}-x\right)^{\mathcal{N}_{g}} \phi_{W}^{i_{1}}\left(x_{1}\right)\left(x_{1}-x\right)^{-\mathcal{N}_{g}} \cdots\left(x_{k}-x\right)^{\mathcal{N}_{g}} \phi_{W}^{i_{k}}\left(x_{k}\right) .\right.
\end{aligned}
$$




$$
\begin{aligned}
& \cdot\left(x_{k}-x\right)^{-\mathcal{N}_{g}}\left(x_{k+1}-x\right)^{\mathcal{N}_{g}} \phi_{W}^{i_{k+1}}\left(x_{k+1}\right)\left(x_{k+1}-x\right)^{-\mathcal{N}_{g}} . \\
& \left.\cdots\left(x_{k+l}-x\right)^{\mathcal{N}_{g}} \phi_{W}^{i_{k+l}}\left(x_{k+l}\right)\left(x_{k+l}-x\right)^{-\mathcal{N}_{g}} \psi_{W}^{a}(x) v\right\rangle,
\end{aligned}
$$

By (c) in Data 2.2, (3.23) has only finitely many negative integral powers in $x$ and finitely many nonnegative integral power terms in $\log x$. Using (2.2) again, we see that (3.22) is also equal to

$$
\begin{gathered}
\prod_{q=1}^{k+l}\left(x-x_{q}\right)^{\alpha_{i_{q}}+M_{i_{q} a} a} e^{\pi i\left(\alpha_{i_{q}}+M_{i_{q} a}\right)} \prod_{m=1}^{l}\left(x-x_{k+m}\right)^{\alpha_{i_{k+m}}+M_{i_{k+m} a}} e^{\pi i\left(\alpha_{i_{k+m}}+M_{i_{k+m} a}\right)} . \\
\cdot\left\langle w^{\prime}, \psi_{W}^{a}(x)\left(x-x_{1}\right)^{\mathcal{N}_{g}} e^{\pi i \mathcal{N}_{g}} \phi_{W}^{i_{1}}\left(x_{1}\right) e^{-\pi i \mathcal{N}_{g}}\left(x-x_{1}\right)^{-\mathcal{N}_{g}}\right. \\
\cdots\left(x-x_{k}\right)^{\mathcal{N}_{g}} e^{\pi i \mathcal{N}_{g}} \phi_{W}^{i_{k}}\left(x_{k}\right) e^{-\pi i \mathcal{N}_{g}}\left(x-x_{k}\right)^{-\mathcal{N}_{g}} \\
\cdot\left(x-x_{k+1}\right)^{\mathcal{N}_{g}} e^{\pi i \mathcal{N}_{g}} \phi_{W}^{i_{k+1}}\left(x_{k+1}\right) e^{-\pi i \mathcal{N}_{g}}\left(x-x_{k+1}\right)^{-\mathcal{N}_{g}} \\
\left.\cdots\left(x-x_{k+l}\right)^{\mathcal{N}_{g}} e^{\pi i \mathcal{N}_{g}} \phi_{W}^{i_{k+l}}\left(x_{k+l}\right) e^{-\pi i \mathcal{N}_{g}}\left(x-x_{k+l}\right)^{-\mathcal{N}_{g}} v\right\rangle
\end{gathered}
$$

By (c) in Data 2.2 and Property 12 in Proposition 2.4, (3.24) has only finitely many positive integral power terms in $x$ and finitely many nonnegative integral power terms in $\log x$. Thus (3.22), (3.23) and (3.24) are a Laurent polynomial in $x$ and a polynomial in $\log x$. In particular,

$$
\begin{gathered}
\left\langle w^{\prime},\left(x_{1}-x\right)^{\mathcal{N}_{g}} \phi_{W}^{i_{1}}\left(x_{1}\right)\left(x_{1}-x\right)^{-\mathcal{N}_{g}} \cdots\left(x_{k}-x\right)^{\mathcal{N}_{g}} \phi_{W}^{i_{k}}\left(x_{k}\right)\right. \\
\cdot\left(x_{k}-x\right)^{-\mathcal{N}_{g}}\left(x_{k+1}-x\right)^{\mathcal{N}_{g}} \phi_{W}^{i_{k+1}}\left(x_{k+1}\right)\left(x_{k+1}-x\right)^{-\mathcal{N}_{g}} \\
\left.\cdots\left(x_{k+l}-x\right)^{\mathcal{N}_{g}} \phi_{W}^{i_{k+l}}\left(x_{k+l}\right)\left(x_{k+l}-x\right)^{-\mathcal{N}_{g}} \psi_{W}^{a}(x) v\right\rangle
\end{gathered}
$$

is equal to this polynomial in $x, x^{-1}$ and $\log x$ with series in $x_{1}, \ldots, x_{k+l}$ as coefficients multiplied by

$$
\prod_{q=1}^{k+l}\left(x_{q}-x\right)^{-\left(\alpha_{i_{q}}+M_{i_{q} a}\right)} \prod_{m=1}^{l}\left(x-x_{k+m}\right)^{-\left(\alpha_{i_{k+m}}+M_{i_{k+m}}\right)} e^{-\pi i\left(\alpha_{i_{k+m}}+M_{i_{k+m} a}\right)} .
$$

The coefficients of this polynomial in $x, x^{-1}$ and $\log x$ are given by the coefficients of (3.23) in powers of $x$ and $\log x$. These coefficients are finite sums of products of (finite) linear combinations of powers of $x_{1}, \ldots, x_{k+l}, \log x_{1}, \ldots, \log x_{k+l}$ and series of the form

$$
\left\langle w^{\prime}, \phi_{W}^{\mathcal{N}_{g}^{j_{1}}\left(i_{1}\right)}\left(x_{1}\right) \cdots \phi_{W}^{\mathcal{N}_{g}^{j_{k+l}}\left(i_{k+l}\right)}\left(x_{k+l}\right)\left(\psi_{W}^{a}\right)_{r, s} v\right\rangle
$$

for $j_{1}, \ldots, j_{k+l} \in \mathbb{N}, r \in \mathbb{C}$ and $s \in \mathbb{N}$. By Property 13 in Theorem 3.1, we see that these coefficients with $x_{q}^{n}$ and $\log x_{q}$ substituted by $e^{n l_{p}\left(z_{q}\right)}$ and $l_{p}\left(z_{q}\right)$, respectively, are absolutely convergent in the region $\left|z_{1}\right|>\cdots>\left|z_{k+l}\right|>0$ to analytic functions of the form in Property 13 in Theorem 3.1 with $k$ there replaced by $k+l$. Thus (3.25) with $x_{q}^{n}, x^{n}, \log x_{q}$ and $\log x$ substituted by $e^{n l_{p}\left(z_{q}\right)}, e^{n l_{p}(z)}, l_{p}\left(z_{q}\right)$ and $l_{p}(z)$, respectively, is absolutely convergent in the 
region $\left|z_{1}\right|>\cdots>\left|z_{k}\right|>|z|>\left|z_{k+1}\right|>\cdots>\left|z_{k+l}\right|>0$ to an analytic function of the form (3.3), except that there are no factors of the forms $\left(l_{p}\left(z_{1}-z\right)\right)^{n_{1}}, \ldots,\left(l_{p}\left(z_{k+l}-z\right)\right)^{n_{k+l}}$. But

$$
\left\langle w^{\prime}, \phi_{W}^{i_{1} ; p}\left(z_{1}\right) \cdots \phi_{W}^{i_{k} ; p}\left(z_{k}\right) \psi_{W}^{a ; p}(z) \phi_{W}^{i_{k+1} ; p}\left(z_{k+1}\right) \cdots \phi_{W}^{i_{k+l} ; p}\left(z_{k+l}\right)\right\rangle
$$

is a linear combination of series of the form

$$
\begin{gathered}
\left\langle w^{\prime}, e^{l_{p}\left(z_{1}-z\right) \mathcal{N}_{g}} \phi_{W}^{i_{1} ; p}\left(z_{1}\right) e^{-l_{p}\left(z_{1}-z\right) \mathcal{N}_{g}} \cdots e^{l_{p}\left(z_{k}-z\right) \mathcal{N}_{g}} \phi_{W}^{i_{k} ; p}\left(z_{k}\right) e^{l_{p}\left(z_{k}-z\right) \mathcal{N}_{g}}\right. \\
\cdot \psi_{W}^{a ; p}(z) e^{l_{p}\left(z-z_{k+1} \mathcal{N}_{g}\right.} e^{\pi i \mathcal{N}_{g}} \phi_{W}^{i_{k+1} ; p}\left(z_{k+1}\right) e^{-\pi i \mathcal{N}_{g}} e^{-l_{p}\left(z-z_{k+1} \mathcal{N}_{g}\right.} \\
\left.\cdots e^{l_{p}\left(z-z_{k+l} \mathcal{N}_{g}\right.} e^{\pi i \mathcal{N}_{g}} \phi_{W}^{i_{k+l} ; p}\left(z_{k+l}\right) e^{-\pi i \mathcal{N}_{g}} e^{-l_{p}\left(z-z_{k+l} \mathcal{N}_{g}\right.} v\right\rangle
\end{gathered}
$$

with nonnegative powers of $l_{p}\left(z_{1}-z\right), \ldots, l_{p}\left(z_{k+l}-z\right)$ as coefficients. Thus (3.26) is absolutely convergent in the region given by $\left|z_{1}\right|>\cdots>\left|z_{k}\right|>|z|>\left|z_{k+1}\right|>\cdots>\left|z_{k+l}\right|>0$, $\left|\arg \left(z_{i}-z\right)-\arg z_{i}\right|<\frac{\pi}{2}$ for $i=1, \ldots, k$ and $\left|\arg \left(z_{i}-z\right)-\arg z\right|<\frac{\pi}{2}$ for $i=k+1, \ldots, k+l$ to an analytic function of the form (3.3). The remaining parts of Property 6 in Proposition 2.3 follows immediately from the proof above.

We have the following most general commutativity which follows immediately from Property 15 in Theorem 3.2. Property 14 in Theorem 3.1 and Property 16 in Theorem 3.2 .

Corollary 3.3 Assume that Properties 17 in Assumption 2.3 hold. Then for $v \in V, w^{\prime} \in$ $V^{\prime}$ and $\sigma \in S_{k}$,

$$
F^{p}\left(\left\langle w^{\prime}, \varphi_{1}\left(z_{1}\right) \cdots \varphi_{k}\left(z_{k}\right) v\right\rangle\right)= \pm F^{p}\left(\left\langle w^{\prime}, \varphi_{\sigma(1)}\left(z_{\sigma(1)}\right) \cdots \varphi_{\sigma(k)}\left(z_{\sigma(k)}\right) v\right\rangle\right),
$$

where one of $\varphi_{l}$ for $l=1, \ldots, k$ is $\psi^{a ; p}$ for some $a \in A$ and the others are in $\left\{\phi^{i ; p} \mid i \in I\right\}$ and the sign $\pm i$ s uniquely determined by $\sigma$ and $\left|\varphi_{1}\right|, \ldots,\left|\varphi_{k}\right|$.

\section{A construction theorem}

In this section, we construct a $g$-twisted $V$-module from the data in Data 2.2 satisfying Assumption 2.3.

First we need to define a twisted vertex operator map. Since we shall define the branches labeled by $p \in \mathbb{Z}$ of the twisted vertex operator map instead of defining the formal variable twisted vertex operator map, we need to show that these branches labeled by $p \in \mathbb{Z}$ determine a formal variable map uniquely. So we first prove the following lemma:

Lemma 4.1 Let $\phi$ be a multivalued analytic map with preferred branch $\phi^{0}$ from $\mathbb{C}^{\times}$to $\operatorname{Hom}(W, \bar{W})$ and $\phi^{p}$ for $p \in \mathbb{Z}$ are labeled branches of $\phi$. If there exists wt $\phi \in \frac{1}{2} \mathbb{Z}$ such that

$$
\left[L_{W}^{g}(0), \phi^{p}(z)\right]=z \frac{d}{d z} \phi^{p}(z)+(\operatorname{wt} \phi) \phi^{p}(z)
$$


then we have an expansion

$$
\phi^{p}(z)=\sum_{n \in \mathbb{C}} \sum_{k \in \mathbb{N}} \phi_{n, k} e^{(-n-1) l_{p}(z)}\left(l_{p}(z)\right)^{k}
$$

where $\phi_{n, k} \in \operatorname{Hom}(W, W)$ for $n \in \mathbb{C}$ and $k \in \mathbb{N}$ is homogeneous of weight wt $\phi-n-1$. In particular, we obtain a formal series

$$
\phi(x)=\sum_{n \in \mathbb{C}} \sum_{k \in \mathbb{N}} \phi_{n, k} x^{-n-1}(\log x)^{k} \in W\{x\}[\log x] .
$$

Moreover, for $w \in W$, there exist $N_{w} \in \mathbb{R}$ and $K_{n, w} \in \mathbb{N}$ for $n \in \mathbb{C}$ such that

$$
\phi^{p}(z) w=\sum_{\Re(n) \geq N_{w}} \sum_{k=0}^{K_{n, w}} \phi_{n, k} w e^{(-n-1) l_{p}(z)}\left(l_{p}(z)\right)^{k} .
$$

and for $w^{\prime} \in w^{\prime}$, there exist $N_{w^{\prime}} \in \mathbb{R}$ such that

$$
\left\langle w^{\prime}, \phi(z) \cdot\right\rangle=\sum_{\Re(n) \leq N_{w^{\prime}}} \sum_{k \in \mathbb{N}}\left\langle w^{\prime}, \phi_{n, k} \cdot\right\rangle e^{(-n-1) l_{p}(z)}\left(l_{p}(z)\right)^{k}
$$

Proof. First by Property 1 in Assumption 2.3, $L_{W}(0)$ can be decomposed as the sum of its semisimple part $L_{W}(0)_{S}$ and its nilpotent part $L_{W}(0)_{N}$. From the commutator formula for $L_{W}(0)$ and $\phi^{p}(z)$, we see that for $c \in \mathbb{C}$,

$$
e^{c L_{W}^{g}(0)} \phi^{p}(z) e^{-c L_{W}^{g}(0)}=e^{c(\operatorname{wt} \phi)} \phi^{p}\left(e^{c} z\right)
$$

In particular, taking $c=-l_{p}(z)$, we obtain

$$
\begin{aligned}
\phi^{p}(z) & =e^{-l_{p}(z)(\operatorname{wt} \phi)} e^{l_{p}(z) L_{W}^{g}(0)} \phi^{p}(1) e^{-l_{p}(z) L_{W}^{g}(0)} \\
& =e^{-l_{p}(z)(\operatorname{wt} \phi)} e^{l_{p}(z) L_{W}^{g}(0)_{S}} e^{l_{p}(z) L_{W}^{g}(0)_{N}} \phi^{p}(1) e^{-l_{p}(z) L_{W}^{g}(0)_{N}} e^{-l_{p}(z) L_{W}^{g}(0)_{S}} .
\end{aligned}
$$

For $n \in \mathbb{C}$ and $w \in W_{[m]}$,

$$
e^{l_{p}(z) L_{W}^{g}(0)_{N}} \pi_{(\operatorname{wt} \phi)-n-1+m} \phi^{p}(1) e^{-l_{p}(z) L_{W}^{g}(0)_{N}} w
$$

is in fact a polynomial in $l_{p}(z)$ with coefficients in $W_{[(\operatorname{wt} \phi)-n-1+m]}$, where for $r \in \mathbb{C}$, we use $\pi_{r}$ to denote the projection from $W$ to $W_{[r]}$. Let $K_{n, w}$ be the degree of this polynomial in $l_{p}(z)$ and let $\phi_{n, k} w \in W$ for $k \in \mathbb{N}$ be the coefficient of the $k$-th power of $l_{p}(z)$ in

$$
e^{l_{p}(z) L_{W}^{g}(0)_{N}} \pi_{(\operatorname{wt} \phi)-n-1+m} \phi^{p}(1) e^{-l_{p}(z) L_{W}^{g}(0)_{N}} w .
$$

We obtain $\phi_{n, k} \in \operatorname{Hom}(W, W)$ of weight wt $\phi-n-1$ such that

$$
\sum_{k=0}^{K_{n, w}} \phi_{n, k} w\left(l_{p}(z)\right)^{k}=e^{l_{p}(z) L_{W}^{g}(0)_{N}} \pi_{(\operatorname{wt} \phi)-n-1+m} \phi^{p}(1) e^{-l_{p}(z) L_{W}^{g}(0)_{N}} w .
$$


Then for $n \in \mathbb{Z}$ and $w \in W$,

$$
\begin{aligned}
& e^{-l_{p}(z)(\mathrm{wt} \phi)} e^{l_{p}(z) L_{W}^{g}(0)_{S}} \sum_{k=0}^{K_{n, w}} \phi_{n, k} e^{-l_{p}(z) L_{W}^{g}(0)_{S}} w\left(l_{p}(z)\right)^{k} \\
& \quad=e^{l_{p}(z) L_{W}^{g}(0)_{S}} e^{l_{p}(z) L_{W}^{g}(0)_{N}} \pi_{(\mathrm{wt} \phi)-n-1+m} \phi^{p}(1) e^{-l_{p}(z) L_{W}^{g}(0)_{N}} e^{-l_{p}(z) L_{W}^{g}(0)_{S}} w .
\end{aligned}
$$

Thus for $w \in W$,

$$
\begin{aligned}
\phi^{p}(z) w & =e^{-l_{p}(z)(\operatorname{wt} \phi)} e^{l_{p}(z) L_{W}^{g}(0)_{S}} e^{l_{p}(z) L_{W}^{g}(0)_{N}} \phi^{p}(1) e^{-l_{p}(z) L_{W}^{g}(0)_{N}} e^{-l_{p}(z) L_{W}^{g}(0)_{S}} w \\
& =\sum_{n \in \mathbb{C}} e^{-l_{p}(z)(\operatorname{wt} \phi)} e^{l_{p}(z) L_{W}^{g}(0)_{S}} e^{l_{p}(z) L_{W}^{g}(0)_{N}} \pi_{(\operatorname{wt} \phi)-n-1+m} \phi^{p}(1) e^{-l_{p}(z) L_{W}^{g}(0)_{N}} e^{-l_{p}(z) L_{W}^{g}(0)_{S}} w \\
& =\sum_{n \in \mathbb{C}} \sum_{k=0}^{K_{n, w}} e^{-l_{p}(z)(\operatorname{wt} \phi)} e^{-l_{p}(z)(\operatorname{wt} \phi)} e^{l_{p}(z) L_{W}^{g}(0)_{S}} \phi_{n, k} e^{-l_{p}(z) L_{W}^{g}(0)_{S}} w\left(l_{p}(z)\right)^{k} \\
& =\sum_{n \in \mathbb{Z}} \sum_{k=0}^{K_{n, w}} \phi_{n, k} w e^{(-n-1) l_{p}(z)}\left(l_{p}(z)\right)^{k} .
\end{aligned}
$$

Since $W$ is lower bounded with respect to the weights and the weight of $\phi_{n, k}$ is wt $\phi-n-1$, we obtain (4.2) from (4.4) and we also have (4.3). Since nonhomogeneous elements of $W$ are finite sums of homogeneous elements of $W$, (4.1), (4.2) and (4.3) also holds for general $w \in W$.

The vertex operator map we want to define is a linear map

$$
\begin{aligned}
Y_{W}^{g}: V \otimes W & \rightarrow W\{x\}[\log x], \\
u \otimes w & \mapsto Y_{W}^{g}(u, x) w .
\end{aligned}
$$

Such a map gives a multivalued analytic map (denoted using the same notation)

$$
\begin{aligned}
Y_{W}^{g}: \mathbb{C}^{\times} & \rightarrow \operatorname{Hom}(V \otimes W, \bar{W}), \\
z & \mapsto Y_{W}^{g}(\cdot, z) \cdot: u \otimes w \mapsto Y_{W}^{g}(u, z) w
\end{aligned}
$$

with labeled branches

$$
\begin{aligned}
\left(Y_{W}^{g}\right)^{p}: \mathbb{C}^{\times} & \rightarrow \operatorname{Hom}(V \otimes W, \bar{W}), \\
z & \mapsto\left(Y_{W}^{g}\right)^{p}(\cdot, z) \cdot: u \otimes w \mapsto\left(Y_{W}^{g}\right)^{p}(u, z) w
\end{aligned}
$$

for $p \in \mathbb{Z}$. Conversely, by Lemma 4.1 such a multivalued analytic map with labeled branches also determines a linear map of the form (4.5). Thus to define a twisted vertex operator map, we need only define $\left(Y_{W}^{g}\right)^{p}$.

We first give the motivation of our definition. The idea is in fact the same as in [H2]. We define $\left(Y_{W}^{g}\right)^{p}\left(\phi_{-1}^{i} \mathbf{1}, z\right) w=\phi_{W}^{i ; p}(z) w$ for $p \in \mathbb{Z}, i \in I$ and $w \in W$. The vertex operator map should satisfy the duality property. In particular, we should have

$$
F^{p}\left(\left\langle w^{\prime}, Y_{W}^{g}\left(\phi^{i_{1}}\left(\xi_{1}\right) \cdots \phi^{i_{k}}\left(\xi_{k}\right) \mathbf{1}, z\right) w\right\rangle\right)=F^{p}\left(\left\langle w^{\prime}, \phi_{W}^{i_{1}}\left(\xi_{1}+z\right) \cdots \phi_{W}^{i_{k}}\left(\xi_{k}+z\right) w\right\rangle\right)
$$


for $i_{1}, \ldots, i_{k} \in I, w \in W$ and $w^{\prime} \in W^{\prime}$. Note that $\phi^{i_{1}}\left(\xi_{1}\right), \ldots, \phi^{i_{k}}\left(\xi_{k}\right)$ are single-valued analytic functions in $\xi_{1}, \ldots, \xi_{k}$, respectively. Also by Property 13 in Theorem 3.1, the righthand side of (4.6) is a single-valued analytic function of $\xi_{1}, \ldots, \xi_{k}$ when $\xi_{i}+z \neq 0$ for $i=1, \ldots, k, \xi_{i} \neq \xi_{j}$ for $i, j=1, \ldots, k$ and $i \neq j$.

Motivated by (4.6), we define the vertex operator map as follows: For $w^{\prime} \in W^{\prime}, w \in W$, $i_{1}, \ldots, i_{k} \in I, m_{1}, \ldots, m_{k} \in \mathbb{Z}$, we define $\left(Y_{W}^{g}\right)^{p}$ by

$$
\begin{aligned}
& \left\langle w^{\prime},\left(Y_{W}^{g}\right)^{p}\left(\phi_{m_{1}}^{i_{1}} \cdots \phi_{m_{k}}^{i_{k}} \mathbf{1}, z\right) w\right\rangle \\
& \quad=\operatorname{Res}_{\xi_{1}=0} \cdots \operatorname{Res}_{\xi_{k}=0} \xi_{1}^{m_{1}} \cdots \xi_{k}^{m_{k}} F^{p}\left(\left\langle w^{\prime}, \phi_{W}^{i_{1}}\left(\xi_{1}+z\right) \cdots \phi_{W}^{i_{k}}\left(\xi_{k}+z\right) w\right\rangle\right) .
\end{aligned}
$$

Since there might be relations among elements of the form $\phi_{m_{1}}^{i_{1}} \cdots \phi_{m_{k}}^{i_{k}} \mathbf{1}$, we first have to show that the definition above indeed gives a well-defined map from $\mathbb{C}^{\times}$to $\operatorname{Hom}(V \otimes W, \bar{W})$. Let $\phi^{0}$ be the map from $\mathbb{C}^{\times}$to $\operatorname{Hom}(V, \bar{V})$ given by $\phi^{0}(z)=1_{V}$. Let wt $\phi^{0}=0$. Then Properties 107 in Assumption 2.3 and Properties 812 in Proposition 2.4 still hold for $\phi^{i}$, $i \in \tilde{I}=I \cup\{0\}$. Then any relation among such elements can always be written as

$$
\sum_{\mu=1}^{M} \lambda_{\mu} \phi_{m_{1}^{\mu}}^{i_{1}^{\mu}} \cdots \phi_{m_{k}^{\mu}}^{i_{k}^{\mu}} \mathbf{1}=0
$$

for some $k \in \mathbb{Z}_{+}, i_{j}^{\mu} \in \tilde{I}$ and $m_{j}^{\mu} \in \mathbb{Z}$ for $\mu=1, \ldots, M, j=1, \ldots, k$, where $\phi_{m_{1}^{\mu}}^{i_{1}^{\mu}} \cdots \phi_{m_{k}^{\mu}}^{i_{k}^{\mu}} \mathbf{1}$ for $\mu=1, \ldots, M$ either all belong to $V^{0}$ or all belong to $V^{1}$, that is, $\left|\phi_{m_{1}^{\mu}}^{i_{1}^{\mu}}\right|+\cdots+\left|\phi_{m_{k}^{\mu}}^{i_{k}^{\mu}}\right|$ for $\mu=1, \ldots, M$ are either all even or are all odd. In particular, the parities of $\left|\phi_{m_{1}^{\mu}}^{i_{1}^{\mu}}\right|+\cdots+\left|\phi_{m_{k}^{\mu}}^{i_{i}^{\mu}}\right|$ are independent of $\mu$. Since the parity $\left|\phi_{m_{r}^{\mu}}^{i_{\mu}^{\mu}}\right|$ for $\mu=1, \ldots, M, r=1, \ldots, k$ are equal to the parity $\left|\phi^{i_{r}^{\mu}}\right|$, we see that the parities of $\left|\phi^{i_{1}^{r}}\right|+\cdots+\left|\phi^{i_{k}^{\mu}}\right|$ are independent of $\mu$.

Lemma 4.2 If

$$
\sum_{\mu=1}^{M} \lambda_{\mu} \phi_{m_{1}^{i_{1}^{\mu}}}^{i_{\mu}^{\mu}} \cdots \phi_{m_{k}^{\mu}}^{i_{k}^{\mu}} \mathbf{1}=0
$$

then

$$
\sum_{\mu=1}^{M} \lambda_{\mu} \operatorname{Res}_{\xi_{1}=0} \cdots \operatorname{Res}_{\xi_{k}=0} \xi_{1}^{m_{1}^{\mu}} \cdots \xi_{k}^{m_{k}^{\mu}} F^{p}\left(\left\langle w^{\prime}, \phi_{W}^{i_{1}^{\mu}}\left(\xi_{1}+z\right) \cdots \phi_{W}^{i_{k}^{\mu}}\left(\xi_{k}+z\right) w\right\rangle\right)=0
$$

for $w \in W$ and $w^{\prime} \in W^{\prime}$.

Proof. Since $V$ is generated by $\phi^{i}(x)$ for $i \in I$, by Property 4 in Assumption 2.3, we can take

$$
w=\left(\phi_{W}\right)_{n_{1}, q_{1}}^{j_{1}} \cdots\left(\phi_{W}\right)_{n_{l}, q_{l}}^{j_{l}}\left(\psi_{W}^{a}\right)_{n, q} \phi_{n_{l+1}}^{j_{l+1}} \cdots \phi_{n_{m}}^{j_{m}} \mathbf{1} .
$$

Since this element is a coefficient of

$$
\phi_{W}^{j_{1}}\left(\zeta_{1}\right) \cdots \phi_{W}^{j_{l}}\left(\zeta_{l}\right) \psi_{W}^{a}(\zeta) \phi^{j_{l+1}}\left(\zeta_{l+1}\right) \cdots \phi^{j_{m}}\left(\zeta_{m}\right) \mathbf{1},
$$


we first prove

$$
\begin{aligned}
& \sum_{\mu=1}^{M} \lambda_{\mu} \operatorname{Res}_{\xi_{1}=0} \cdots \operatorname{Res}_{\xi_{k}=0} \xi_{1}^{m_{1}^{\mu}} \cdots \xi_{k}^{m_{k}^{\mu}} \cdot \\
& \quad \cdot F^{p}\left(\left\langle w^{\prime}, \phi_{W}^{i_{1}^{\mu}}\left(\xi_{1}+z\right) \cdots \phi_{W}^{i_{k}^{\mu}}\left(\xi_{k}+z\right) \phi_{W}^{j_{1}}\left(\zeta_{1}\right) \cdots \phi_{W}^{j_{l}}\left(\zeta_{l}\right) \psi_{W}^{a}(\zeta) \phi^{j_{l+1}}\left(\zeta_{l+1}\right) \cdots \phi^{j_{m}}\left(\zeta_{m}\right) \mathbf{1}\right\rangle\right)=0 .
\end{aligned}
$$

We have

$$
\begin{aligned}
& \operatorname{Res}_{\xi_{1}=0} \cdots \operatorname{Res}_{\xi_{k}=0} \xi_{1}^{m_{1}^{\mu}} \cdots \xi_{k}^{m_{k}^{\mu}} . \\
& \cdot F^{p}\left(\left\langle w^{\prime}, \phi_{W}^{i_{1}^{\mu}}\left(\xi_{1}+z\right) \cdots \phi_{W}^{i_{k}^{\mu}}\left(\xi_{k}+z\right) \phi_{W}^{j_{1}}\left(\zeta_{1}\right) \cdots \phi_{W}^{j_{l}}\left(\zeta_{l}\right) \psi_{W}^{a}(\zeta) \phi^{j_{l+1}}\left(\zeta_{l+1}\right) \cdots \phi^{j_{m}}\left(\zeta_{m}\right) \mathbf{1}\right\rangle\right) \\
& =\prod_{r=1}^{k} \prod_{s=1}^{m}(-1)^{\left|\phi^{i_{r}^{\mu}}\right|\left|\phi^{j_{s}}\right|} \prod_{r=1}^{k}(-1)^{\left|\phi^{i_{r}^{\mu}} \| \psi_{W}^{a}\right|} \operatorname{Res}_{\xi_{1}=0} \cdots \operatorname{Res}_{\xi_{k}=0} \xi_{1}^{m_{1}^{\mu}} \cdots \xi_{k}^{m_{k}^{\mu}} \\
& \cdot F^{p}\left(\left\langle w^{\prime}, \phi_{W}^{j_{1}}\left(\zeta_{1}\right) \cdots \phi_{W}^{j_{l}}\left(\zeta_{l}\right) \psi_{W}^{a}(\zeta) \phi^{j_{l+1}}\left(\zeta_{l+1}\right) \cdots \phi^{j_{m}}\left(\zeta_{m}\right) \phi^{i_{1}^{\mu}}\left(\xi_{1}+z\right) \cdots \phi^{i_{k}^{\mu}}\left(\xi_{k}+z\right) \mathbf{1}\right\rangle\right) \\
& =\prod_{s=1}^{m}(-1)\left(\left|\phi^{i_{1}^{\mu}}\right|+\cdots+\left|\phi^{i_{k}^{\mu}}\right|\right)\left|\phi^{j_{s}}\right|(-1)\left(\left|\phi^{i_{1}^{\mu}}\right|+\cdots+\left|\phi^{i^{\mu}}\right|\right)\left|\psi_{W}^{a}\right| \operatorname{Res}_{\xi_{1}=0} \cdots \operatorname{Res}_{\xi_{k}=0} \xi_{1}^{m_{1}^{\mu}} \cdots \xi_{k}^{m_{k}^{\mu}} \text {. } \\
& \cdot F^{p}\left(\left\langlee^{z L_{W}(-1)^{\prime}} w^{\prime}, \phi_{W}^{j_{1}}\left(\zeta_{1}-z\right) \cdots \phi_{W}^{j_{l}}\left(\zeta_{l}-z\right) \psi_{W}^{a}(\zeta-z) \cdot\right.\right. \\
& \left.\left.\cdot \phi^{j_{l+1}}\left(\zeta_{l+1}-z\right) \cdots \phi^{j_{m}}\left(\zeta_{m}-z\right) \phi^{i_{1}^{\mu}}\left(\xi_{1}\right) \cdots \phi^{i_{k}^{\mu}}\left(\xi_{k}\right) \mathbf{1}\right\rangle\right) \\
& =\prod_{s=1}^{m}(-1)\left(\left|\phi^{i_{1}^{\mu}}\right|+\cdots+\left|\phi^{i}{ }_{k}^{\mu}\right|\right)\left|\phi^{j_{s}}\right|(-1)\left(\left|\phi^{i_{1}^{\mu}}\right|+\cdots+\left|\phi^{i^{i} k}\right|\right)\left|\psi_{W}^{a}\right| . \\
& \cdot F^{p}\left(\left\langlee^{z L_{W}(-1)^{\prime}} w^{\prime}, \phi_{W}^{j_{1}}\left(\zeta_{1}-z\right) \cdots \phi_{W}^{j_{l}}\left(\zeta_{l}-z\right) \psi_{W}^{a}(\zeta-z) \cdot\right.\right. \\
& \left.\left.\cdot \phi^{j_{l+1}}\left(\zeta_{l+1}-z\right) \cdots \phi^{j_{m}}\left(\zeta_{m}-z\right) \phi_{m_{1}^{\mu}}^{i_{1}^{p}} \cdots \phi_{m_{k}^{\mu}}^{i_{k}^{p}} \mathbf{1}\right\rangle\right) \text {. }
\end{aligned}
$$

Recalling that $\left|\phi^{i_{1}^{\mu}}\right|+\cdots+\left|\phi^{i_{k}^{\mu}}\right|$ are independent of $\mu$, we obtain

$$
\begin{aligned}
& \sum_{\mu=1}^{M} \lambda_{\mu} \operatorname{Res}_{\xi_{1}=0} \cdots \operatorname{Res}_{\xi_{k}=0} \xi_{1}^{m_{1}^{\mu}} \cdots \xi_{k}^{m_{k}^{\mu}} \\
& \cdot F^{p}\left(\left\langle w^{\prime}, \phi_{W}^{i_{1}^{\mu}}\left(\xi_{1}+z\right) \cdots \phi_{W}^{i_{k}^{\mu}}\left(\xi_{k}+z\right) \phi_{W}^{j_{1}}\left(\zeta_{1}\right) \cdots \phi_{W}^{j_{l}}\left(\zeta_{l}\right) \psi_{W}^{a}(\zeta) \phi^{j_{l+1}}\left(\zeta_{l+1}\right) \cdots \phi^{j_{m}}\left(\zeta_{m}\right) \mathbf{1}\right\rangle\right) \\
& =\sum_{\mu=1}^{M} \prod_{s=1}^{m}(-1)\left(\left|\phi^{i_{1}^{\mu}}\right|+\cdots+\left|\phi^{i_{k}^{\mu}}\right|\right)\left|\phi^{j_{s}}\right|(-1)\left(\left|\phi^{i_{1}^{\mu}}\right|+\cdots+\left|\phi^{i_{k}^{\mu}}\right|\right)\left|\psi_{W}^{a}\right| \text {. } \\
& \cdot F^{p}\left(\left\langlee^{z L_{W}(-1)^{\prime}} w^{\prime}, \phi_{W}^{j_{1}}\left(\zeta_{1}-z\right) \cdots \phi_{W}^{j_{l}}\left(\zeta_{l}-z\right) \psi_{W}^{a}(\zeta-z) \cdot\right.\right. \\
& \left.\left.\cdot \phi^{j_{l+1}}\left(\zeta_{l+1}-z\right) \cdots \phi^{j_{m}}\left(\zeta_{m}-z\right) \phi_{m_{1}^{\mu}}^{i_{1}^{p}} \cdots \phi_{m_{k}^{\mu}}^{i_{k}^{p}} \mathbf{1}\right\rangle\right) \\
& =\prod_{s=1}^{m}(-1)\left(\left|\phi^{i_{1}^{\mu}}\right|+\cdots+\left|\phi^{i_{k}^{\mu}}\right|\right)\left|\phi^{j_{s}}\right|(-1)\left(\mid \phi^{\left.i_{1}^{\mu}|+\cdots+| \phi^{i_{k}^{\mu}} \mid\right)\left|\psi_{W}^{a}\right|}\right. \text {. }
\end{aligned}
$$




$$
\begin{aligned}
& \cdot F^{p}\left(\left\langlee^{z L_{W}(-1)^{\prime}} w^{\prime}, \phi_{W}^{j_{1}}\left(\zeta_{1}-z\right) \cdots \phi_{W}^{j_{l}}\left(\zeta_{l}-z\right) \psi_{W}^{a}(\zeta-z) \cdot\right.\right. \\
& \left.\left.\cdot \phi^{j_{l+1}}\left(\zeta_{l+1}-z\right) \cdots \phi^{j_{m}}\left(\zeta_{m}-z\right)\left(\sum_{\mu=1}^{M} \lambda_{\mu} \phi_{m_{1}^{\mu}}^{i_{1}^{\mu}} \cdots \phi_{m_{k}^{\mu}}^{i_{k}^{\mu}} \mathbf{1}\right)\right\rangle\right) \\
& =0
\end{aligned}
$$

proving (4.9).

For any fixed $z, \xi_{1}, \ldots, \xi_{l}, \xi$, the left-hand side of (4.9) can be expanded in the region $|z|,\left|\xi_{1}\right|, \ldots,\left|\xi_{l}\right|,|\xi|>\left|\zeta_{l+1}\right|>\cdots>\left|\zeta_{m}\right|$ as

$$
\begin{aligned}
& \sum_{n_{1}, \ldots, n_{m} \in \mathbb{Z}} \sum_{\mu=1}^{M} \lambda_{\mu} \operatorname{Res}_{\xi_{1}=0} \cdots \operatorname{Res}_{\xi_{k}=0} \xi_{1}^{m_{1}^{\mu}} \cdots \xi_{k}^{m_{k}^{\mu}} \\
& \cdot F^{p}\left(\left\langle w^{\prime}, \phi_{W}^{i_{1}^{\mu}}\left(\xi_{1}+z\right) \cdots \phi_{W}^{i_{k}^{\mu}}\left(\xi_{k}+z\right) \phi_{W}^{j_{1}}\left(\zeta_{1}\right) \cdots \phi_{W}^{j_{l}}\left(\zeta_{l}\right) \psi_{W}^{a}(\xi) \phi_{n_{l+1}}^{j_{l+1}} \cdots \phi_{n_{m}}^{j_{m}} \mathbf{1}\right\rangle\right) . \\
& \cdot \zeta_{l+1}^{-n_{l+1}-1} \cdots \zeta_{m}^{-n_{m}-1} .
\end{aligned}
$$

By (4.9), the Laurent series (4.10) in $\zeta_{l+1}, \ldots, \zeta_{m}$ is also 0 and thus its coefficients are all 0 . So we obtain

$$
\begin{aligned}
\sum_{\mu=1}^{M} \lambda_{\mu} & \operatorname{Res}_{\xi_{1}=0} \cdots \operatorname{Res}_{\xi_{k}=0} \xi_{1}^{m_{1}^{\mu}} \cdots \xi_{k}^{m_{k}^{\mu}} \\
& \cdot F^{p}\left(\left\langle w^{\prime}, \phi_{W}^{i_{1}^{\mu}}\left(\xi_{1}+z\right) \cdots \phi_{W}^{i_{k}^{\mu}}\left(\xi_{k}+z\right) \phi_{W}^{j_{1}}\left(\zeta_{1}\right) \cdots \phi_{W}^{j_{l}}\left(\zeta_{l}\right) \psi_{W}^{a}(\xi) \phi_{n_{l+1}}^{j_{l+1}} \cdots \phi_{n_{m}}^{j_{m}} \mathbf{1}\right\rangle\right) \\
= & 0 .
\end{aligned}
$$

By Assumption 2.1, $\phi_{n_{l+1}}^{j_{l+1}} \cdots \phi_{n_{m}}^{j_{m}} \mathbf{1}$ is a generalized eigenvector for $g$ with the eigenvalue $e^{2 \pi i\left(\alpha^{j_{l+1}+\cdots+\alpha^{j m}}\right)}$. Then there exists $N, K \in \mathbb{N}$ such that the left-hand side of (4.11) can be expanded when $\xi$ is sufficiently small but not 0 as

$$
\begin{aligned}
& \sum_{q=0}^{K} \sum_{n \in \alpha^{j_{l}+1}+\cdots+\alpha^{j}+N-\mathbb{N}} \sum_{\mu=1}^{M} \lambda_{\mu} \operatorname{Res}_{\xi_{1}=0} \cdots \operatorname{Res}_{\xi_{k}=0} \xi_{1}^{m_{1}^{\mu}} \cdots \xi_{k}^{m_{k}^{\mu}} \\
& \cdot F^{p}\left(\left\langle w^{\prime}, \phi_{W}^{i_{1}^{\mu}}\left(\xi_{1}+z\right) \cdots \phi_{W}^{i_{k}^{\mu}}\left(\xi_{k}+z\right) \phi_{W}^{j_{1}}\left(\zeta_{1}\right) \cdots \phi_{W}^{j_{l}}\left(\zeta_{l}\right)\left(\psi_{W}^{a}\right)_{n, q} \phi_{n_{l+1}}^{j_{l+1}} \cdots \phi_{n_{m}}^{j_{m}} \mathbf{1}\right\rangle\right) \xi^{-n-1} l_{p}(\xi)^{q} .
\end{aligned}
$$

By (4.11) and the fact that $\left(-\alpha^{j_{l+1}}-\cdots-\alpha^{j_{m}}-N+\mathbb{N}\right) \times\{1, \ldots, K\}$ is a unique expansion set (see Proposition 2.1 in [H4]), the expansion coefficients of (4.12) must be 0, that is,

$$
\begin{aligned}
\sum_{\mu=1}^{M} \lambda_{\mu} & \operatorname{Res}_{\xi_{1}=0} \cdots \operatorname{Res}_{\xi_{k}=0} \xi_{1}^{m_{1}^{\mu}} \cdots \xi_{k}^{m_{k}^{\mu}} \\
& \cdot F^{p}\left(\left\langle w^{\prime}, \phi_{W}^{i_{1}^{\mu}}\left(\xi_{1}+z\right) \cdots \phi_{W}^{i_{k}^{\mu}}\left(\xi_{k}+z\right) \phi_{W}^{j_{1}}\left(\zeta_{1}\right) \cdots \phi_{W}^{j_{l}}\left(\zeta_{l}\right)\left(\psi_{W}^{a}\right)_{n, q} \phi_{n_{l+1}}^{j_{l+1}} \cdots \phi_{n_{m}}^{j_{m}} \mathbf{1}\right\rangle\right) \\
= & 0 .
\end{aligned}
$$


By Data2.2, there exists $N_{l}, K_{l} \in \mathbb{N}$ such that the left-hand side of (4.13) can be expanded when $\left|\zeta_{l}\right|$ is sufficiently small but not 0 as

$$
\begin{aligned}
& \sum_{q_{l}=0}^{K_{l}} \sum_{n_{l} \in \alpha^{j_{l}+N_{l}-\mathbb{N}}} \sum_{\mu=1}^{M} \lambda_{\mu} \operatorname{Res}_{\xi_{1}=0} \cdots \operatorname{Res}_{\xi_{k}=0} \xi_{1}^{m_{1}^{\mu}} \cdots \xi_{k}^{m_{k}^{\mu}} \cdot \\
& \quad \cdot F^{p}\left(\left\langle w^{\prime}, \phi_{W}^{i_{1}^{\mu}}\left(\xi_{1}+z\right) \cdots \phi_{W}^{i_{k}^{\mu}}\left(\xi_{k}+z\right) \phi_{W}^{j_{1}}\left(\zeta_{1}\right) \cdots \phi_{W}^{j_{l-1}}\left(\zeta_{l-1}\right)\left(\phi_{W}^{j_{l}}\right)_{n_{l}, q_{l}}\left(\psi_{W}^{a}\right)_{n, q} \phi_{n_{l+1}}^{j_{l+1}} \cdots \phi_{n_{m}}^{j_{m}} \mathbf{1}\right\rangle\right) \\
& \quad \cdot e^{\left(-n_{l}-1\right) l_{p}\left(\zeta_{l}\right)}\left(l_{p}\left(\zeta_{l}\right)\right)^{q_{l}} .
\end{aligned}
$$

By (4.13), (4.14) and the fact that $\left(-\alpha^{j_{l}}-N_{l}+\mathbb{N}\right) \times\left\{1, \ldots, K_{l}\right\}$ is a unique expansion set (again see Proposition 2.1 in [H4]), the expansion coefficients of (4.14) must be 0, that is,

$$
\begin{aligned}
& \sum_{\mu=1}^{M} \lambda_{\mu} \operatorname{Res}_{\xi_{1}=0} \cdots \operatorname{Res}_{\xi_{k}=0} \xi_{1}^{m_{1}^{\mu}} \cdots \xi_{k}^{m_{k}^{\mu}} \cdot \\
& \quad F^{p}\left(\left\langlew^{\prime}, \phi_{W}^{i_{1}^{\mu}}\left(\xi_{1}+z\right) \cdots \phi_{W}^{i_{k}^{\mu}}\left(\xi_{k}+z\right) \cdot\right.\right. \\
& \left.\left.\quad \cdot \phi_{W}^{j_{1}}\left(\zeta_{1}\right) \cdots \phi_{W}^{j_{l-1}}\left(\zeta_{l-1}\right)\left(\phi_{W}^{j_{l}}\right)_{n_{l}, q_{l}}\left(\psi_{W}^{a}\right)_{n, q} \phi_{n_{l+1}}^{j_{l+1}} \cdots \phi_{n_{m}}^{j_{m}} \mathbf{1}\right\rangle\right) \\
& \quad=0 .
\end{aligned}
$$

Continuing this process repeatedly for $\phi_{W}^{j_{l-1}}\left(\zeta_{l-1}\right), \ldots, \phi_{W}^{j_{1}}\left(\zeta_{1}\right)$, we obtain (4.8).

From this lemma, we see that $\left(Y_{W}^{g}\right)^{p}$ and thus the vertex operator map $Y_{W}^{g}$ are well defined.

The following result is our construction theorem:

Theorem 4.3 The pair $\left(W, Y_{W}^{g}\right)$ is a lower-bounded generalized $g$-twisted $V$-module generated by $\left(\psi_{W}^{a}\right)_{n, k} v$ for $a \in A, n \in \alpha+\mathbb{Z}, k \in \mathbb{N}, v \in V^{[\alpha]}$ and $\alpha \in P_{V}$. Moreover, this is the unique lower-bounded generalized $g$-twisted $V$-module structure on $W$ generated by $\left(\psi_{W}^{a}\right)_{n, k} v$ for $a \in A, n \in \alpha+\mathbb{Z}, k \in \mathbb{N}, v \in V^{[\alpha]}$ and $\alpha \in P_{V}$ such that $Y_{W}\left(\phi_{-1}^{i} \mathbf{1}, z\right)=\phi_{W}^{i}(z)$ for $i \in I$.

Proof. The proof of this theorem is similar to the proof of Theorem 3.5 in [H2] but is more complicated because the twisted vertex operator map is multivalued. We refer the reader to [H6] for the definition of lower-bounded generalized $g$-twisted $V$-module.

The identity property follow from of the definition of $Y_{W}^{g}$.

Let $L_{W}^{g}(0)^{\prime}$ be the adjoint operator of $L_{W}^{g}(0)$. For $w^{\prime} \in W^{\prime}, w \in W, i_{1}, \ldots, i_{k} \in I$ and $n_{1}, \ldots, n_{k} \in \mathbb{Z}, q_{1}, \ldots, q_{k} \in \mathbb{N}, c \in \mathbb{C}$,

$$
\begin{aligned}
& \left\langle w^{\prime}, e^{c L_{W}^{g}(0)}\left(Y_{W}^{g}\right)^{p}\left(\phi_{n_{1}}^{i_{1}} \cdots \phi_{n_{k}}^{i_{k}} \mathbf{1}, z\right) e^{-c L_{W}^{g}(0)} w\right\rangle \\
& =\left\langle e^{c L_{W}^{g}(0)^{\prime}} w^{\prime},\left(Y_{W}^{g}\right)^{p}\left(\phi_{n_{1}}^{i_{1}} \cdots \phi_{n_{k}}^{i_{k}} \mathbf{1}, z\right) e^{-c L_{W}^{g}(0)} w\right\rangle \\
& =\operatorname{Res}_{\xi_{1}=0} \cdots \operatorname{Res}_{\xi_{k}=0} \xi_{1}^{n_{1}} \cdots \xi_{k}^{n_{k}} F^{p}\left(\left\langle e^{c L_{W}^{g}(0)^{\prime}} w^{\prime}, \phi_{W}^{i_{1}}\left(\xi_{1}+z\right) \cdots \phi_{W}^{i_{k}}\left(\xi_{k}+z\right) e^{-c L_{W}^{g}(0)} w\right\rangle\right) \\
& =\operatorname{Res}_{\xi_{1}=0} \cdots \operatorname{Res}_{\xi_{k}=0} \xi_{1}^{n_{1}} \cdots \xi_{k}^{n_{k}} F^{p}\left(\left\langle v^{\prime}, e^{c L_{W}^{g}(0)} \phi_{W}^{i_{1}}\left(\xi_{1}+z\right) \cdots \phi_{W}^{i_{k}}\left(\xi_{k}+z\right) e^{-c L_{W}^{g}(0)} w\right\rangle\right) \\
& =\operatorname{Res}_{\xi_{1}=0} \cdots \operatorname{Res}_{\xi_{k}=0} \xi_{1}^{n_{1}} \cdots \xi_{k}^{n_{k}} a^{c\left(\operatorname{wt}^{i_{1}}+\cdots \operatorname{wt} \phi^{i_{k}}\right)} F^{p}\left(\left\langle w^{\prime}, \phi_{W}^{i_{1}}\left(a \xi_{1}+a z\right) \cdots \phi_{W}^{i_{k}}\left(a \xi_{k}+a z\right) w\right\rangle\right)
\end{aligned}
$$




$$
\begin{aligned}
& =\operatorname{Res}_{\zeta_{1}=0} \cdots \operatorname{Res}_{\zeta_{k}=0} \zeta_{1}^{n_{1}} \cdots \zeta_{k}^{n_{k}} a^{\mathrm{wt} \phi^{i_{1}}+\cdots \mathrm{wt} \phi^{i_{k}-k-n_{1}-\cdots-n_{k}} .} \\
& \quad \cdot F^{p}\left(\left\langle w^{\prime}, \phi_{W}^{i_{1}}\left(\zeta_{1}+a z\right) \cdots \phi_{W}^{i_{k}}\left(\zeta_{k}+a z\right) w\right\rangle\right) \\
& \left.=\left\langle w^{\prime},\left(Y_{W}^{g}\right)^{p}\left(e^{c L_{V}(0)} \phi_{n_{1}}^{i_{1}} \cdots \phi_{n_{k}}^{i_{k}} \mathbf{1}, a z\right) w\right\rangle\right) .
\end{aligned}
$$

This formula is equivalent to the $L(0)$-commutator formula.

From Property 2 in Assumption 2.3 and the definition of $\left(Y_{W}^{g}\right)^{p}$, we obtain the $L(-1)$ commutator formula

$$
\frac{d}{d z}\left(Y_{W}^{g}\right)^{p}\left(\phi_{n_{1}}^{i_{1}} \cdots \phi_{n_{k}}^{i_{k}} \mathbf{1}, z\right)=\left[L_{W}^{g}(-1),\left(Y_{W}^{g}\right)^{p}\left(\phi_{n_{1}}^{i_{1}} \cdots \phi_{n_{k}}^{i_{k}} \mathbf{1}, z\right)\right]
$$

Let $\left\{e_{n}\right\}_{n \in \mathbb{Z}}$ be a homogeneous basis of $W$ and $\left\{e_{n}^{\prime}\right\}_{n \in \mathbb{Z}}$ its dual basis in $W^{\prime}$. Then we have

$$
\begin{aligned}
& \left\langle w^{\prime},\left(Y_{W}^{g}\right)^{p}\left(\phi_{n_{1}}^{i_{1}} \cdots \phi_{n_{k}}^{i_{k}} \mathbf{1}, z_{1}\right)\left(Y_{W}^{g}\right)^{p}\left(\phi_{m_{1}}^{j_{1}} \cdots \phi_{m_{l}}^{j_{l}} \mathbf{1}, z_{2}\right) w\right\rangle \\
& =\sum_{n \in \mathbb{Z}}\left\langle w^{\prime},\left(Y_{W}^{g}\right)^{p}\left(\phi_{n_{1}}^{i_{1}} \cdots \phi_{n_{k}}^{i_{k}} \mathbf{1}, z_{1}\right) e_{n}\right\rangle\left\langle e_{n}^{\prime},\left(Y_{W}^{g}\right)^{p}\left(\phi_{m_{1}}^{j_{1}} \cdots \phi_{m_{l}}^{j_{l}} \mathbf{1}, z_{2}\right) w\right\rangle \\
& =\sum_{n \in \mathbb{Z}} \operatorname{Res}_{\zeta_{1}=0} \cdots \operatorname{Res}_{\zeta_{k}=0} \zeta_{1}^{n_{1}} \cdots \zeta_{k}^{n_{k}} \operatorname{Res}_{\xi_{1}=0} \cdots \operatorname{Res}_{\xi_{l}=0} \xi_{1}^{m_{1}} \cdots \xi_{l}^{m_{l}} . \\
& \quad \cdot F^{p}\left(\left\langle w^{\prime}, \phi_{W}^{i_{1}}\left(\zeta_{1}+z_{1}\right) \cdots \phi_{W}^{i_{k}}\left(\zeta_{k}+z_{1}\right) e_{n}\right\rangle\right) F^{p}\left(\left\langle e_{n}^{\prime}, \phi_{W}^{j_{1}}\left(\xi_{1}+z_{2}\right) \cdots \phi_{W}^{j_{l}}\left(\xi_{l}+z_{2}\right) w\right\rangle\right) \\
& =\operatorname{Res}_{\zeta_{1}=0} \cdots \operatorname{Res}_{\zeta_{k}=0} \zeta_{1}^{n_{1}} \cdots \zeta_{k}^{n_{k}} \operatorname{Res}_{\xi_{1}=0} \cdots \operatorname{Res}_{\xi_{l}=0} \xi_{1}^{m_{1}} \cdots \xi_{l}^{m_{l}} \cdot \\
& \quad \cdot \sum_{n \in \mathbb{Z}} F^{p}\left(\left\langle w^{\prime}, \phi_{W}^{i_{1}}\left(\zeta_{1}+z_{1}\right) \cdots \phi_{W}^{i_{k}}\left(\zeta_{k}+z_{1}\right) e_{n}\right\rangle\right) F^{p}\left(\left\langle e_{n}^{\prime}, \phi_{W}^{j_{1}}\left(\xi_{1}+z_{2}\right) \cdots \phi_{W}^{j_{l}}\left(\xi_{l}+z_{2}\right) w\right\rangle\right) .
\end{aligned}
$$

By Property 13 in Theorem 3.1 , when $\left|z_{1}\right|>\cdots>\left|z_{k+l}\right|>0$,

$$
\begin{gathered}
\sum_{n \in \mathbb{Z}} F^{p}\left(\left\langle w^{\prime}, \phi_{W}^{i_{1}}\left(z_{1}\right) \cdots \phi_{W}^{i_{k}}\left(z_{k}\right) e_{n}\right\rangle\right) F^{p}\left(\left\langle e_{n}^{\prime}, \phi_{W}^{j_{1}}\left(z_{k+1}\right) \cdots \phi_{W}^{j_{l}}\left(z_{k+l}\right) w\right\rangle\right) \\
=\sum_{n \in \mathbb{Z}}\left\langle w^{\prime}, \phi_{W}^{i_{1}}\left(z_{1}\right) \cdots \phi_{W}^{i_{k}}\left(z_{k}\right) e_{n}\right\rangle\left\langle e_{n}^{\prime}, \phi_{W}^{j_{1}}\left(z_{k+1}\right) \cdots \phi_{W}^{j_{l}}\left(z_{k+l}\right) w\right\rangle \\
=\left\langle w^{\prime}, \phi_{W}^{i_{1}}\left(z_{1}\right) \cdots \phi_{W}^{i_{k}}\left(z_{k}\right) \phi_{W}^{j_{1}}\left(z_{k+1}\right) \cdots \phi_{W}^{j_{l}}\left(z_{k+l}\right) w\right\rangle
\end{gathered}
$$

is absolutely convergent to the analytic function

$$
F^{p}\left(\left\langle w^{\prime}, \phi_{W}^{i_{1}}\left(z_{1}\right) \cdots \phi_{W}^{i_{k}}\left(z_{k}\right) \phi_{W}^{j_{1}}\left(z_{k+1}\right) \cdots \phi_{W}^{j_{l}}\left(z_{k+l}\right) w\right\rangle\right)
$$

in $z_{1}, \ldots, z_{k+l}$. On the other hand, also by Property 13 in Theorem 3.1, there is a unique expansion of this branch of a multivalued function in the region $\left|z_{1}\right|, \ldots,\left|z_{k}\right|>\left|z_{k+1}\right|, \ldots,\left|z_{k+l}\right|>$ $0, z_{i} \neq z_{j}$ for $i \neq j, i, j=1, \ldots, k$ and $i, j=k+1, \ldots, k+l$ such that each term is a product of two analytic functions of the same form, one in $z_{1}, \ldots, z_{k}$ and the other in $z_{k+1}, \ldots, z_{k+l}$. Since the left-hand side of (4.16) is a series of the same form and is absolutely convergent in the region $\left|z_{1}\right|>\cdots>\left|z_{k+l}\right|>0$ to (4.17), it must be absolutely convergent in the 
larger region $\left|z_{1}\right|, \ldots,\left|z_{k}\right|>\left|z_{k+1}\right|, \ldots,\left|z_{k+l}\right|>0, z_{i} \neq z_{j}$ for $i \neq j, i, j=1, \ldots, k$ and $i, j=k+1, \ldots, k+l$ to (4.17).

Substituting $\zeta_{i}+z_{1}$ for $z_{i}$ for $i=1, \ldots, k$ and $\xi_{j}+z_{2}$ for $z_{k+j}$ for $j=1, \ldots, l$, we see that

$$
\sum_{n \in \mathbb{Z}} F^{p}\left(\left\langle w^{\prime}, \phi_{W}^{i_{1}}\left(\zeta_{1}+z_{1}\right) \cdots \phi_{W}^{i_{k}}\left(\zeta_{k}+z_{1}\right) e_{n}\right\rangle\right) F^{p}\left(\left\langle e_{n}^{\prime}, \phi_{W}^{j_{1}}\left(\xi_{1}+z_{2}\right) \cdots \phi_{W}^{j_{l}}\left(\xi_{l}+z_{2}\right) w\right\rangle\right)
$$

is absolutely convergent to

$$
F^{p}\left(\left\langle w^{\prime}, \phi_{W}^{i_{1}}\left(\zeta_{1}+z_{1}\right) \cdots \phi_{W}^{i_{k}}\left(\zeta_{k}+z_{1}\right) \phi_{W}^{j_{1}}\left(\xi_{1}+z_{2}\right) \cdots \phi_{W}^{j_{l}}\left(\xi_{l}+z_{2}\right) w\right\rangle\right)
$$

when $\left|\zeta_{1}+z_{1}\right|, \ldots,\left|\zeta_{k}+z_{1}\right|>\left|\xi_{1}+z_{2}\right|, \ldots,\left|\xi_{l}+z_{2}\right|>0, \zeta_{i} \neq \zeta_{j}$ for $i, j=1, \ldots, k$ and $\xi_{i} \neq \xi_{j}$ for $i, j=1, \ldots, l$. When $\left|z_{1}\right|>\left|z_{2}\right|>0$, we can always find sufficiently small neighborhood of 0 such that when $\zeta_{1}, \ldots, \zeta_{k}, \xi_{1}, \ldots, \xi_{l}$ are in this neighborhood, $\left|\zeta_{1}+z_{1}\right|, \ldots,\left|\zeta_{k}+z_{1}\right|>$ $\left|\xi_{1}+z_{2}\right|, \ldots,\left|\xi_{l}+z_{2}\right|>0$ holds. Thus we see that when $\left|z_{1}\right|>\left|z_{2}\right|>0$, the right-hand side of (4.15) is absolutely convergent to

$$
\begin{aligned}
\operatorname{Res}_{\zeta_{1}=0} \cdots \operatorname{Res}_{\zeta_{k}=0} \zeta_{1}^{n_{1}} \cdots \zeta_{k}^{n_{k}} \operatorname{Res}_{\xi_{1}=0} \cdots \operatorname{Res}_{\xi_{l}=0} \xi_{1}^{m_{1}} \cdots \xi_{l}^{m_{l}} & \\
\cdot & F^{p}\left(\left\langle w^{\prime}, \phi_{W}^{i_{1}}\left(\zeta_{1}+z_{1}\right) \cdots \phi_{W}^{i_{k}}\left(\zeta_{k}+z_{1}\right) \phi_{W}^{j_{1}}\left(\xi_{1}+z_{2}\right) \cdots \phi_{W}^{j_{l}}\left(\xi_{l}+z_{2}\right) w\right\rangle\right) .
\end{aligned}
$$

From the explicit expression of

$$
F^{p}\left(\left\langle w^{\prime}, \phi_{W}^{i_{1}}\left(\zeta_{1}+z_{1}\right) \cdots \phi_{W}^{i_{k}}\left(\zeta_{k}+z_{1}\right) \phi_{W}^{j_{1}}\left(\xi_{1}+z_{2}\right) \cdots \phi_{W}^{j_{l}}\left(\xi_{l}+z_{2}\right) w\right\rangle\right)
$$

(see Property 13 in Theorem 3.1), it is clear that (4.18) is an analytic function in $z_{1}$ and $z_{2}$ of the form

$$
\sum_{i, j, k, l=0}^{N} a_{i j k l} e^{m_{i} l_{p}\left(z_{1}\right)} e^{n_{j} l_{p}\left(z_{2}\right)} l_{p}\left(z_{1}\right)^{k} l_{p}\left(z_{2}\right)^{l}\left(z_{1}-z_{2}\right)^{-t} .
$$

In particular, the left-hand side of (4.15), that is,

$$
\left\langle w^{\prime},\left(Y_{W}^{g}\right)^{p}\left(\phi_{n_{1}}^{i_{1}} \cdots \phi_{n_{k}}^{i_{k}} \mathbf{1}, z_{1}\right)\left(Y_{W}^{g}\right)^{p}\left(\phi_{m_{1}}^{j_{1}} \cdots \phi_{m_{l}}^{j_{l}} \mathbf{1}, z_{2}\right) w\right\rangle,
$$

is absolutely convergent in the region $\left|z_{1}\right|>\left|z_{2}\right|>0$ to this analytic function.

We have proved that the product of two vertex operators is convergent to an analytic function of the form (4.19), or equivalently, the corresponding branch of a multivalued function with preferred branch of the form

$$
f\left(z_{1}, z_{2}\right)=\sum_{i, j, k, l=0}^{N} a_{i j k l} z_{1}^{m_{i}} z_{2}^{n_{j}}\left(\log z_{1}\right)^{k}\left(\log z_{2}\right)^{l}\left(z_{1}-z_{2}\right)^{-t} .
$$

We are ready to prove the commutativity. The calculation above also shows that

$$
\left\langle w^{\prime},\left(Y_{W}^{g}\right)^{p}\left(\phi_{m_{1}}^{j_{1}} \cdots \phi_{m_{l}}^{j_{l}} \mathbf{1}, z_{2}\right)\left(Y_{W}^{g}\right)^{p}\left(\phi_{n_{1}}^{i_{1}} \cdots \phi_{n_{k}}^{i_{k}} \mathbf{1}, z_{1}\right) w\right\rangle
$$


is absolutely convergent to the rational function

$$
\begin{aligned}
& \operatorname{Res}_{\xi_{1}=0} \cdots \operatorname{Res}_{\xi_{l}=0} \xi_{1}^{m_{1}} \cdots \xi_{l}^{m_{l}} \operatorname{Res}_{\zeta_{1}=0} \cdots \operatorname{Res}_{\zeta_{k}=0} \zeta_{1}^{n_{1}} \cdots \zeta_{k}^{n_{k}} \cdot \\
& \cdot F^{p}\left(\left\langle w^{\prime}, \phi_{W}^{j_{1}}\left(\xi_{1}+z_{2}\right) \cdots \phi_{W}^{j_{l}}\left(\xi_{l}+z_{2}\right) \phi_{W}^{i_{1}}\left(\zeta_{1}+z_{1}\right) \cdots \phi_{W}^{i_{k}}\left(\zeta_{k}+z_{1}\right) w\right\rangle\right),
\end{aligned}
$$

in the regions $\left|z_{2}\right|>\left|z_{1}\right|>0$, respectively. By Property 14 in Theorem 3.1, the analytic functions (4.18) and (4.22) multiplied by

$$
\prod_{r=1}^{k} \prod_{s=1}^{l}(-1)^{\left|\phi^{i_{r}}\right|\left|\phi^{j_{s}}\right|}=(-1)^{\left|\phi_{n_{1}}^{i_{1}} \cdots \phi_{n_{k}}^{i_{k}} \mathbf{1}\right|\left|\phi_{m_{1}}^{j_{1}} \cdots \phi_{m_{l}}^{j_{l}} \mathbf{1}\right|}
$$

are equal. Thus (4.20) and (4.21) multiplied by the sign $(-1)^{\left|\phi_{n_{1}}^{i_{1}} \cdots \phi_{n_{k}}^{i_{k}} \mathbf{1} 1\right| \phi_{m_{1}}^{j_{1}} \cdots \phi_{m_{l}}^{j_{l}} \mathbf{1} \mid}$ are absolutely convergent in the regions $\left|z_{1}\right|>\left|z_{2}\right|>0$ and $\left|z_{2}\right|>\left|z_{1}\right|>0$, respectively, to a common analytic function of the form (4.19).

We now prove the associativity. For $i_{1}, \ldots, i_{k}, j_{1}, \ldots, j_{l} \in I, m_{1}, \ldots, m_{l} \in \mathbb{Z}, v \in V$ and $v^{\prime} \in V^{\prime}$, using the expansion of $\phi^{i_{1}}\left(\xi_{1}\right), \ldots, \phi^{i_{k}}\left(\xi_{k}\right)$ and the definition of $\left(Y_{W}^{g}\right)^{p}$, we have

$$
\begin{aligned}
& \left\langle w^{\prime},\left(Y_{W}^{g}\right)^{p}\left(\phi^{i_{1}}\left(z_{1}\right) \cdots \phi^{i_{k}}\left(z_{k}\right) \phi_{m_{1}}^{j_{1}} \cdots \phi_{m_{l}}^{j_{l}} \mathbf{1}, z\right) w\right\rangle \\
& =\sum_{p_{1}, \ldots, p_{k} \in \mathbb{Z}}\left\langle w^{\prime},\left(Y_{W}^{g}\right)^{p}\left(\phi_{p_{1}}^{i_{1}} \cdots \phi_{p_{k}}^{i_{k}} \phi_{m_{1}}^{j_{1}} \cdots \phi_{m_{l}}^{j_{l}} \mathbf{1}, z\right) w\right\rangle z_{1}^{-p_{1}-1} \cdots z_{k}^{-p_{k}-1} \\
& =\sum_{p_{1}, \ldots, p_{k} \in \mathbb{Z}} \operatorname{Res}_{\zeta_{1}=0} \cdots \operatorname{Res}_{\zeta_{k}=0} \zeta_{1}^{p_{1}} \cdots \zeta_{k}^{p_{k}} \operatorname{Res}_{\xi_{1}=0} \cdots \operatorname{Res}_{\xi_{l}=0} \xi_{1}^{m_{1}} \cdots \xi_{l}^{m_{l}} . \\
& \quad \cdot F^{p}\left(\left\langle w^{\prime}, \phi_{W}^{i_{1}}\left(\zeta_{1}+z\right) \cdots \phi_{W}^{i_{k}}\left(\zeta_{k}+z\right) \phi_{W}^{j_{1}}\left(\xi_{1}+z\right) \cdots \phi_{W}^{j_{l}}\left(\xi_{l}+z\right) w\right\rangle\right) z_{1}^{-p_{1}-1} \cdots z_{k}^{-p_{k}-1} .
\end{aligned}
$$

We now expand

$$
F^{p}\left(\left\langle w^{\prime}, \phi_{W}^{i_{1}}\left(\zeta_{1}+z\right) \cdots \phi_{W}^{i_{k}}\left(\zeta_{k}+z\right) \phi_{W}^{j_{1}}\left(\xi_{1}+z\right) \cdots \phi_{W}^{j_{l}}\left(\xi_{l}+z\right) w\right\rangle\right)
$$

as a Laurent series

$$
\sum_{l \in \mathbb{Z}} f_{l}\left(\zeta_{1}, \ldots, \zeta_{k-1}, \xi_{1}, \ldots, \xi_{l}, z\right) \zeta_{k}^{-l-1}
$$

in $\zeta_{k}$ in the region $|z|,\left|\zeta_{1}\right|, \ldots,\left|\zeta_{k-1}\right|>\left|\zeta_{k}\right|>\left|\xi_{1}\right|, \ldots,\left|\xi_{l}\right|$, where $f_{l}\left(\zeta_{1}, \ldots, \zeta_{k-1}, \xi_{1}, \ldots, \xi_{l}, z\right)$ are analytic functions in $\zeta_{1}, \ldots, \zeta_{k-1}, \xi_{1}, \ldots, \xi_{l}$ and $z$. Then in the region that the Laurent series expansion holds, we have

$$
\begin{aligned}
& \sum_{p_{k} \in \mathbb{Z}} \operatorname{Res}_{\zeta_{k}=0} \zeta_{k}^{p_{k}}\left(\sum_{l \in \mathbb{Z}} f_{l}\left(\zeta_{1}, \ldots, \zeta_{k-1}, \xi_{1}, \ldots, \xi_{l}, z\right) \zeta_{k}^{-l-1}\right) z_{k}^{-p_{k}-1} \\
& \quad=\sum_{p_{k} \in \mathbb{Z}} f_{p_{k}}\left(\zeta_{1}, \ldots, \zeta_{k-1}, \xi_{1}, \ldots, \xi_{l}, z\right) z_{k}^{-p_{k}-1} \\
& \quad=F^{p}\left(\left\langle w^{\prime}, \phi_{W}^{i_{1}}\left(\zeta_{1}+z\right) \cdots \phi_{W}^{i_{k-1}}\left(\zeta_{k-1}+z\right) \phi_{W}^{i_{k}}\left(z_{k}+z\right) \phi_{W}^{j_{1}}\left(\xi_{1}+z\right) \cdots \phi_{W}^{j_{l}}\left(\xi_{l}+z\right) w\right\rangle\right) .
\end{aligned}
$$


Repeating this step for the variables $\zeta_{k-1}, \ldots, \zeta_{1}$, we see that the right-hand side of (4.23) is equal to the expansion of

$$
\operatorname{Res}_{\xi_{1}=0} \cdots \operatorname{Res}_{\xi_{l}=0} \xi_{1}^{m_{1}} \cdots \xi_{l}^{m_{l}} F^{p}\left(\left\langle w^{\prime}, \phi_{W}^{i_{1}}\left(z_{1}+z\right) \cdots \phi_{W}^{i_{k}}\left(z_{k}+z\right) \phi_{W}^{j_{1}}\left(\xi_{1}+z\right) \cdots \phi_{W}^{j_{l}}\left(\xi_{l}+z\right) w\right\rangle\right)
$$

as a Laurent series in $z_{1} \ldots, z_{k}$ in the region $|z|>\left|z_{1}\right|>\cdots>\left|z_{k}\right|>0$. Thus the lefthand side of (4.23) is absolutely convergent to (4.25) in the region for this Laurent series expansion, that is, in the region $|z|>\left|z_{1}\right|>\cdots>\left|z_{k}\right|>0$,

$$
\begin{aligned}
& \left\langle w^{\prime},\left(Y_{W}^{g}\right)^{p}\left(\phi^{i_{1}}\left(z_{1}\right) \cdots \phi^{i_{k}}\left(z_{k}\right) \phi_{m_{1}}^{j_{1}} \cdots \phi_{m_{l}}^{j_{l}} \mathbf{1}, z\right) w\right\rangle \\
& =\operatorname{Res}_{\xi_{1}=0} \cdots \operatorname{Res}_{\xi_{l}=0} \xi_{1}^{m_{1}} \cdots \xi_{l}^{m_{l}} \cdot \\
& \quad \cdot F^{p}\left(\left\langle w^{\prime}, \phi_{W}^{i_{1}}\left(z_{1}+z\right) \cdots \phi_{W}^{i_{k}}\left(z_{k}+z\right) \phi_{W}^{j_{1}}\left(\xi_{1}+z\right) \cdots \phi_{W}^{j_{l}}\left(\xi_{l}+z\right) w\right\rangle\right) .
\end{aligned}
$$

On the other hand, we have

$$
\begin{aligned}
& \left\langle w^{\prime},\left(Y_{W}^{g}\right)^{p}\left(Y_{V}\left(\phi_{n_{1}}^{i_{1}} \cdots \phi_{n_{k}}^{i_{k}} \mathbf{1}, z_{1}-z_{2}\right) \phi_{m_{1}}^{j_{1}} \cdots \phi_{m_{l}}^{j_{l}} \mathbf{1}, z_{2}\right) w\right\rangle \\
& =\sum_{n \in \mathbb{Z}}\left\langle w^{\prime},\left(Y_{W}^{g}\right)^{p}\left(e_{n}, z_{2}\right) w\right\rangle\left\langle e_{n}^{\prime}, Y_{V}\left(\phi_{n_{1}}^{i_{1}} \cdots \phi_{n_{k}}^{i_{k}} \mathbf{1}, z_{1}-z_{2}\right) \phi_{m_{1}}^{j_{1}} \cdots \phi_{m_{l}}^{j_{l}} \mathbf{1}\right\rangle \\
& =\sum_{n \in \mathbb{Z}}\left\langle w^{\prime},\left(Y_{W}^{g}\right)^{p}\left(e_{n}, z_{2}\right) w\right\rangle \operatorname{Res}_{\zeta_{1}=0} \cdots \operatorname{Res}_{\zeta_{k}=0} \zeta_{1}^{n_{1}} \cdots \zeta_{k}^{n_{k}} \cdot \\
& \cdot R\left(\left\langle e_{n}^{\prime}, \phi^{i_{1}}\left(\zeta_{1}+z_{1}-z_{2}\right) \cdots \phi^{i_{k}}\left(\zeta_{k}+z_{1}-z_{2}\right) \phi_{m_{1}}^{j_{1}} \cdots \phi_{m_{l}}^{j_{l}} \mathbf{1}\right\rangle\right),
\end{aligned}
$$

where we have used the definition of $Y_{V}$ in [H2]. But by (4.26), in the region $\left|z_{2}\right|>\mid \zeta_{1}+$ $z_{1}-z_{2}|>\cdots>| \zeta_{k}+z_{1}-z_{2}|>0,| \arg \left(\zeta_{k}+z_{1}\right)-\arg z_{2}\left|<\frac{\pi}{2}, \ldots,\right| \arg \left(\zeta_{1}+z_{1}\right)-\arg z_{2} \mid<\frac{\pi}{2}$, we have

$$
\begin{aligned}
\sum_{n \in \mathbb{Z}}\left\langle w^{\prime},\left(Y_{W}^{g}\right)^{p}\left(e_{n}, z_{2}\right) w\right\rangle\left\langle e_{n}^{\prime}, \phi^{i_{1}}\left(\zeta_{1}+z_{1}-z_{2}\right) \cdots \phi^{i_{k}}\left(\zeta_{k}+z_{1}-z_{2}\right) \phi_{m_{1}}^{j_{1}} \cdots \phi_{m_{l}}^{j_{l}} \mathbf{1}\right\rangle \\
=\left\langle w^{\prime},\left(Y_{W}^{g}\right)^{p}\left(\phi^{i_{1}}\left(\zeta_{1}+z_{1}-z_{2}\right) \cdots \phi^{i_{k}}\left(\zeta_{k}+z_{1}-z_{2}\right) \phi_{m_{1}}^{j_{1}} \cdots \phi_{m_{l}}^{j_{l}} \mathbf{1}, z_{2}\right) w\right\rangle \\
=\operatorname{Res}_{\xi_{1}=0} \cdots \operatorname{Res}_{\xi_{l}=0} \xi_{1}^{m_{1}} \cdots \xi_{l}^{m_{l}} \cdot \\
\quad \cdot F^{p}\left(\left\langle w^{\prime}, \phi_{W}^{i_{1}}\left(\zeta_{1}+z_{1}\right) \cdots \phi_{W}^{i_{k}}\left(\zeta_{k}+z_{1}\right) \phi_{W}^{j_{1}}\left(\xi_{1}+z_{2}\right) \cdots \phi_{W}^{j_{l}}\left(\xi_{l}+z_{2}\right) w\right\rangle\right) .
\end{aligned}
$$

The right-hand side of (4.28) is an analytic function in $\zeta_{1}, \ldots, \zeta_{k}, z_{1}$ and $z_{2}$ of the form

$$
\begin{gathered}
\sum_{i_{1}, \ldots, i_{k}, i, n_{1}, \ldots, n_{k}, n=0}^{N} f_{i_{1} \cdots i_{k} i n_{1} \cdots n_{k} n}\left(\zeta_{1}+z_{1}, \ldots, \zeta_{k}+z_{1}, z_{2}\right) \cdot \\
\cdot e^{r_{i_{1}}^{(1)} l_{p}\left(\zeta_{1}+z_{1}\right)} \cdots e^{r_{i_{k}}^{(k)} l_{p}\left(\zeta_{k}+z_{1}\right)} e^{r_{i} l_{p}\left(z_{2}\right)}\left(l_{p}\left(\zeta_{1}+z_{1}\right)\right)^{n_{1}} \cdots\left(l_{p}\left(\zeta_{k}+z_{1}\right)\right)^{n_{k}}\left(l_{p}\left(z_{2}\right)\right)^{n}
\end{gathered}
$$

where $f_{i_{1} \cdots i_{k} i n_{1} \cdots n_{k} n}\left(\zeta_{1}+z_{1}, \ldots, \zeta_{k}+z_{1}, z_{1}, z_{2}\right)$ for $i_{1}, \ldots, i_{k}, i, n_{1}, \ldots, n_{k}, n=0, \ldots, N$ are rational functions in $\zeta_{1}, \ldots, \zeta_{k}, z_{1}, z_{2}$ with the only possible poles $\zeta_{i}-\zeta_{j}=0$ for $i \neq j$ and $\zeta_{i}+z_{1}-z_{2}=0$. There is a unique expansion of such an analytic function in the region $\left|z_{2}\right|>$ 
$\left|\zeta_{1}+z_{1}-z_{2}\right|, \ldots,\left|\zeta_{k}+z_{1}-z_{2}\right|>0,\left|\arg \left(\zeta_{k}+z_{1}\right)-\arg z_{2}\right|<\frac{\pi}{2}, \ldots,\left|\arg \left(\zeta_{1}+z_{1}\right)-\arg z_{2}\right|<\frac{\pi}{2}$, $\zeta_{i} \neq \zeta_{j}$ for $i \neq j, i, j=1, \ldots, k$, such that each term is a product of two analytic functions, one being analytic in $z_{2}$ of the form

$$
\sum_{j, q=0}^{M} b_{j q} e^{s_{j} l_{p}\left(z_{2}\right)}\left(l_{p}\left(z_{2}\right)\right)^{q}
$$

and the other being a rational function in $\zeta_{1}+z_{1}-z_{2}, \ldots, \zeta_{k}+z_{1}-z_{2}$ and $z_{1}$ with the only possible poles $\zeta_{i}-\zeta_{j}=0$ for $i \neq j$ and $\zeta_{i}+z_{1}-z_{2}=0$. Since

$$
\sum_{n \in \mathbb{Z}}\left\langle w^{\prime},\left(Y_{W}^{g}\right)^{p}\left(e_{n}, z_{2}\right) w\right\rangle R\left(\left\langle e_{n}^{\prime}, \phi^{i_{1}}\left(\zeta_{1}+z_{1}-z_{2}\right) \cdots \phi^{i_{k}}\left(\zeta_{k}+z_{1}-z_{2}\right) \phi_{m_{1}}^{j_{1}} \cdots \phi_{m_{l}}^{j_{l}} \mathbf{1}\right\rangle\right)
$$

is a series of the same form and is equal to the left-hand side of (4.28) in the region $\left|z_{2}\right|>$ $\left|\zeta_{1}+z_{1}-z_{2}\right|>\cdots>\left|\zeta_{k}+z_{1}-z_{2}\right|>0$, it must be absolutely convergent to the right-hand side of (4.28) in the larger region $\left|z_{2}\right|>\left|\zeta_{1}+z_{1}-z_{2}\right|, \ldots,\left|\zeta_{k}+z_{1}-z_{2}\right|>0,\left|\arg \left(\zeta_{k}+z_{1}\right)-\arg z_{2}\right|<$ $\frac{\pi}{2}, \ldots,\left|\arg \left(\zeta_{1}+z_{1}\right)-\arg z_{2}\right|<\frac{\pi}{2}$. Therefore we obtain

$$
\begin{aligned}
\sum_{n \in \mathbb{Z}}\left\langle w^{\prime},\left(Y_{W}^{g}\right)^{p}\left(e_{n}, z_{2}\right) w\right\rangle R\left(\left\langle e_{n}^{\prime}, \phi^{i_{1}}\left(\zeta_{1}+z_{1}-z_{2}\right) \cdots \phi^{i_{k}}\left(\zeta_{k}+z_{1}-z_{2}\right) \phi_{m_{1}}^{j_{1}} \cdots \phi_{m_{l}}^{j_{l}} \mathbf{1}\right\rangle\right) \\
=\operatorname{Res}_{\xi_{1}=0} \cdots \operatorname{Res}_{\xi_{l}=0} \xi_{1}^{m_{1}} \cdots \xi_{l}^{m_{l}} \cdot \\
\cdot F^{p}\left(\left\langle w^{\prime}, \phi_{W}^{i_{1}}\left(\zeta_{1}+z_{1}\right) \cdots \phi_{W}^{i_{k}}\left(\zeta_{k}+z_{1}\right) \phi_{W}^{j_{1}}\left(\xi_{1}+z_{2}\right) \cdots \phi_{W}^{j_{l}}\left(\xi_{l}+z_{2}\right) w\right\rangle\right) .
\end{aligned}
$$

in the region $\left|z_{2}\right|>\left|\zeta_{1}+z_{1}-z_{2}\right|, \ldots,\left|\zeta_{k}+z_{1}-z_{2}\right|>0$. Thus when $\left|z_{2}\right|>\left|z_{1}-z_{2}\right|>0$, the right-hand side of (4.27) is absolutely convergent to

$$
\begin{aligned}
& \operatorname{Res}_{\zeta_{1}=0} \cdots \operatorname{Res}_{\zeta_{k}=0} \zeta_{1}^{n_{1}} \cdots \zeta_{k}^{n_{k}} \operatorname{Res}_{\xi_{1}=0} \cdots \operatorname{Res}_{\xi_{l}=0} \xi_{1}^{m_{1}} \cdots \xi_{l}^{m_{l}} \cdot \\
& \cdot F^{p}\left(\left\langle w^{\prime}, \phi_{W}^{i_{1}}\left(\zeta_{1}+z_{1}\right) \cdots \phi_{W}^{i_{k}}\left(\zeta_{k}+z_{1}\right) \phi_{W}^{j_{1}}\left(\xi_{1}+z_{2}\right) \cdots \phi_{W}^{j_{l}}\left(\xi_{l}+z_{2}\right) w\right\rangle\right),
\end{aligned}
$$

which has been proved above to be equal to the left hand side of (4.15) in the region $\left|z_{1}\right|>$ $\left|z_{2}\right|>0$. The associativity is proved.

To prove the uniqueness, we need only show that any twisted $V$-module structure on $W$ must have the vertex operator map defined by (4.7). But this is clear from the motivation that we have discussed before the definition (4.7) of the vertex operator map $Y_{W}^{g}$.

We shall say that the twisted vertex operator map $Y_{W}^{g}$ is generated by the twisted fields $\phi^{i}(x)$ for $i \in I$. The $g$-twisted $V$-module $\left(W, Y_{W}^{g}\right)$ is in fact generated by the coefficients of $\psi_{W}^{a}(x) v$ for $a \in A$ and $v \in V$.

Remark 4.4 In this paper, we formulate and prove all our results for lower-bounded generalized twisted modules mainly because the explicit construction in the next section gives in general only such twisted modules. But Theorem 4.3 can be used to construct all different classes of twisted modules. If homogeneous subspaces of $W$ are finite dimensional, we obtain a grading-restricted generalized $g$-twisted $V$-module. If in addition $L_{W}(0)$ acts on $W$ semisimply, we obtain a $g$-twisted $V$-module. In the special case that $g=1_{V}$, Theorem 4.3 can be used to construct lower-bounded generalized $V$-modules, grading-restricted generalized $V$-modules and $V$-modules. 


\section{An explicit construction of lower-bounded general- ized twisted modules satisfying a universal property}

In this section, we give an explicit construction of lower-bounded generalized $g$-twisted $V$ modules satisfying a universal property. As a consequence, every lower-bounded generalized $g$-twisted $V$-module is the quotient of such a universal lower-bounded generalized $g$-twisted $V$-module.

We still assume in this section that $V$ and $g$ satisfy Assumption 2.1. But we do not assume that we have the space, fields and operators in Data 2.2. In particular, we do not assume that Assumption 2.3 holds.

Let

$$
\hat{V}_{\phi}^{[g]}=\coprod_{i \in I, k \in \mathbb{N}} \mathbb{C} \mathcal{N}_{g}^{k} \phi_{-1}^{i} \mathbf{1} \otimes t^{\alpha^{i}} \mathbb{C}\left[t, t^{-1}\right] \oplus \mathbb{C} L_{0} \oplus \mathbb{C} L_{-1},
$$

where $L_{0}$ and $L_{-1}$ are fixed abstract basis elements of a vector space $\mathbb{C} L_{0} \oplus \mathbb{C} L_{-1}$. Let $T\left(\hat{V}_{\phi}^{[g]}\right)$ be the tensor algebra of $\hat{V}_{\phi}^{[g]}$ and let

$$
\phi_{\hat{V}_{\phi}^{[g]}}^{i}(x)=\sum_{n \in \alpha^{i}+\mathbb{Z}}\left(\left(x^{-\mathcal{N}_{g}} \phi_{-1}^{i} \mathbf{1}\right) \otimes t^{n}\right) x^{-n-1} \in x^{-\alpha^{i}} \hat{V}_{\phi}^{[g]}\left[\left[x, x^{-1}\right]\right][\log x]
$$

for $i \in I$. Then $\phi_{\hat{V}_{\phi}^{[g]}}^{i}(x)$ for $i \in I$ can be viewed as formal series of operators on $T\left(\hat{V}_{\phi}^{[g]}\right)$. Also $L_{0}$ and $L_{-1}$ can be viewed as operators on $T\left(\hat{V}_{\phi}^{[g]}\right)$. We shall use $L_{\hat{V}_{\phi}^{[g]}}(0)$ and $L_{\hat{V}_{\phi}^{[g]}}(-1)$ to denote the operators corresponding to $L_{0}$ and $L_{-1}$, respectively.

For $i, j \in I$, we can always find $M_{i, j} \in \mathbb{Z}_{+}$such that $x_{0}^{M_{i, j}} Y_{V}\left(\phi_{-1}^{i} \mathbf{1}, x_{0}\right) \phi_{-1}^{j} \mathbf{1}$ is a power series in $x_{0}$. For each pair $i, j \in I$, we choose $M_{i, j}$ to be the smallest of such positive integers. Let $J\left(\hat{V}_{\phi}^{[g]}\right)$ be the ideal of $T\left(\hat{V}_{\phi}^{[g]}\right)$ generated by the coefficients of the formal series

$$
\begin{gathered}
\left(x_{1}-x_{2}\right)^{M_{i j}} \phi_{\hat{V}_{\phi}^{[g]}}^{i}\left(x_{1}\right) \phi_{\hat{V}_{\phi}^{[g]}}^{j}\left(x_{2}\right)-(-1)^{\left|\phi^{i} \| \phi^{j}\right|}\left(x_{1}-x_{2}\right)^{M_{i j}} \phi_{\hat{V}_{\phi}^{[g]}}^{j}\left(x_{2}\right) \phi_{\hat{V}_{\phi}^{[g]}}^{i}\left(x_{1}\right), \\
L_{0} \phi_{\hat{V}_{\phi}^{[g]}}^{i}(x)-\phi_{\hat{V}_{\phi}^{[g]}}^{i}(x) L_{0}-x \frac{d}{d x} \phi_{\hat{V}_{\phi}^{[g]}}^{i}(x)-\left(\operatorname{wt} \phi^{i}\right) \phi_{\hat{V}_{\phi}^{[g]}}^{i}(x), \\
L_{-1} \phi_{\hat{V}_{\phi}^{[g]}}^{i}(x)-\phi_{\hat{V}_{\phi}^{[g]}}^{i}(x) L_{-1}-\frac{d}{d x} \phi_{\hat{V}_{\phi}^{[g]}}^{i}(x)
\end{gathered}
$$

for $i, j \in I$, where the tensor product symbol $\otimes$ is omitted. Let $U\left(\hat{V}_{\phi}^{[g]}\right)=T\left(\hat{V}_{\phi}^{[g]}\right) / J\left(\hat{V}_{\phi}^{[g]}\right)$. Then $\phi_{\hat{V}_{\phi}^{[g]}}^{i}(x), L_{\hat{V}_{\phi}^{[g]}}(0)$ and $L_{\hat{V}_{\phi}^{[g]}}(-1)$ can be viewed as formal series of operators and operators on $U\left(\hat{V}_{\phi}^{[g]}\right)$ satisfying the weak commutativity

$$
\left(x_{1}-x_{2}\right)^{M_{i j}} \phi_{\hat{V}_{\phi}^{[g]}}^{i}\left(x_{1}\right) \phi_{\hat{V}_{\phi}^{[g]}}^{j}\left(x_{2}\right)=(-1)^{\left|\phi^{i}\right|\left|\phi^{j}\right|}\left(x_{1}-x_{2}\right)^{M_{i j}} \phi_{\hat{V}_{\phi}^{[g]}}^{j}\left(x_{2}\right) \phi_{\hat{V}_{\phi}^{[g]}}^{i}\left(x_{1}\right),
$$

the $L(0)$-commutator formula

$$
L_{\hat{V}_{\phi}^{[g]}}(0) \phi_{\hat{V}_{\phi}^{[g]}}^{i}(x)-\phi_{\hat{V}_{\phi}^{[g]}}^{i}(x) L_{\hat{V}_{\phi}^{[g]}}(0)=x \frac{d}{d x} \phi_{\hat{V}_{\phi}^{[g]}}^{i}(x)+\left(\mathrm{wt} \phi^{i}\right) \phi_{\hat{V}_{\phi}^{[g]}}^{i}(x)
$$


and the $L(-1)$-commutator formula

$$
L_{\hat{V}_{\phi}^{[g]}}(-1) \phi_{\hat{V}_{\phi}^{[g]}}^{i}(x)-\phi_{\hat{V}_{\phi}^{[g]}}^{i}(x) L_{\hat{V}_{\phi}^{[g]}}(-1)=\frac{d}{d x} \phi_{\hat{V}_{\phi}^{[g]}}^{i}(x) .
$$

Let $M$ be a $\mathbb{Z}_{2}$-graded vector space (graded by $\mathbb{Z}_{2}$-fermion numbers). Assume that $g$ acts on $M$ and there is an operator $L_{M}(0)$ on $M$. If $M$ is finite dimensional, then there exist operators $\mathcal{L}_{g}, \mathcal{S}_{g}, \mathcal{N}_{g}$ such that on $M, g=e^{2 \pi i \mathcal{L}_{g}}$ and $\mathcal{S}_{g}$ and $\mathcal{N}_{g}$ are the semisimple and nilpotent, respectively, parts of $\mathcal{L}_{g}$. In this case, $M$ is also a direct sum of generalized eigenspaces for the operator $L_{M}(0)$ and $L_{M}(0)$ can be decomposed as the sum of its semisimple part $L_{M}(0)_{S}$ and nilpotent part $L_{M}(0)_{N}$. Moreover, the real parts of the eigenvalues of $L_{M}(0)$ has a lower bound. In the case that $M$ is infinite dimensional, we assume that all of these properties for $g$ and $L_{M}(0)$ hold. We call the eigenvalue of a generalized eigenvector $w \in M$ for $L_{M}(0)$ the weight of $w$ and denote it by wt $w$. Let $\left\{w^{a}\right\}_{a \in A}$ be a basis of $M$ consisting of vectors homogeneous in weights, $\mathbb{Z}_{2}$-fermion numbers and $g$-weights (eigenvalues of $g$ ) such that for $a \in A$, either $L_{M}(0)_{N} w^{a}=0$ or there exists $L_{M}(0)_{N}(a) \in A$ such that $L_{M}(0)_{N} w^{a}=w^{L_{M}(0)_{N}(a)}$. For simplicity, when $L_{M}(0)_{N} w^{a}=0$, we shall use $w^{L_{M}(0)_{N}(a)}$ to denote 0 . Then for $a \in A$, we always have $L_{M}(0)_{N} w^{a}=w^{L_{M}(0)_{N}(a)}$. For $a \in A$, let $\alpha^{a} \in \mathbb{C}$ such that $\Re\left(\alpha^{a}\right) \in[0,1)$ and $e^{2 \pi i \alpha^{a}}$ is the eigenvalue of $g$ for the generalized eigenvector $w^{a}$.

Let

$$
\widetilde{M}^{[g]}=\coprod_{\alpha \in P_{V}} U\left(\hat{V}_{\phi}^{[g]}\right) \otimes\left(M \otimes t^{\alpha} \mathbb{C}\left[t, t^{-1}\right]\right) \otimes V^{[\alpha]} .
$$

Then $\widetilde{M}^{[g]}$ is a left $U\left(\hat{V}_{\phi}^{[g]}\right)$-module. In particular, $\phi_{\hat{V}_{\phi}^{[g]}}^{i}(x), L_{\hat{V}_{\phi}^{[g]}}(0)$ and $L_{\hat{V}_{\phi}^{[g]}}(-1)$ act on $\widetilde{M}^{[g]}$ such that (5.1), (5.2) and (5.3) hold for these operators. We shall denote their actions on $\widetilde{M}^{[g]}$ by $\phi_{\widetilde{M}^{[g]}}^{i}(x), L_{\widetilde{M}^{[g]}}(0)$ and $L_{\widetilde{M}^{[g]}}(-1)$. The actions of $g, e^{2 \pi i \mathcal{S}_{g}}$ and $\mathcal{N}_{g}$ on $V$ and $M$ induce actions of $g, e^{2 \pi i \mathcal{S}_{g}}$ and $\mathcal{N}_{g}$ on $\widetilde{M}^{[g]}$.

For $i \in I$, let $K^{i} \in \mathbb{N}$ such that $\mathcal{N}_{g}^{K^{i}+1} \phi_{-1}^{i} \mathbf{1}=0$ and we denote the actions of the elements $\frac{(-1)^{k}}{k !}\left(\mathcal{N}_{g}^{k} \phi_{-1}^{i} \mathbf{1}\right) \otimes t^{n}$ for $n \in \alpha+\mathbb{Z}$ and $k=0, \ldots, K^{i}$ of $U\left(\hat{V}_{\phi}^{[g]}\right)$ on $\widetilde{M}_{\ell}^{[g]}$ by $\left(\phi_{\widetilde{M}^{[g]}}^{i}\right)_{n, k}$. For $a \in A, \alpha \in P_{V}$ and $n \in \alpha+\mathbb{Z}, w^{a} \otimes t^{n}$ can be viewed as a linear map from $V^{[\alpha]}$ to $\widetilde{M}^{[g]}$. We extend this map to a map from $V$ to $\widetilde{M}^{[g]}$ by mapping $V^{\left[\alpha^{\prime}\right]}$ to 0 for $\alpha^{\prime} \neq \alpha$. We shall denote this map by $\left(\psi_{\widetilde{M}^{[g]}}^{a}\right)_{n, 0}$. In general, for $n \in \alpha+\mathbb{Z}$ and $k \in \mathbb{N}$, we denote the linear map $v \mapsto \frac{(-1)^{k}}{k !}\left(\psi_{\widetilde{M}^{[g]}}^{a}\right)_{n, 0} \mathcal{N}_{g}^{k} v$ from $V^{[\alpha]}$ to $\widetilde{M}^{[g]}$ by $\left(\psi_{\widetilde{M}^{[g]}}^{a}\right)_{n, k}$ and extend it to a linear map from $V$ to $\widetilde{M}^{[g]}$ in the same way. Then $\widetilde{M}^{[g]}$ is spanned by elements of the form

$$
\left.\left(\phi_{\widetilde{M}^{[g]}}^{i_{1}}\right)_{n_{1}, k_{1}} \cdots\left(\phi_{\widetilde{M}^{[g]}}^{i_{l}}\right)_{n_{l}, k_{l}}\left(L_{\widetilde{M}^{[g]}}(m)\right)^{q}\right)\left(\psi_{\widetilde{M}^{[g]}}^{a}\right)_{n, k} v
$$

for $i_{1}, \ldots, i_{l} \in I, n_{1} \in \alpha^{i_{1}}+\mathbb{Z}, \ldots, n_{l} \in \alpha^{i_{l}}+\mathbb{Z}, 0 \leq k_{1} \leq K^{i_{1}}, \ldots, 0 \leq k_{l} \leq K^{i_{l}}, m=0,-1$, $q \in \mathbb{N}, a \in A, n \in \alpha+\mathbb{Z}, 0 \leq k \leq K, v \in V^{[\alpha]}, \alpha \in P_{V}$, where $K \in \mathbb{N}$ satisfying $\mathcal{N}_{g}^{K+1} v=0$. We already know that $M$ is graded by eigenvalues of $L_{M}(0)$. For the element (5.4) with homogeneous $v$, we define its weight to be

$$
\operatorname{wt} \phi^{i_{1}}-n_{1}-1+\cdots+\phi^{i_{l}}-n_{l}-1-m+\operatorname{wt} w^{a}-n-1+\operatorname{wt} v .
$$


Then

$$
\widetilde{M}^{[g]}=\coprod_{n \in \mathbb{C}} \widetilde{M}_{[n]}^{[g]},
$$

where $\widetilde{M}_{[n]}^{[g]}$ is the subspace of $\widetilde{M}^{[g]}$ consisting of elements of weight $n$.

For $i \in I$, we have the formal series of operators on $\widetilde{M}^{[g]}$

$$
\begin{aligned}
\phi_{\widetilde{M}[g]}^{i}(x) & =\sum_{k=0}^{K_{i}} \sum_{n \in \alpha^{i}+\mathbb{Z}}\left(\phi_{\widetilde{M}[g]}^{i}\right)_{n, k} x^{-n-1}(\log x)^{k} \\
& =\sum_{n \in \alpha^{i}+\mathbb{Z}}\left(\left(x^{-\mathcal{N}_{g}} \phi_{-1}^{i} \mathbf{1}\right) \otimes t^{n}\right) x^{-n-1} \\
& =\left(x^{-\mathcal{N}_{g}} \phi_{-1}^{i} \mathbf{1}\right) \otimes\left(\frac{t}{x}\right)^{\alpha^{i}} x^{-1} \delta\left(\frac{t}{x}\right) .
\end{aligned}
$$

Recall that $\phi_{\widetilde{M}^{[g]}}^{i}(x)$ is in fact the action of $\phi_{\hat{V}_{\phi}^{[g]}}^{i}(x)$ on $\widetilde{M}^{[g]}$. For $v \in V$, there exists $K^{v} \in \mathbb{N}$ such that $\mathcal{N}_{g}^{K^{v}+1} v=0$. For $a \in A$ and $v \in V^{[\alpha]}$, let

$$
\begin{aligned}
\psi_{\widetilde{M}^{[g]}}^{a}(x) v & =\sum_{k=0}^{K^{v}} \sum_{n \in \alpha+\mathbb{Z}}\left(\psi_{\widetilde{M}^{[g]}}^{a}\right)_{n, k} v x^{-n-1}(\log x)^{k} \\
& =\sum_{n \in \alpha+\mathbb{Z}}\left(w^{a} \otimes t^{n}\right) x^{-\mathcal{N}_{g}} v x^{-n-1} \\
& =\left(w^{a} \otimes\left(\frac{t}{x}\right)^{\alpha} x^{-1} \delta\left(\frac{t}{x}\right)\right)\left(x^{-\mathcal{N}_{g}} v\right) .
\end{aligned}
$$

It is a series in $x$ with coefficients in $\widetilde{M}^{[g]}$. Then $\psi_{\widetilde{M}^{[g]}}^{a}(x)$ is a formal series with coefficients in $\operatorname{Hom}\left(V, \widetilde{M}^{[g]}\right)$.

Let $B \in \mathbb{R}$ such that $B \leq \Re($ wt $w)$ for any generalized eigenvector $w \in M$ of $L_{M}(0)$. Such $B$ exists because the real parts of the eigenvalues of $L_{M}(0)$ is lower bounded. Let $J_{B}\left(\widetilde{M}^{[g]}\right)$ be the $U\left(\hat{V}_{\phi}^{[g]}\right)$-submodule of $\widetilde{M}^{[g]}$ generated by elements of the following forms: (i) $\left(\psi_{\widetilde{M}^{[g]}}^{a}\right)_{n, 0} \mathbf{1}$ for $a \in A$, and $n \notin-\mathbb{N}-1$; (ii) (5.4) for $i_{1}, \ldots, i_{l} \in I, n_{1} \in \alpha^{i_{1}}+\mathbb{Z}, \ldots, n_{l} \in \alpha^{i_{l}}+\mathbb{Z}$, $0 \leq k_{1} \leq K^{i_{1}}, \ldots, 0 \leq k_{l} \leq K^{i_{l}}, m=0,-1, a \in A, n \in \alpha+\mathbb{Z}, 0 \leq k \leq K, v \in V^{[\alpha]}, \alpha \in P_{V}$ such that

$$
\Re\left(\operatorname{wt} \phi^{i_{1}}-n_{1}-1+\cdots+\phi^{i_{l}}-n_{l}-1-m+\operatorname{wt} w^{a}-n-1+\operatorname{wt} v\right)<B .
$$

Consider the quotient $U\left(\hat{V}_{\phi}^{[g]}\right)$-module $\widetilde{M}^{[g]} / J_{B}\left(\widetilde{M}^{[g]}\right)$. Since $J_{B}\left(\widetilde{M}^{[g]}\right)$ is spanned by homogeneous elements, $\widetilde{M}^{[g]} / J_{B}\left(\widetilde{M}^{[g]}\right)$ is also graded. In addition, $\widetilde{M}^{[g]} / J_{B}\left(\widetilde{M}^{[g]}\right)$ is lower bounded with respect to the weight grading with a lower bound $B$. We shall still use the same notations to denote the elements of this quotient and operators on this quotient. Then in this quotient $\left(\psi_{\widetilde{M}[g]}^{a}\right)_{n, 0} \mathbf{1}=0$ for $a \in A$, and $n \notin-\mathbb{N}-1$. 
For $i \in I, a \in A, n \in \mathbb{C}$ and $k \in \mathbb{N}$, by (15.2)

$$
\mathrm{wt}\left(\left(\phi_{\widetilde{M}^{[g]}}^{i}\right)_{n, k}\left(\psi_{\widetilde{M}^{[g]}}^{a}\right)_{-1,0} \mathbf{1}\right)=\mathrm{wt} \phi^{i}-n-1+\mathrm{wt} w^{a} .
$$

Since $B$ is a lower bound of the real parts of the weights of $\widetilde{M}^{[g]} / J_{B}\left(\widetilde{M}^{[g]}\right)$ and

$$
\phi_{\widetilde{M}^{[g]}}^{i}(x) \in x^{-\alpha^{i}}\left(\widetilde{M}^{[g]} / J_{B}\left(\widetilde{M}^{[g]}\right)\right)\left[\left[x, x^{-1}\right]\right],
$$

we have

$$
\left(\phi_{\widetilde{M}^{[g]}}^{i}\right)_{n, k}\left(\psi_{\widetilde{M}^{[g]}}^{a}\right)_{-1,0} \mathbf{1}=0
$$

when wt $\left(\phi_{\widetilde{M}^{[g]}}^{i}\right)_{n, k} w^{a}=\alpha^{i}+m$ where $m \in \mathbb{Z}$ and $m>$ wt $\phi^{i}-1+\Re\left(\right.$ wt $\left.w^{a}\right)-B-\Re\left(\alpha^{i}\right)$. For $i \in I$ and $a \in A$, let $M_{i, a} \in \mathbb{Z}_{+}$be the smallest of $m \in \mathbb{Z}$ such that $m>$ wt $\phi^{i}-1+$ $\Re\left(\right.$ wt $\left.w^{a}\right)-B-\Re\left(\alpha^{i}\right)$. Then

$$
x^{\alpha^{i}+M_{i, a}} \phi_{\widetilde{M}^{[g]}}^{i}(x)\left(\psi_{\widetilde{M}^{[g]}}^{a}\right)_{-1,0} \mathbf{1}
$$

is a powers series in $x$ with polynomials in $\log x$ as coefficients. Also for $a \in A$ and $v \in V$, $\psi_{\widetilde{M}^{[g]}}^{a}(x) v$ has only finitely many terms with negative real parts of powers of $x$. In particular, for $i \in I, a \in A$ and $v \in V^{[\alpha]}$,

$$
\begin{aligned}
& \left(x_{1}-x_{2}\right)^{\alpha^{i}+M_{i, a}}\left(x_{1}-x_{2}\right)^{\mathcal{N}_{g}} \phi_{\widetilde{M}^{[g]}}^{i}\left(x_{1}\right)\left(x_{1}-x_{2}\right)^{-\mathcal{N}_{g}} \psi_{\widetilde{M}^{[g]}}^{a}\left(x_{2}\right) v \\
& \quad-(-1)^{|u||w|}\left(-x_{2}+x_{1}\right)^{\alpha^{i}+M_{i, a}} \psi_{\widetilde{M}^{[g]}}^{a}\left(x_{2}\right)\left(-x_{2}+x_{1}\right)^{\mathcal{N}_{g}} \phi^{i}\left(x_{1}\right)\left(-x_{2}+x_{1}\right)^{-\mathcal{N}_{g}} v
\end{aligned}
$$

in $x_{1}$ and $x_{2}$ are well defined as a formal series with coefficients in $\widetilde{M}^{[g]} / J_{B}\left(\widetilde{M}^{[g]}\right)$. Let $J\left(\widetilde{M}^{[g]} / J_{B}\left(\widetilde{M}^{[g]}\right)\right)$ be the $U\left(\hat{V}_{\phi}^{[g]}\right)$-submodule of $\widetilde{M}^{[g]} / J_{B}\left(\widetilde{M}^{[g]}\right)$ generated by the coefficients of the formal series (5.7) for $i \in I, a \in A$ and $v \in V^{[\alpha]}$ and the coefficients of the formal series

$$
\begin{gathered}
L_{\widetilde{M}^{[g]}}(0) \psi_{\widetilde{M}^{[g]}}^{a}(x) v-\psi_{\widetilde{M}^{[g]}}^{a}(x) L_{V}(0) v-x \frac{d}{d x} \psi_{\widetilde{M}^{[g]}}^{a}(x) v-\left(\operatorname{wt} w^{a}\right) \psi_{\widetilde{M}^{[g]}}^{a}(x) v-\psi_{\widetilde{M}_{M}^{[g]}}^{L_{M}(0)_{N} a}(x) v, \\
L_{\widetilde{M}^{[g]}}(-1) \psi_{\widetilde{M}^{[g]}}^{a}(x) v-\psi_{\widetilde{M}^{[g]}}^{a}(x) L_{V}(-1) v-\frac{d}{d x} \psi_{\widetilde{M}^{[g]}}^{a}(x) v
\end{gathered}
$$

for $a \in A$ and $v \in V$. We then have a quotient $U\left(\hat{V}_{\phi}^{[g]}\right)$-module

$$
\widehat{M}_{B}^{[g]}=\left(\widetilde{M}_{\ell}^{[g]} / J_{B}\left(\widetilde{M}^{[g]}\right)\right) / J\left(\widetilde{M}^{[g]} / J_{B}\left(\widetilde{M}^{[g]}\right)\right)
$$

Again, we shall use the same notations for the elements of $\widetilde{M}^{[g]}$ to denote the corresponding elements of $\widehat{M}_{B}^{[g]}$. We shall use $\phi_{\widehat{M}_{B}^{[g]}}^{i}(x), \psi_{\widehat{M}_{B}^{[g]}}^{a}(x), L_{\widehat{M}_{B}^{[g]}}(0)$ and $L_{\widehat{M}_{B}^{[g]}}(-1)$ to denote the series of operators and the operators on $\widehat{M}_{B}^{[g]}$ induced from the corresponding series of operators and operators on $\widetilde{M}^{[g]}$. Since $J\left(\widetilde{M}^{[g]} / J_{B}\left(\widetilde{M}^{[g]}\right)\right)$ is spanned by homogeneous elements, the 
quotient $\widehat{M}_{B}^{[g]}=\left(\widetilde{M}_{\ell}^{[g]} / J_{B}\left(\widetilde{M}^{[g]}\right)\right) / J\left(\widetilde{M}^{[g]} / J_{B}\left(\widetilde{M}^{[g]}\right)\right)$ is also graded and is lower bounded with respect to the weight grading with a lower bound $B$. Moreover, in $\widehat{M}_{B}^{[g]}$, we have

$$
\begin{gathered}
\left(x_{1}-x_{2}\right)^{M_{i j}} \phi_{\widehat{M}_{B}^{[g]}}^{i}\left(x_{1}\right) \phi_{\widehat{M}_{B}^{[g]}}^{j}\left(x_{2}\right)=(-1)^{\left|\phi^{i}\right|\left|\phi^{j}\right|}\left(x_{1}-x_{2}\right)^{M_{i j}} \phi_{\widehat{M}_{B}^{j g]}}^{j}\left(x_{2}\right) \phi_{\widehat{M}_{B}^{[g]}}^{i}\left(x_{1}\right), \\
L_{\widehat{M}_{B}^{[g]}}(0) \phi_{\widehat{M}_{B}^{[g]}}^{i}(x)-\phi_{\widehat{M}_{B}^{[g]}}^{i}(x) L_{\widehat{M}_{B}^{[g]}}(0)=x \frac{d}{d x} \phi_{\widehat{M}_{B}^{[g]}}^{i}(x)+\left(\mathrm{wt} \phi^{i}\right) \phi_{\widehat{M}_{B}^{[g]}}^{i}(x), \\
L_{\widehat{M}_{B}^{[g]}}(-1) \phi_{\widehat{M}_{B}^{[g]}}^{i}(x)-\phi_{\hat{V}_{\phi}^{[g]}}^{i}(x) L_{\widehat{M}_{B}^{[g]}}(-1)=\frac{d}{d x} \phi_{\widehat{M}_{B}^{[g]}}^{i}(x), \\
\left(x_{1}-x_{2}\right)^{\alpha^{i}+M_{i, a}}\left(x_{1}-x_{2}\right)^{\mathcal{N}_{g}} \phi_{\widehat{M}_{B}^{[g]}}^{i}\left(x_{1}\right)\left(x_{1}-x_{2}\right)^{-\mathcal{N}_{g}} \psi_{\widehat{M}_{B}^{[g]}}^{a}\left(x_{2}\right) v \\
=(-1)^{|u||w|}\left(-x_{2}+x_{1}\right)^{\alpha^{i}+M_{i, a}} \psi_{\widehat{M}_{B}^{[g]}}^{a}\left(x_{2}\right)\left(-x_{2}+x_{1}\right)^{\mathcal{N}_{g}} \phi^{i}\left(x_{1}\right)\left(-x_{2}+x_{1}\right)^{-\mathcal{N}_{g}} v, \\
L_{\widehat{M}_{B}^{[g]}}(0) \psi_{\widehat{M}_{B}^{[g]}}^{a}(x) v-\psi_{\widehat{M}_{B}^{[g]}}^{a}(x) L_{V}(0) v=x \frac{d}{d x} \psi_{\widehat{M}_{B}^{[g]}}^{a}(x)+\left(\mathrm{wt} w^{a}\right) \psi_{\widehat{M}_{B}^{[g]}}^{a}(x)+\psi_{\widehat{M}_{B}^{[g]}}^{L_{M}(0)_{N} a}(x), \\
L_{\widehat{M}_{B}^{[g]}}(-1) \psi_{\widehat{M}_{B}^{[g]}}^{a}(x) v-\psi_{\widehat{M}_{B}^{[g]}}^{a}(x) L_{V}(-1) v=\frac{d}{d x} \psi_{\widehat{M}_{B}^{[g]}}^{a}(x) v .
\end{gathered}
$$

By (5.9), (5.10), (15.12) and (15.13), we see that $\widehat{M}_{B}^{[g]}$ is spanned by elements of the form

$$
\left(\phi_{\widehat{M}_{B}^{[g]}}^{i_{1}}\right)_{n_{1}, k_{1}} \cdots\left(\phi_{\widehat{M}_{B}^{[g]}}^{i_{l}}\right)_{n_{l}, k_{l}}\left(\psi_{\widehat{M}_{B}^{[g]}}^{a}\right)_{n, k} v
$$

We now have the following main result giving an explicit construction of lower-bounded generalized $g$-twisted $V$-modules:

Theorem 5.1 The twisted fields $\phi_{\widehat{M}_{B}^{[g]}}^{i}$ for $i \in I$ generate a twisted vertex operator map

$$
Y_{\widehat{M}_{B}^{[g]}}^{g}: V \otimes \widehat{M}_{B}^{[g]} \rightarrow \widehat{M}_{B}^{[g]}\{x\}[\log x]
$$

such that $\left(\widehat{M}_{B}^{[g]}, Y_{\widehat{M}_{B}^{[g]}}^{g}\right)$ is a lower-bounded generalized g-twisted $V$-module. Moreover, this is the unique generalized g-twisted $V$-module structure on $\widehat{M}_{B}^{[g]}$ generated by the coefficients of $\left(\psi_{\widehat{M}_{B}^{[g]}}^{a}\right)(x) v$ for $a \in A$ and $v \in V$ such that $Y_{\widehat{M}_{B}^{[g]}}^{g}\left(\phi_{-1}^{i} \mathbf{1}, z\right)=\phi_{\widehat{M}_{B}^{[g]}}^{i}(z)$ for $i \in I$.

Proof. The space $\widehat{M}_{B}^{[g]}$ is graded by weights, $\mathbb{Z}_{2}$-fermion numbers and is a direct sum of generalized eigenspaces of an action of $g$. By construction, $\left(\widehat{M}_{B}^{[g]}\right)_{[n]}=0$ when $\Re(n)<B$. We already have the linear maps $\phi_{\widehat{M}_{B}^{[g]}}^{i}, \psi_{\widehat{M}_{B}^{[g]}}^{a}, L_{\widehat{M}_{B}^{[g]}}(0)$ and $L_{\widehat{M}_{B}^{[g]}}(-1)$. We need only verify Properties 17 in Assumption 2.3.

By (5.9) and (5.12), Property 1 in Assumption 2.3 holds.

By (5.10) and (5.13), Property 2 in Assumption 2.3 holds.

By the definition of $\widehat{M}_{B}^{[g]}$, Property 3 in Assumption 2.3 holds. 
By the definition of $\widehat{M}_{B}^{[g]}$, Property 4 in Assumption 2.3 holds.

For $i \in I$ and $p \in \mathbb{Z}$, by (5.5) and the definition of the actions of $g, e^{2 \pi i \mathcal{S}_{g}}$ and $\mathcal{N}_{g}$ on $\widehat{M}_{B}^{[g]}$, we have

$$
\begin{aligned}
g \phi_{\widehat{M}_{B}^{i ; p+1}}^{i g)}(z) g^{-1} & =e^{2 \pi i \mathcal{S}_{g}} e^{2 \pi i \mathcal{N}_{g}}\left(\left(e^{-l_{p+1}(z) \mathcal{N}_{g}} \phi_{-1}^{i} \mathbf{1}\right) \otimes \frac{t^{\alpha^{i}}}{e^{\alpha^{i} l_{p+1}(z)}} x^{-1} \delta\left(\frac{t}{z}\right)\right) e^{-2 \pi i \mathcal{N}_{g}} e^{-2 \pi i \mathcal{S}_{g}} \\
& =\left(e^{-\left(l_{p+1}(z)-2 \pi i\right) \mathcal{N}_{g}} e^{2 \pi i \mathcal{S}_{g}} \phi_{-1}^{i} \mathbf{1}\right) \otimes \frac{t^{\alpha^{i}}}{e^{\alpha^{i} l_{p+1}(z)}} z^{-1} \delta\left(\frac{t}{z}\right) \\
& =\left(e^{-\left(l_{p+1}(z)-2 \pi i\right) \mathcal{N}_{g}} \phi_{-1}^{i} \mathbf{1}\right) \otimes \frac{t^{\alpha^{i}}}{e^{\alpha^{i}\left(l_{p+1}(z)-2 \pi i\right)}} z^{-1} \delta\left(\frac{t}{z}\right) \\
& =\left(e^{-l_{p}(z) \mathcal{N}_{g}} a\right) \otimes \frac{t^{\alpha^{i}}}{e^{\alpha^{i} l_{p}(z)}} z^{-1} \delta\left(\frac{t}{z}\right) \\
& =\phi_{\widehat{M}_{B}^{i ; p}}^{[i g}(z) .
\end{aligned}
$$

This is Part (i) of Property 5 in Assumption 2.3. Part (ii) of Property 5 in Assumption 2.3 follows immediately from (5.5), (15.6) and the definition of the action of $\mathcal{N}_{g}$ on $\widehat{M}_{B}^{[g]}$. Part (iii) of Property 5 in Assumption 2.3 follows immediately from the definition of the actions of $e^{2 \pi i \mathcal{S}_{g}}$ and $\mathcal{N}_{g}$ on $\widehat{M}_{B}^{[g]}$ and Assumption 2.1. For $a \in A$ and $n \in-\mathbb{N}-1$, since $w^{a}$ is a generalized eigenvector of $g$ with eigenvalue $e^{2 \pi i \alpha^{a}}$, by the definition of the action of $g$ on $\widehat{M}_{B}^{[g]},\left(\psi_{W}^{a}\right)_{n, 0} \mathbf{1}$ is a generalized eigenvector of $g$ with eigenvalue $e^{2 \pi i \alpha^{a}}$. This is Part (iv) of Property 5 in Assumption 2.3 .

Property 6 in Assumption 2.3 in our case is in fact (5.8).

Property 7 in Assumption 2.3 in our case is in fact (5.11).

Since the space $\widehat{M}_{B}^{[g]}$ equipped with $\phi_{\widehat{M}_{B}^{[g]}}^{i}, \psi_{\widehat{M}_{B}^{[g]}}^{a}, L_{\widehat{M}_{B}^{[g]}}(0)$ and $L_{\widehat{M}_{B}^{[g]}}(-1)$ satisfies Properties 17 7 in Assumption 2.3, by Theorem4.3, we have a unique generalized $g$-twisted $V$-module structure on $\widehat{M}_{B}^{[g]}$ generated by the coefficients of $\left(\psi_{\widehat{M}_{B}^{[g]}}^{a}\right)(x) v$ for $a \in A$ and $v \in V$ such that $Y_{\widehat{M}_{B}^{[g]}}^{g}\left(\phi_{-1}^{i} \mathbf{1}, z\right)=\phi_{\widehat{M}_{B}^{[g]}}^{i}(z)$ for $i \in I$.

Now we prove a universal property of the generalized $g$-twisted $V$-module $\widehat{M}_{B}^{[g]}$ constructed in Theorem 5.1.

Theorem 5.2 Let $\left(W, Y_{W}^{g}\right)$ be a lower-bounded generalized $g$-twisted $V$-module and $M_{0}$ a $\mathbb{Z}_{2}$-graded subspace of $W$ invariant under the actions of $g, \mathcal{S}_{g}, \mathcal{N}_{g}, L_{W}(0), L_{W}(0)_{S}$ and $L_{W}(0)_{N}$. Let $B \in \mathbb{R}$ such that $W_{[n]}=0$ when $\Re(n)<B$. Assume that there is a linear map $f: M \rightarrow M_{0}$ preserving the $\mathbb{Z}_{2}$-fermion number grading and commuting with the actions of $g, \mathcal{S}_{g}, \mathcal{N}_{g}, L_{W}(0)\left(L_{\widehat{M}_{B}^{[g]}}(0)\right), L_{W}(0)_{S}\left(L_{\widehat{M}_{B}^{[g]}}(0)_{S}\right)$ and $L_{W}(0)_{N}\left(L_{\widehat{M}_{B}^{[g]}}(0)_{N}\right)$. Then there exists a unique module map $\tilde{f}: \widehat{M}_{B}^{[g]} \rightarrow W$ such that $\left.\tilde{f}\right|_{M}=f$. If $f$ is surjective and $\left(W, Y_{W}^{g}\right)$ is generated by the coefficients of $\left(Y^{g}\right)_{W V}^{W}\left(w_{0}, x\right) v$ for $w_{0} \in M_{0}$ and $v \in V$, where $\left(Y^{g}\right)_{W V}^{W}$ is the twist vertex operator map obtained from $Y_{W}^{g}$ (see [H6]), then $\tilde{f}$ is surjective. 
Proof. Note that $\widehat{M}_{B}^{[g]}$ is spanned by elements of the form (5.14). We define $\tilde{f}$ by

$$
\begin{aligned}
& \tilde{f}\left(\left(\phi_{\widehat{M}_{B}^{[g]}}^{i_{1}}\right)_{n_{1}, k_{1}} \cdots\left(\phi_{\widehat{M}_{B}^{[g]}}^{i_{l}}\right)_{n_{l}, k_{l}}\left(\psi_{\widehat{M}_{B}^{[g]}}^{a}\right)_{n, k} v\right) \\
& \quad=\left(Y_{W}^{g}\right)_{n_{1}, k_{1}}\left(\phi_{-1}^{i_{1}} \mathbf{1}\right) \cdots\left(Y_{W}^{g}\right)_{n_{l}, k_{l}}\left(\phi_{-1}^{i_{l}} \mathbf{1}\right)\left(\left(Y^{g}\right)_{W V}^{W}\right)_{n, k}\left(f\left(w^{a}\right)\right) v
\end{aligned}
$$

for $i_{1}, \ldots, i_{l} \in I, n_{1} \in \alpha^{i_{1}}+\mathbb{Z}, \ldots, n_{l} \in \alpha^{i_{l}}+\mathbb{Z}, k_{1}, \ldots, k_{l} \in \mathbb{N}, a \in A, \alpha \in P_{V}, n \in \alpha+\mathbb{Z}$, $k \in \mathbb{N}$ and $v \in V^{[\alpha]}$, where for $i \in I, n \in \alpha^{i}+\mathbb{Z}$ and $k \in \mathbb{N},\left(Y_{W}^{g}\right)_{n, k}\left(\phi_{-1}^{i} \mathbf{1}\right)$ is the coefficient of $x^{-n-1}(\log x)^{k}$ in the series $Y_{W}^{g}\left(\phi_{-1}^{i} \mathbf{1}, x\right)$ and for $a \in A, n \in \mathbb{C}$ and $k \in \mathbb{N},\left(\left(Y^{g}\right)_{W V}^{W}\right)_{n, k}\left(f\left(w^{a}\right)\right)$ is the coefficient of $x^{-n-1}(\log x)^{k}$ in the series $\left(Y^{g}\right)_{W V}^{W}\left(f\left(w^{a}\right), x\right)$.

We first need to show that $\tilde{f}$ is well defined. By the construction of $\widehat{M}_{B}^{[g]}$, we see that the only relations among elements of the form $\left(\phi_{\widehat{M}_{B}^{[g]}}^{i_{1}}\right)_{n_{1}, k_{1}} \cdots\left(\phi_{\widehat{M}_{B}^{[g]}}^{i_{l}}\right)_{n_{l}, k_{l}}\left(\psi_{\widehat{M}_{B}^{[g]}}^{a}\right)_{n, k} v$ are the following: $\left(\psi_{\widehat{M}_{B}^{[g]}}^{a}\right)_{n, 0} \mathbf{1}=0$ for $a \in A, n \in \mathbb{N}-1 ;\left(\phi_{\widehat{M}_{B}^{[g]}}^{i_{1}}\right)_{n_{1}, k_{1}} \cdots\left(\phi_{\widehat{M}_{B}^{[g]}}^{i_{l}}\right)_{n_{l}, k_{l}}\left(\psi_{\widehat{M}_{B}^{[g]}}^{a}\right)_{n, k} v=0$ when the real part of its weight is less than $B$; the coefficients of (5.8) $-(5.13)$; the relations among $v \in V$. These relations also hold for elements of the form

$$
\left(Y_{W}^{g}\right)_{n_{1}, k_{1}}\left(\phi_{-1}^{i_{1}} \mathbf{1}\right) \cdots\left(Y_{W}^{g}\right)_{n_{l}, k_{l}}\left(\phi_{-1}^{i_{l}} \mathbf{1}\right)\left(\left(Y^{g}\right)_{W V}^{W}\right)_{n, k}\left(f\left(w^{a}\right)\right) v
$$

of $W$ because $W$ is a lower-bounded generalized $g$-twisted $V$-module such that $W_{[n]}=0$ when $\Re(n)<B$, because the choices of $M_{i, j}$ for $i, j \in I$ and $M_{i, a}$ for $i \in I$ and $a \in A$ depend only on $\phi^{i}$ and $\phi^{j}$ and on $\phi^{i}$, wt $w^{a}=\operatorname{wt}\left(\psi_{\widehat{M}_{B}^{[g]}}^{a}\right)_{-1,0} \mathbf{1}$ and $B$, respectively, and because $f$ commutes with all the operators on $M$ and $M_{0}$. Thus $\tilde{f}$ is indeed well defined.

By the definition of $\tilde{f}$, it is a module map and $\left.\tilde{f}\right|_{M}=f$. Since $\widehat{M}_{B}^{[g]}$ is determined uniquely by $M, \tilde{f}$ is unique.

If $f$ is surjective and $\left(W, Y_{W}^{g}\right)$ is generated by the coefficients of $\left(Y^{g}\right)_{W V}^{W}\left(w_{0}, x\right) v$ for $w_{0} \in M_{0}$ and $v \in V$, then $\left(W, Y_{W}^{g}\right)$ is in fact generated by $\left(Y^{g}\right)_{W V}^{W}(f(w), x) v$ for $w \in M$ and $v \in V$. Since $\tilde{f}$ is a module map, we obtain $\tilde{f}\left(\widehat{M}_{B}^{[g]}\right)=W$.

Finally we have the following immediate consequence:

Corollary 5.3 Let $\left(W, Y_{W}^{g}\right)$ be a lower-bounded generalized $g$-twisted $V$-module generated by the coefficients of $\left(Y^{g}\right)_{W V}^{W}(w, x) v$ for $w \in M$, where $\left(Y^{g}\right)_{W V}^{W}$ is the twist vertex operator map obtained from $Y_{W}^{g}$ (see [H6]) and $M$ is a $\mathbb{Z}_{2}$-graded subspace of $W$ invariant under the actions of $g, \mathcal{S}_{g}, \mathcal{N}_{g}, L_{W}(0), L_{W}(0)_{S}$ and $L_{W}(0)_{N}$. Let $B \in \mathbb{R}$ such that $W_{[n]}=0$ when $\Re(n)<B$. Then there is a generalized g-twisted $V$-submodule $J$ of $\widehat{M}_{B}^{[g]}$ such that $W$ is equivalent as a lower-bounded generalized g-twisted $V$-module to the quotient module $\widehat{M}_{B}^{[g]} / J$.

\section{References}

[B] B. Bakalov, Twisted logarithmic modules of vertex algebras, Comm. Math. Phys. 345 (2015), 355-383. 
[BDM $] \quad$ K. Barron, C. Dong and G. Mason, Twisted sectors for tensor products vertex operator algebras associated to permutation groups, Commun. Math. Phys., 227 (2002), 349-384.

[BHL] K. Barron, Y.-Z. Huang and J. Lepowsky, An equivalence of two constructions of permutation-twisted modules for lattice vertex operator algebras, Jour. Pure Appl. Alg. 210 (2007), 797-826.

[D] C. Dong, Twisted modules for vertex algebras associated with even lattice, J. of Algebra 165 (1994), 91-112.

[DL] C. Dong and J. Lepowsky, The algebraic structure of relative twisted vertex operators, J. Pure Appl. Algebra 110 (1996), 259-295.

[DonLM1] C. Dong, H. Li and G. Mason, Twisted representations of vertex operator algebras, Math. Ann. 310 (1998), 571-600.

[DonLM2] C. Dong, H. Li and G. Mason, Modular invariance of trace functions in orbifold theory and generalized moonshine, Commun. Math. Phys., 214 (2000), 1-56.

[DoyLM1] B. Doyon, J. Lepowsky and A. Milas, Twisted modules for vertex operator algebras and Bernoulli polynomials, Int. Math. Res. Not. 44 (2003), 2391-2408.

[DoyLM2] B. Doyon, J. Lepowsky and A. Milas, Twisted vertex operators and Bernoulli polynomials, Commun. in Contemporary Math. 8 (2006), 247-307.

[FLM1] I. Frenkel, J. Lepowsky and A. Meurman, A natural representation of the FischerGriess Monster with the modular function $J$ as character, Proc. Natl. Acad. Sci. USA 81 (1984), 3256-3260.

[FLM2] I. Frenkel, J. Lepowsky and A. Meurman, Vertex operator calculus, in: Mathematical Aspects of String Theory, Proc. 1986 Conference, San Diego, ed. by S.-T. Yau, World Scientific, Singapore, 1987, 150-188.

[FLM3] I. Frenkel, J. Lepowsky and A. Meurman, Vertex Operator Algebras and the Monster, Pure and Applied Math., Vol. 134, Academic Press, 1988.

[H1] Y.-Z. Huang, Generalized twisted modules associated to general automorphisms of a vertex operator algebra, Comm. Math. Phys. 298 (2010), 265-292.

[H2] Y.-Z. Huang, Two constructions of grading-restricted vertex (super)algebras, $J$. Pure Appl. Alg. 220 (2016), 3628-3649.

[H3] Y.-Z. Huang, Some open probelms in mathematical two-dimensional conformal field theory, in: Proceedings of the Conference on Lie Algebras, Vertex Operator Algebras, and Related Topics, held at University of Notre Dame, Notre Dame, Indiana, August 14-18, 2015, ed. K. Barron, E. Jurisich, H. Li, A. Milas, K. C. 
Misra, Contemp. Math, Vol. 695, American Mathematical Society, Providence, RI, 2017, 123-138.

[H4] Y.-Z. Huang, On the applicability of logarithmic tensor category theory, to appear; arXiv:1702.00133.

[H5] Y.-Z. Huang, Intertwining operators among twisted modules associated to notnecessarily-commuting automorphisms, J. Alg. 493 (2018), 346-380.

[H6] Y.-Z. Huang, Twist vertex operators for twisted modules, J. Alg. 539 (2019), $53-83$.

[HLZ] Y.-Z. Huang, J, Lepowsky and L. Zhang, Logarithmic tensor category theory for generalized modules for a conformal vertex algebra, V: Convergence condition for intertwining maps and the corresponding compatibility condition, arXiv: 1012.4199.

[HY] Yi-Zhi Huang and Jinwei Yang, Associative algebras for (logarithmic) twisted modules for a vertex operator algebra, Trans. Amer. Math. Soc. 371 (2019), $3747-3786$.

[Le1] J. Lepowsky, Calculus of twisted vertex operators, Proc. Nat. Acad. Sci. USA 82 (1985), 8295-8299.

[Le2] J. Lepowsky, Perspectives on vertex operators and the Monster, in: Proc. 1987 Symposium on the Mathematical Heritage of Hermann Weyl, Duke Univ., Proc. Symp. Pure. Math., American Math. Soc. 48 (1988), 181-197.

[Li] H. Li, Local systems of twisted vertex operators, vertex operator superalgebras and twisted modules, in: Moonshine, the Monster, and related topics Mount Holyoke, 1994, ed. C. Dong and G. Mason, Contemporary Math., Vol. 193, Amer. Math. Soc., Providence, 1996, 203-236.

[Y] J. Yang, Twisted representations of vertex operator algebras associated to affine Lie algebras, J. Alg. 484 (2017), 88-108.

Department of Mathematics, Rutgers University, 110 Frelinghuysen Rd., PiscatAWAY, NJ 08854-8019

E-mail address: yzhuang@math.rutgers.edu 